Network Working Group

Request for Comments: 1002

March, 1987

\author{
PROTOCOL STANDARD FOR A NEtBIOS SERVICE \\ ON A TCP/UDP TRANSPORT: \\ DETAILED SPECIFICATIONS
}

\begin{abstract}
This RFC defines a proposed standard protocol to support NetBIOS services in a TCP/IP environment. Both local network and internet operation are supported. Various node types are defined to accommodate local and internet topologies and to allow operation with or without the use of IP broadcast.

This RFC gives the detailed specifications of the NetBIOS-over-TCP packets, protocols, and defined constants and variables. A more general overview is found in a companion RFC, "Protocol standard For a NetBIOS Service on a TCP/UDP Transport: Concepts and Methods".
\end{abstract}


TABLE OF CONTENTS

1. STATUS OF THIS MEMO

2. ACKNOWLEDGEMENTS 4

3. INTRODUCTION

4. PACKET DESCRIPTIONS

4.1 NAME FORMAT $r$

4.2 NAME SERVICE PACKETS 27

4.2.1 GENERAL FORMAT OF NAME SERVICE PACKETS

4.2.1.1 HEADER 8

$\begin{array}{lll}4.2 .1 .2 & \text { QUESTION SECTION } & 10\end{array}$

4.2.1.3 RESOURCE RECORD 11

4.2.2 NAME REGISTRATION REQUEST 13

4.2.3 NAME OVERWRITE REQUEST \& DEMAND 14

4.2.4 NAME REFRESH REQUEST 15

4.2.5 POSITIVE NAME REGISTRATION RESPONSE 16

4.2.6 NEGATIVE NAME REGISTRATION RESPONSE 16

4.2.7 END-NODE CHALLENGE REGISTRATION RESPONSE 17

4.2.8 NAME CONFLICT DEMAND 18

4.2.9 NAME RELEASE REQUEST \& DEMAND 19

4.2.10 POSITIVE NAME RELEASE RESPONSE 20

4.2.11 NEGATIVE NAME RELEASE RESPONSE 20

$\begin{array}{lll}\text { 4.2.12 NAME QUERY REQUEST } & 21\end{array}$

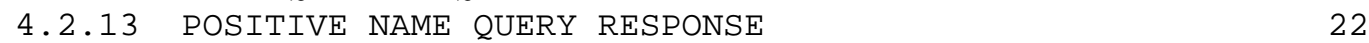

4.2.14 NEGATIVE NAME QUERY RESPONSE 23

4.2.15 REDIRECT NAME QUERY RESPONSE 24

4.2.16 WAIT FOR ACKNOWLEDGEMENT (WACK) RESPONSE

$\begin{array}{lll}4.2 .17 & \text { NODE STATUS REQUEST } & 26\end{array}$

$\begin{array}{ll}\text { 4.2.18 NODE STATUS RESPONSE } & 27\end{array}$

4.3 SESSION SERVICE PACKETS 29

4.3.1 GENERAL FORMAT OF SESSION PACKETS

$\begin{array}{lll}4.3 .2 & \text { SESSION REQUEST PACKET } & 30\end{array}$

4.3.3 POSITIVE SESSION RESPONSE PACKET

4.3.4 NEGATIVE SESSION RESPONSE PACKET

4.3.5 SESSION RETARGET RESPONSE PACKET $\quad 31$

4.3.6 SESSION MESSAGE PACKET

4.3.7 SESSION KEEP ALIVE PACKET

4.4 DATAGRAM SERVICE PACKETS $\quad 32$

4.4.1 NetBIOS DATAGRAM HEADER $\quad 32$

4.4.2 DIRECT_UNIQUE, DIRECT_GROUP, \& BROADCAST DATAGRAM 33

4.4.3 DATAGRAM ERROR PACKET $\quad 34$

4.4.4 DATAGRAM QUERY REQUEST 234

4.4.5 DATAGRAM POSITIVE AND NEGATIVE QUERY RESPONSE

5. PROTOCOL DESCRIPTIONS 35

5.1 NAME SERVICE PROTOCOLS $\quad 35$

$\begin{array}{lll}5.1 .1 & \text { B-NODE ACTIVITY } & 35\end{array}$ 


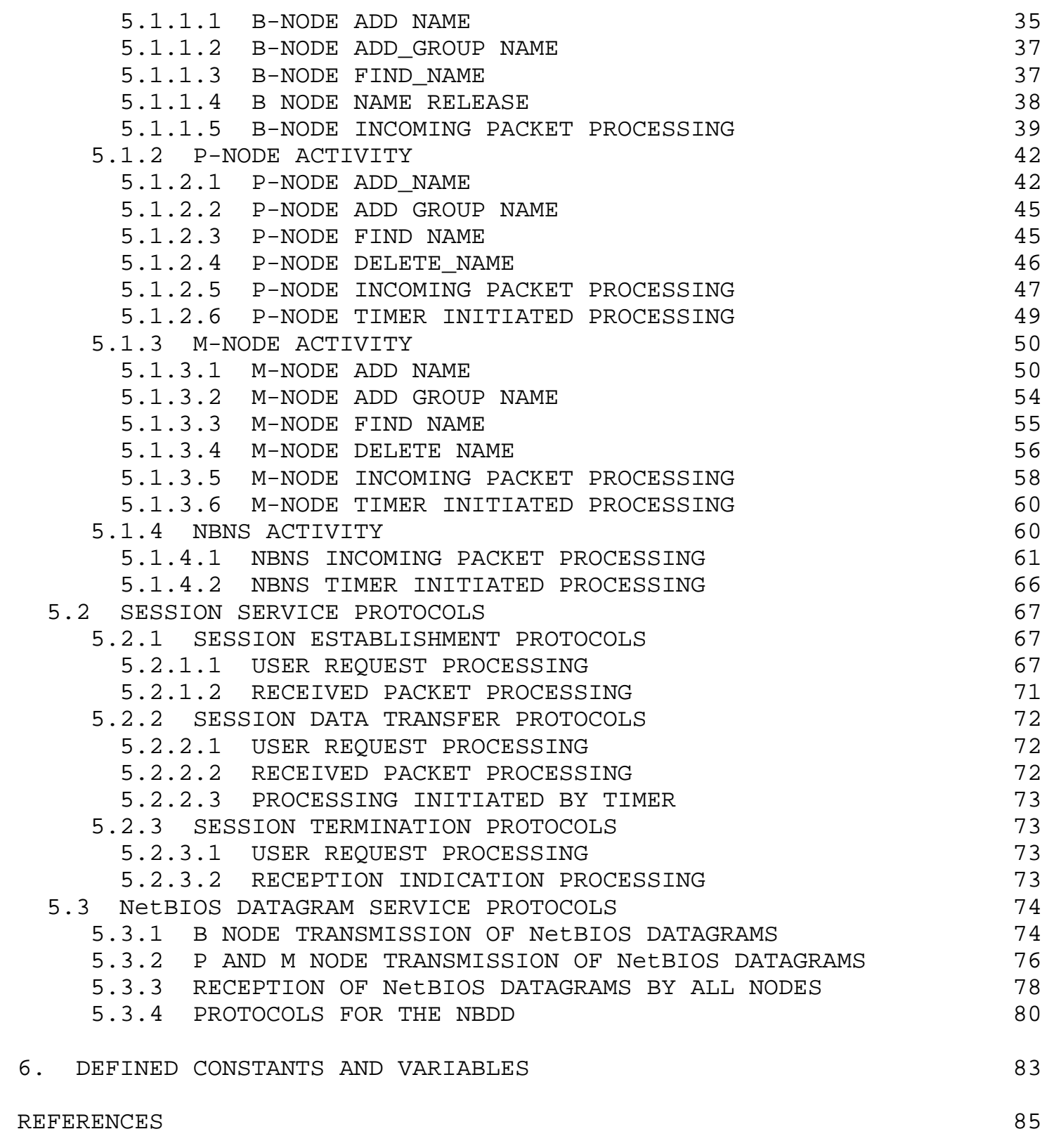




\section{PROTOCOL STANDARD FOR A NetBIOS SERVICE \\ ON A TCP/UDP TRANSPORT: \\ DETAILED SPECIFICATIONS}

1. STATUS OF THIS MEMO

This RFC specifies a proposed standard for the DARPA Internet community. Since this topic is new to the Internet community, discussions and suggestions are specifically requested.

Please send written comments to:

Karl Auerbach

Epilogue Technology Corporation

P.O. Box 5432

Redwood City, CA 94063

Please send online comments to:

Avnish Aggarwal

Internet: mtxinu!excelan!avnish@ucbvax.berkeley.edu

Usenet: ucbvax!mtxinu!excelan!avnish

Distribution of this memorandum is unlimited.

\section{ACKNOWLEDGEMENTS}

This RFC has been developed under the auspices of the Internet Activities Board.

The following individuals have contributed to the development of this RFC:

\begin{tabular}{|c|c|}
\hline Avnish Aggarwal & Lorenzo Aguilar \\
\hline Geoffrey Arnold & K. Ramesh Babu \\
\hline Keith Ball & Amatzia Ben-Artzi \\
\hline Richard Cherry & David Crocker \\
\hline Greg Ennis & Steve Holmgren \\
\hline David Kaufman & Lee LaBarre \\
\hline Dan Lynch & Gaylord Miyata \\
\hline Steve Thomas & Ishan Wu \\
\hline $\begin{array}{l}\text { The system proposed by } \\
\text { Netbios-over-TCP impler } \\
\text { incorporates consideral } \\
\text { implementations. Spec } \\
\text { organizations which hat }\end{array}$ & $\begin{array}{l}\text { this RFC does not reflect any existing } \\
\text { mentation. However, the design } \\
\text { ible knowledge obtained from prior } \\
\text { ial thanks goes to the following } \\
\text { ve provided this invaluable information: }\end{array}$ \\
\hline Excelan & Ungermann-Bass \\
\hline
\end{tabular}




\section{INTRODUCTION}

This RFC contains the detailed packet formats and protocol specifications for NetBIOS-over-TCP. This RFC is a companion to RFC 1001, "Protocol Standard For a NetBIOS Service on a TCP/UDP Transport: Concepts and Methods" [1].

\section{PACKET DESCRIPTIONS}

Bit and byte ordering are defined by the most recent version of "Assigned Numbers" [2].

\subsection{NAME FORMAT}

The NetBIOS name representation in all NetBIOS packets (for NAME, SESSION, and DATAGRAM services) is defined in the Domain Name Service RFC 883[3] as "compressed" name messages. This format is called "second-level encoding" in the section entitled "Representation of NetBIOS Names" in the Concepts and Methods document.

For ease of description, the first two paragraphs from page 31, the section titled "Domain name representation and compression", of $\mathrm{RFC} 883$ are replicated here:

Domain names messages are expressed in terms of a sequence of labels. Each label is represented as a one octet length field followed by that number of octets. Since every domain name ends with the null label of the root, a compressed domain name is terminated by a length byte of zero. The high order two bits of the length field must be zero, and the remaining six bits of the length field limit the label to 63 octets or less.

To simplify implementations, the total length of label octets and label length octets that make up a domain name is restricted to 255 octets or less.

The following is the uncompressed representation of the NetBIOS name "FRED ", which is the 4 ASCII characters, F, R, E, D, followed by 12 space characters $(0 \times 20)$. This name has the SCOPE_ID: "NETBIOS.COM"

$$
\text { EGFCEFEECACACACACACACACACACACACA.NETBIOS.COM }
$$

This uncompressed representation of names is called "first-level encoding" in the section entitled "Representation of NetBIOS Names" in the Concepts and Methods document.

The following is a pictographic representation of the compressed representation of the previous uncompressed Domain Name representation. 
$\begin{array}{llllllllllllllllllllll}1 & 1 & 1 & 1 & 1 & 1 & 1 & 1 & 1 & 1 & 2 & 2 & 2 & 2 & 2 & 2 & 2 & 2 & 2 & 2 & 3 & 3\end{array}$ $\begin{array}{llllllllllllllllllllllllllllllll}0 & 1 & 2 & 3 & 4 & 5 & 6 & 7 & 8 & 9 & 0 & 1 & 2 & 3 & 4 & 5 & 6 & 7 & 8 & 9 & 0 & 1 & 2 & 3 & 4 & 5 & 6 & 7 & 8 & 9 & 0 & 1\end{array}$

\begin{tabular}{|c|c|c|c|}
\hline $0 \times 20$ & $\mathrm{E}(0 \times 45)$ & $\mathrm{G}(0 \times 47)$ & $\mathrm{F}(0 \times 46)$ \\
\hline \multicolumn{4}{|c|}{+-+-+-+-+-+-+-+-+-+-+-+-+-+-+-+-+-+-+-+-+-+-+-+-+-+-+-+-+-+-+-+} \\
\hline C $(0 \times 43)$ & $\mathrm{E}(0 \times 45)$ & $\mathrm{F}(0 \times 46)$ & $\mathrm{E}(0 \times 45)$ \\
\hline \multicolumn{4}{|c|}{-+-+-+-+-+-+-+-+-+-+-+-+-+-+-+-+-+-+-+-+-+-+-+-+-+-+-+-+-+-+-+-+} \\
\hline$E(0 \times 45)$ & C $(0 \times 43)$ & A $(0 \times 41)$ & C $(0 \times 43)$ \\
\hline \multicolumn{4}{|c|}{+-+-+-+-+-+-+-+-+-+-+-+-+-+-+-+-+-+-+-+-+-+-+-+-+-+-+-+-+-+-+-+} \\
\hline A $(0 \times 41)$ & C $(0 \times 43)$ & A $(0 \times 41)$ & C $(0 \times 43)$ \\
\hline \multicolumn{4}{|c|}{-+-+-+-+-+-+-+-+-+-+-+-+-+-+-+-+-+-+-+-+-+-+-+-+-+-+-+-+-+-+-+-+} \\
\hline A $(0 \times 41)$ & C $(0 \times 43)$ & A $(0 \times 41)$ & C $(0 \times 43)$ \\
\hline \multicolumn{4}{|c|}{+-+-+-+-+-+-+-+-+-+-+-+-+-+-+-+-+-+-+-+-+-+-+-+-+-+-+-+-+-+-+-+} \\
\hline A $(0 \times 41)$ & C $(0 \times 43)$ & A $(0 \times 41)$ & C $(0 \times 43)$ \\
\hline \multicolumn{4}{|c|}{+-+-+-+-+-+-+-+-+-+-+-+-+-+-+-+-+-+-+-+-+-+-+-+-+-+-+-+-+-+-+-+} \\
\hline \multirow{2}{*}{\multicolumn{4}{|c|}{\begin{tabular}{c|c|c|c|}
$\mathrm{A}(0 \times 41)$ & $\mathrm{C}(0 \times 43)$ & $\mathrm{A}(0 \times 41)$ & $\mathrm{C}(0 \times 43)$
\end{tabular}}} \\
\hline & & & \\
\hline A $(0 \times 41)$ & C $(0 \times 43)$ & A $(0 \times 41)$ & C $(0 \times 43)$ \\
\hline \multicolumn{4}{|c|}{+-+-+-+-+-+-+-+-+-+-+-+-+-+-+-+-+-+-+-+-+-+-+-+-+-+-+-+-+-+-+-+} \\
\hline A $(0 \times 41)$ & $0 \times 07$ & $\mathrm{~N}(\mathrm{O} \times 4 \mathrm{E})$ & $E(0 \times 45)$ \\
\hline \multicolumn{4}{|c|}{+-+-+-+-+-+-+-+-+-+-+-+-+-+-+-+-+-+-+-+-+-+-+-+-+-+-+-+-+-+-+-+} \\
\hline $\mathrm{T}(0 \times 54)$ & B $(0 \times 42)$ & I $(0 \times 49)$ & $O(0 \times 4 F)$ \\
\hline \multicolumn{4}{|c|}{-+-+-+-+-+-+-+-+-+-+-+-+-+-+-+-+-+-+-+-+-+-+-+-+-+-+-+-+-+-+-+-+} \\
\hline$S(0 \times 53)$ & $0 \times 03$ & C $(0 \times 43)$ & $O(0 \times 4 F)$ \\
\hline & & & \\
\hline x4D) & & & \\
\hline
\end{tabular}

Each section of a domain name is called a label [7 (page 31)]. A label can be a maximum of 63 bytes. The first byte of a label in compressed representation is the number of bytes in the label. For the above example, the first $0 \times 20$ is the number of bytes in the left-most label, EGFCEFEECACACACACACACACACACACACA, of the domain name. The bytes following the label length count are the characters of the label. The following labels are in sequence after the first label, which is the encoded NetBIOS name, until a zero (0x00) length count. The zero length count represents the root label, which is always null.

A label length count is actually a 6-bit field in the label length field. The most significant 2 bits of the field, bits 7 and 6 , are flags allowing an escape from the above compressed representation. If bits 7 and 6 are both set (11), the following 14 bits are an offset pointer into the full message to the actual label string from another domain name that belongs in this name. This label pointer allows for a further compression of a domain name in a packet.

NetBIOS implementations can only use label string pointers in Name Service packets. They cannot be used in Session or Datagram Service packets. 
The other two possible values for bits 7 and 6 (01 and 10) of a label length field are reserved for future use by RFC 883[2 (page 32)].

Note that the first octet of a compressed name must contain one of the following bit patterns. (An "x" indicates a bit whose value may be either 0 or 1.$)$ :

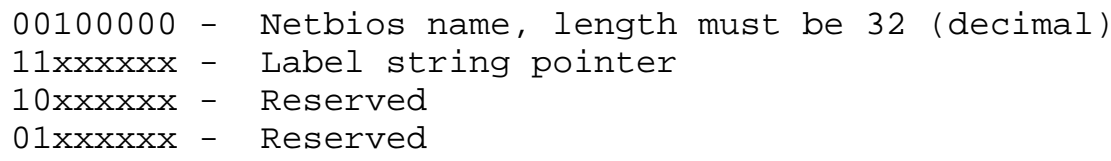

\subsection{NAME SERVICE PACKETS}

\subsubsection{GENERAL FORMAT OF NAME SERVICE PACKETS}

The NetBIos Name Service packets follow the packet structure defined in the Domain Name Service (DNS) RFC 883 [7 (pg 26-31)]. The structures are compatible with the existing DNS packet formats, however, additional types and codes have been added to work with NetBIOS.

If Name Service packets are sent over a TCP connection they are preceded by a 16 bit unsigned integer representing the length of the Name Service packet.

$\begin{array}{llllllllllllllllllllll}1 & 1 & 1 & 1 & 1 & 1 & 1 & 1 & 1 & 1 & 2 & 2 & 2 & 2 & 2 & 2 & 2 & 2 & 2 & 2 & 3 & 3\end{array}$

$\begin{array}{llllllllllllllllllllllllllllllll}0 & 1 & 2 & 3 & 4 & 5 & 6 & 7 & 8 & 9 & 0 & 1 & 2 & 3 & 4 & 5 & 6 & 7 & 8 & 9 & 0 & 1 & 2 & 3 & 4 & 5 & 6 & 7 & 8 & 9 & 0 & 1\end{array}$

$+-+-+-+-+-+-+-+-+-+-+-+-+-+-+-+-+-+-+-+-+-+-+-+-+-+-+-+-+-+-+-+-+$

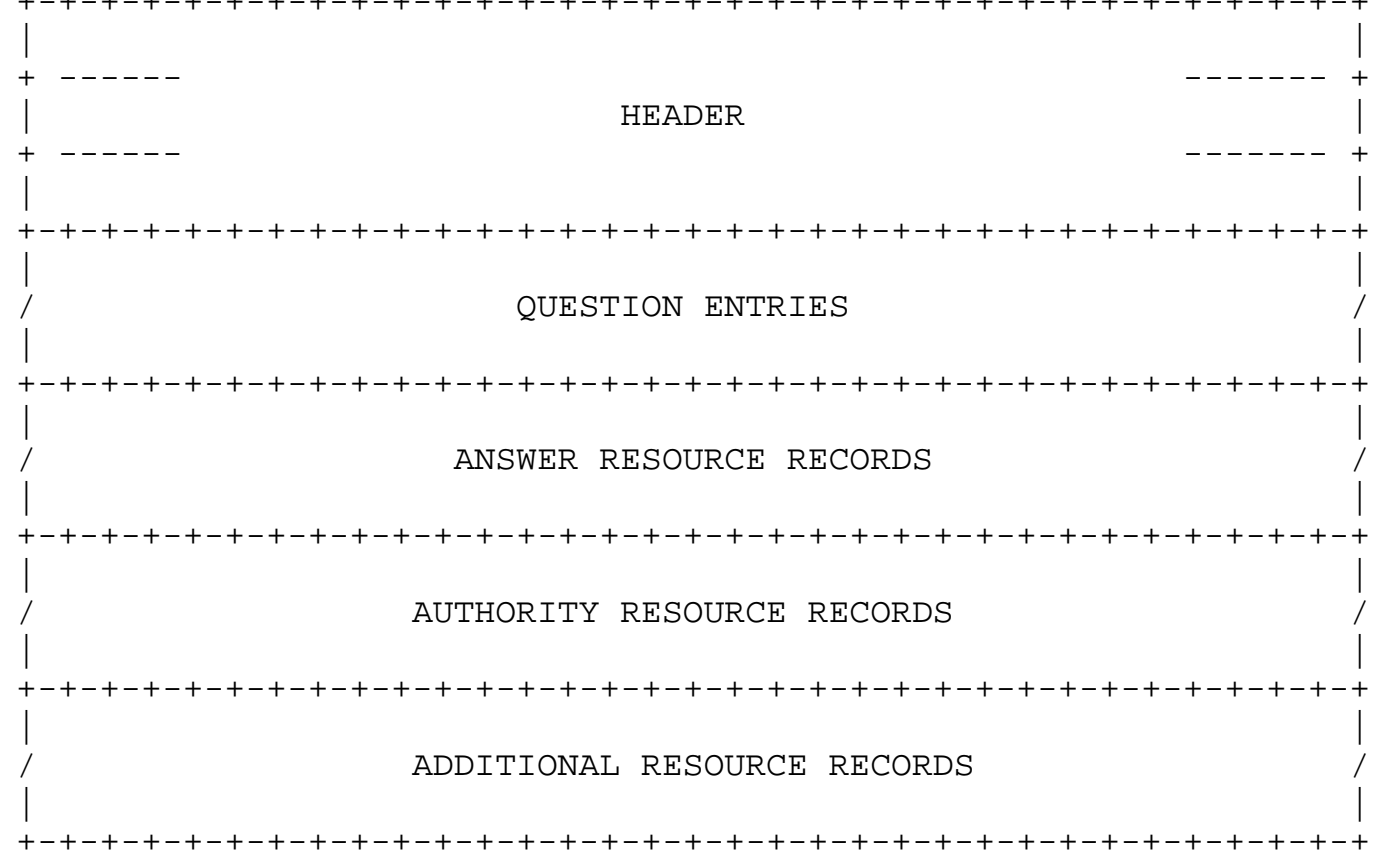




\subsubsection{HEADER}

$\begin{array}{llllllllllllllllllllll}1 & 1 & 1 & 1 & 1 & 1 & 1 & 1 & 1 & 1 & 2 & 2 & 2 & 2 & 2 & 2 & 2 & 2 & 2 & 2 & 3 & 3\end{array}$

$\begin{array}{llllllllllllllllllllllllllllllll}0 & 1 & 2 & 3 & 4 & 5 & 6 & 7 & 8 & 9 & 0 & 1 & 2 & 3 & 4 & 5 & 6 & 7 & 8 & 9 & 0 & 1 & 2 & 3 & 4 & 5 & 6 & 7 & 8 & 9 & 0 & 1\end{array}$

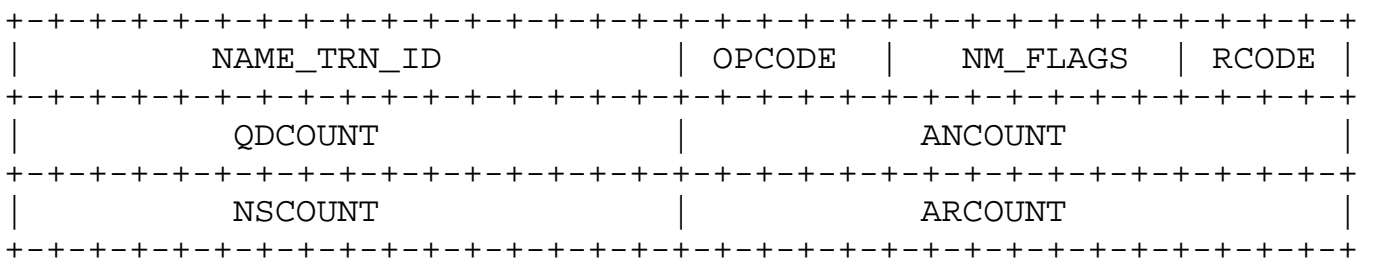

Field Description

NAME_TRN_ID Transaction ID for Name Service Transaction. Requestor places a unique value for each active transaction. Responder puts NAME_TRN_ID value from request packet in response packet.

OPCODE Packet type code, see table below.

NM_FLAGS Flags for operation, see table below.

RCODE Result codes of request. Table of RCODE values for each response packet below.

QDCOUNT

Unsigned 16 bit integer specifying the number of entries in the question section of a Name

Service packet. Always zero (0) for responses. Must be non-zero for all NetBIOS Name requests.

ANCOUNT

Unsigned 16 bit integer specifying the number of resource records in the answer section of a Name Service packet.

NSCOUNT Unsigned 16 bit integer specifying the number of resource records in the authority section of a Name Service packet.

ARCOUNT Unsigned 16 bit integer specifying the number of resource records in the additional records section of a Name Service packet.

The OPCODE field is defined as:

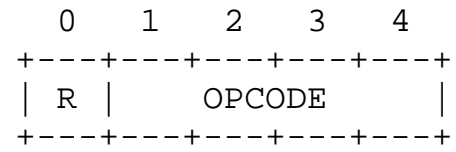




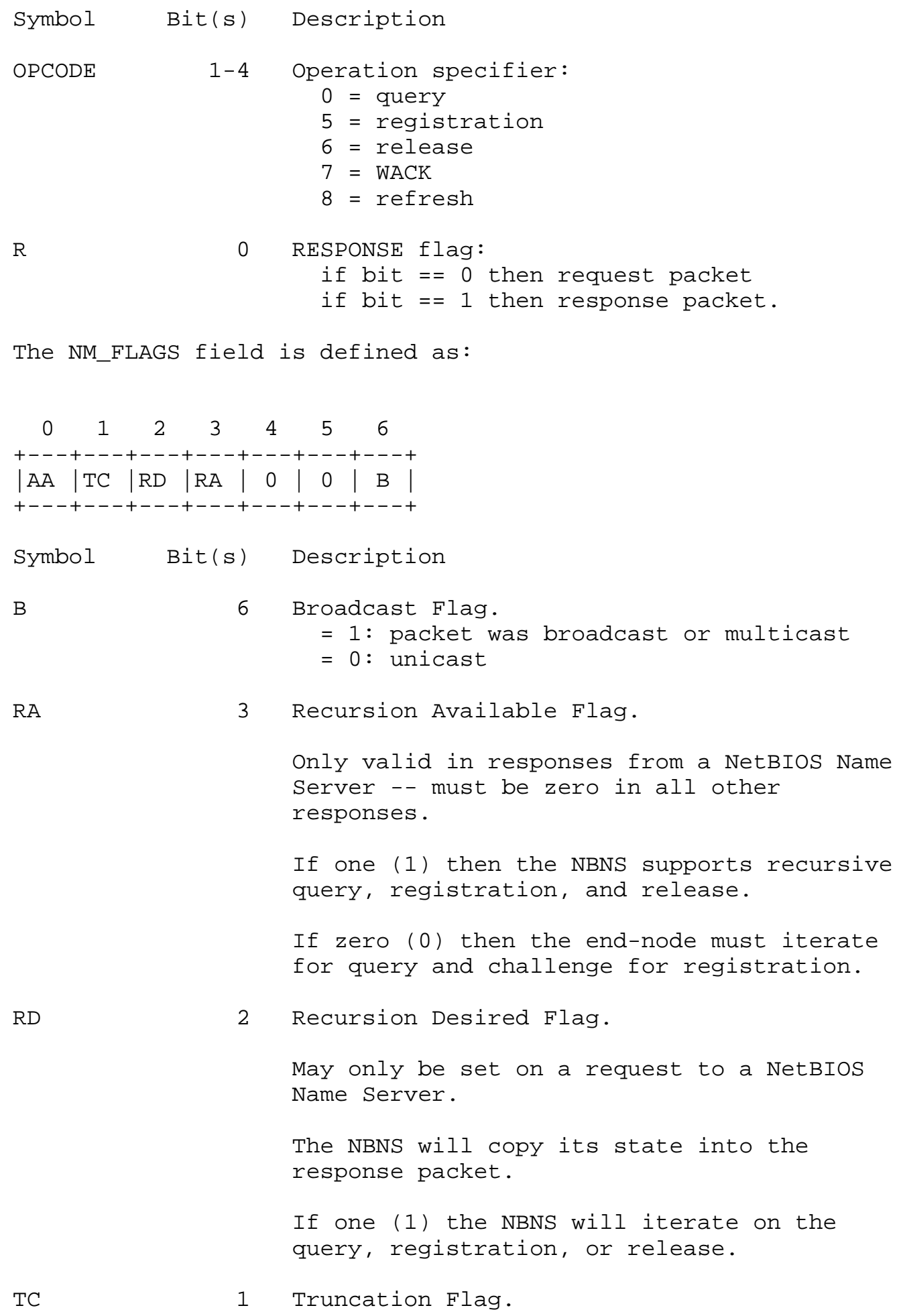


$\mathrm{AA}$
Set if this message was truncated because the datagram carrying it would be greater than 576 bytes in length. Use TCP to get the information from the NetBIOs Name Server.

0 Authoritative Answer flag.

Must be zero (0) if $R$ flag of OPCODE is zero (0).

If $R$ flag is one (1) then if $A A$ is one (1) then the node responding is an authority for the domain name.

End nodes responding to queries always set this bit in responses.

\subsubsection{QUESTION SECTION}

$\begin{array}{llllllllllllllllllllll}1 & 1 & 1 & 1 & 1 & 1 & 1 & 1 & 1 & 1 & 2 & 2 & 2 & 2 & 2 & 2 & 2 & 2 & 2 & 2 & 3 & 3\end{array}$

$\begin{array}{llllllllllllllllllllllllllllllll}0 & 1 & 2 & 3 & 4 & 5 & 6 & 7 & 8 & 9 & 0 & 1 & 2 & 3 & 4 & 5 & 6 & 7 & 8 & 9 & 0 & 1 & 2 & 3 & 4 & 5 & 6 & 7 & 8 & 9 & 0 & 1\end{array}$

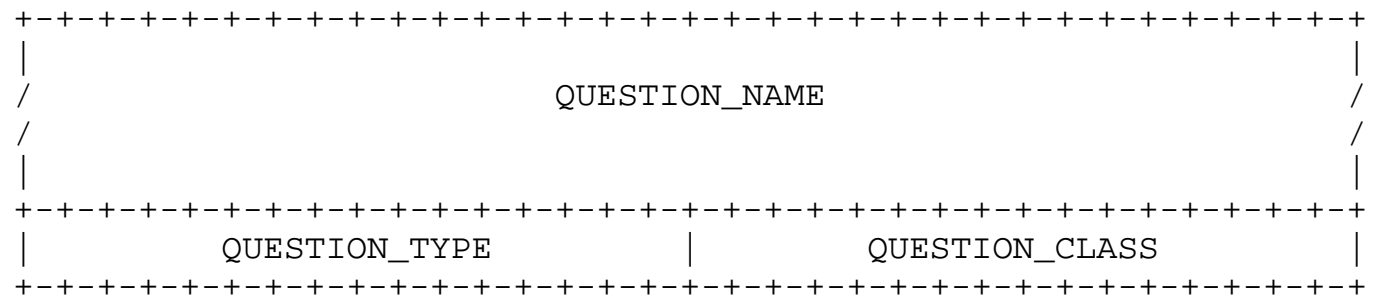

Field Description

QUESTION_NAME The compressed name representation of the NetBIOS name for the request.

QUESTION_TYPE The type of request. The values for this field are specified for each request.

QUESTION_CLASS The class of the request. The values for this field are specified for each request.

QUESTION_TYPE is defined as:

Symbol Value Description:

NB 0x0020 NetBIOS general Name Service Resource Record NBSTAT 0x0021 NetBIOS NODE STATUS Resource Record (See NODE STATUS REQUEST)

QUESTION_CLASS is defined as: 
Symbol Value Description:

IN 0x0001 Internet class

\subsubsection{RESOURCE RECORD}

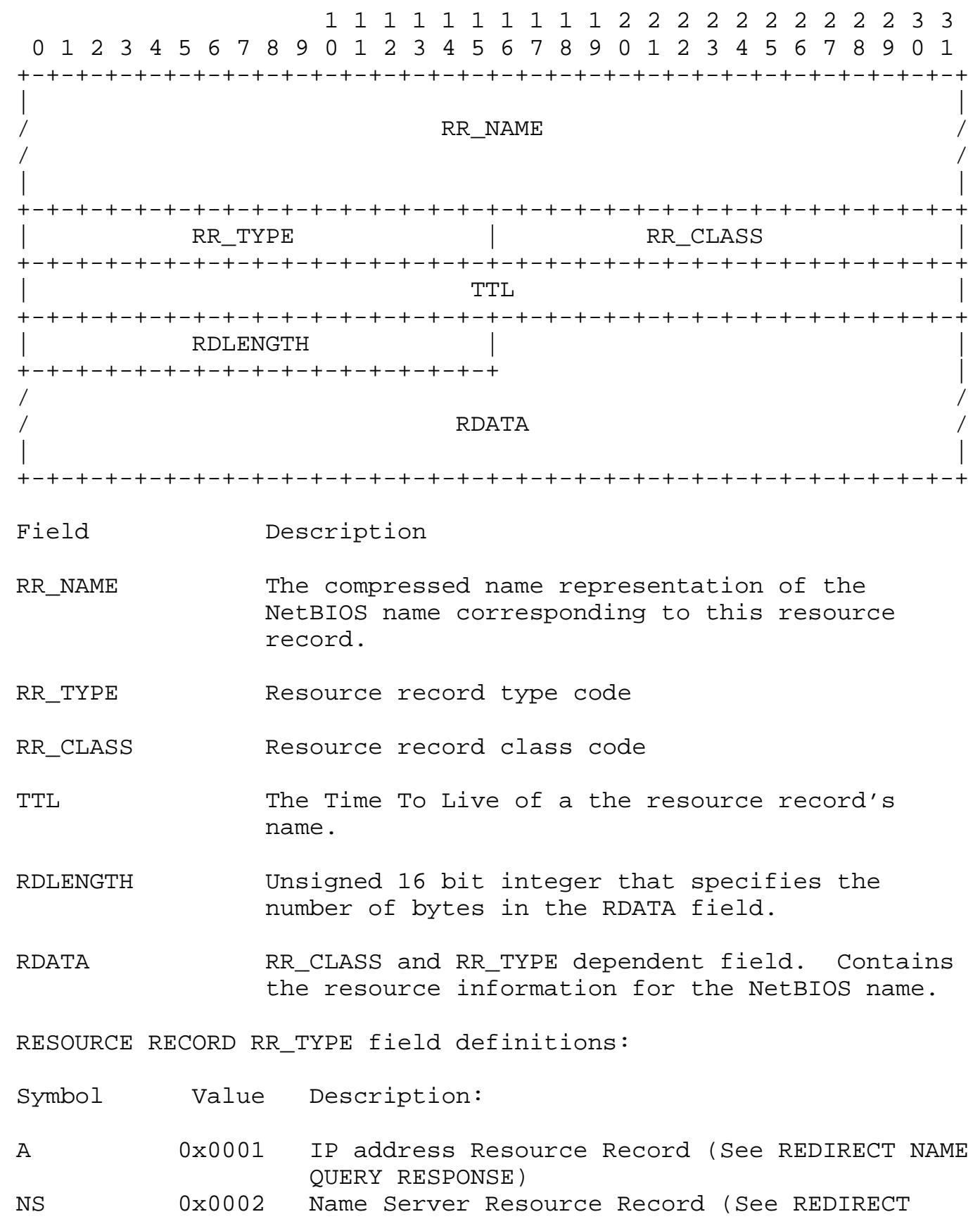




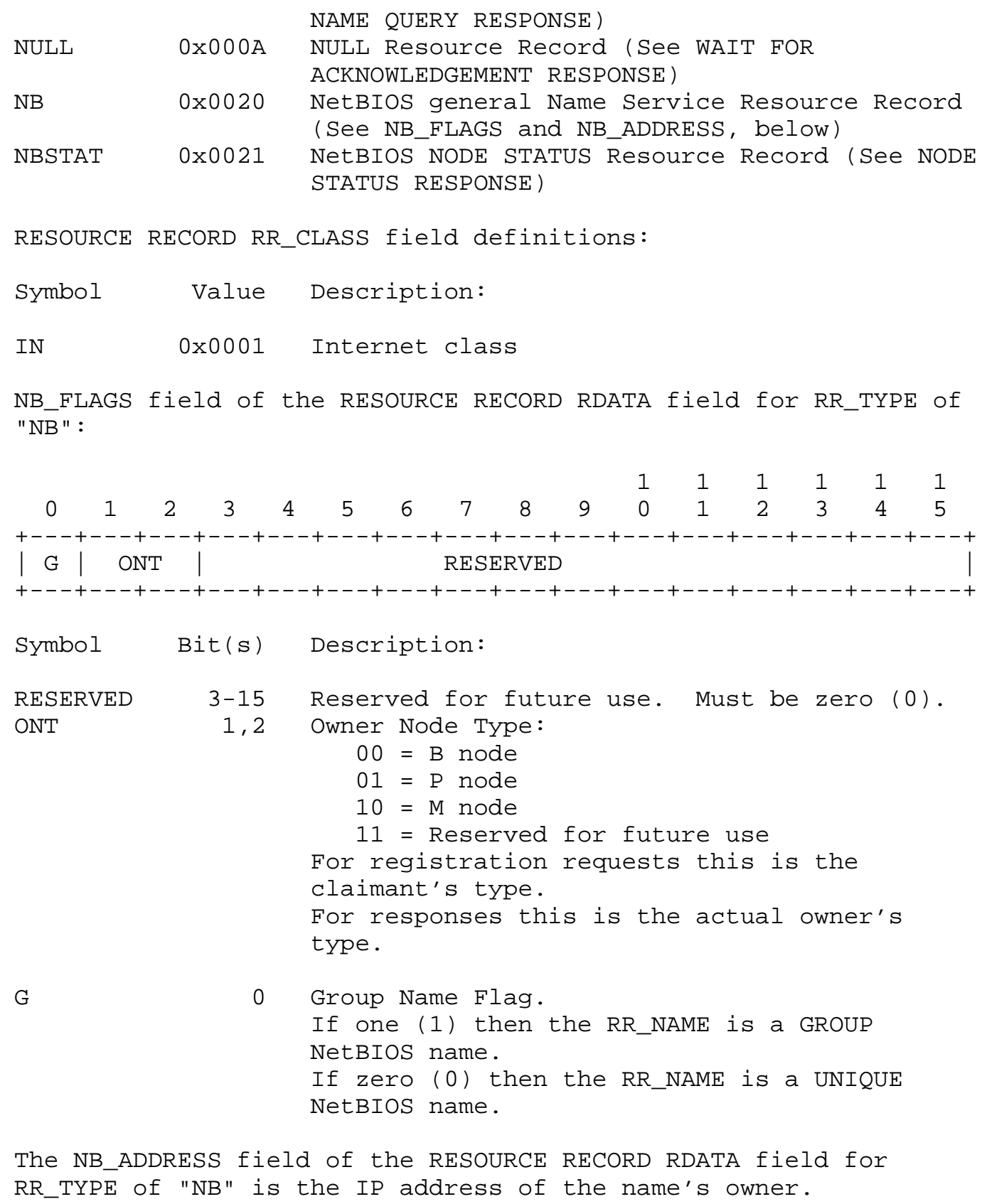

The NB_ADDRESS field of the RESOURCE RECORD RDATA field for RR_TYPE of "NB" is the IP address of the name's owner. 


\subsubsection{NAME REGISTRATION REQUEST}

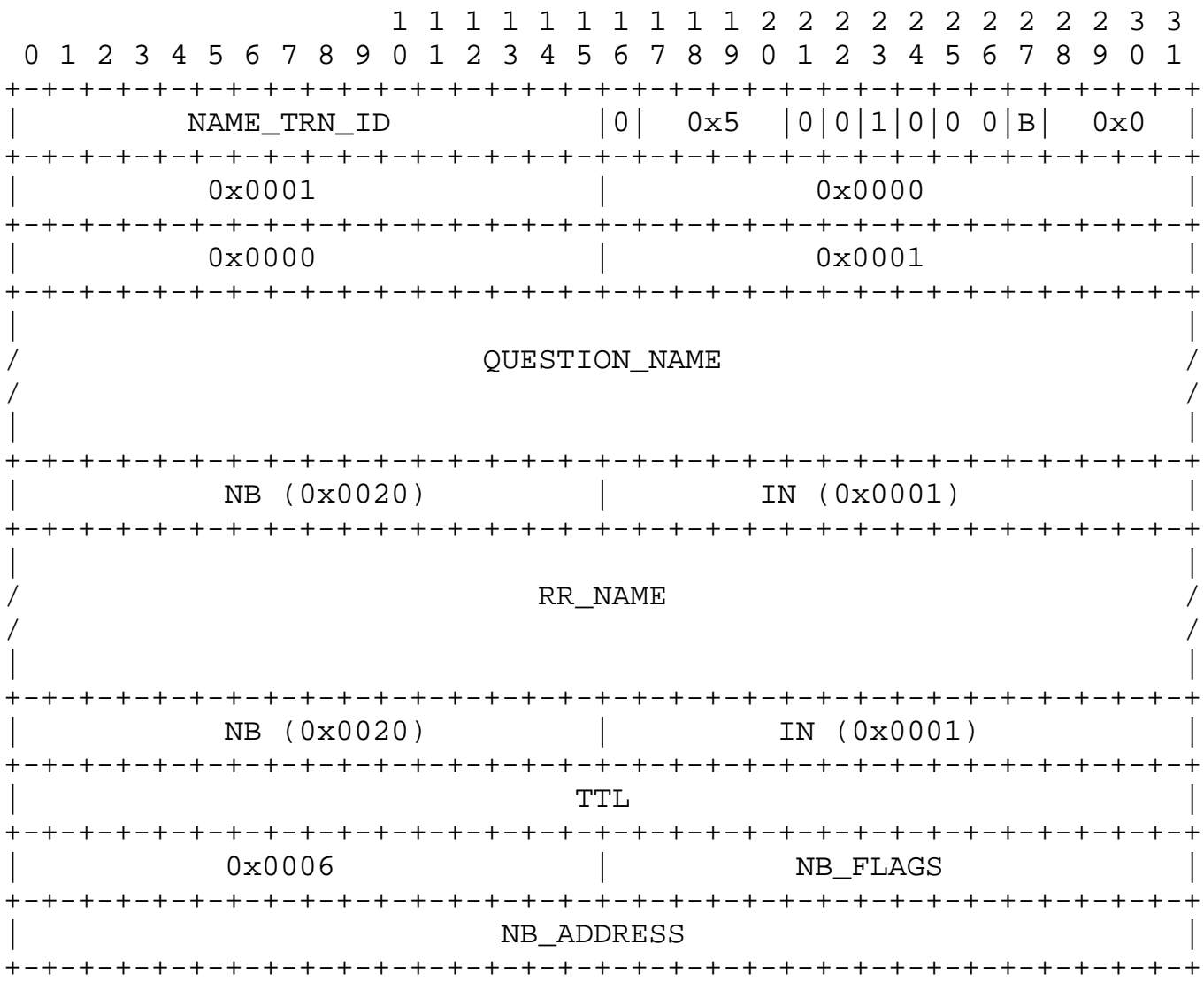

Since the RR_NAME is the same name as the QUESTION_NAME, the RR_NAME representation must use pointers to the QUESTION_NAME name's labels to guarantee the length of the datagram is less than the maximum 576 bytes. See section above on name formats and also page 31 and 32 of RFC 883, Domain Names - Implementation and Specification, for a complete description of compressed name label pointers. 


\subsubsection{NAME OVERWRITE REQUEST \& DEMAND}

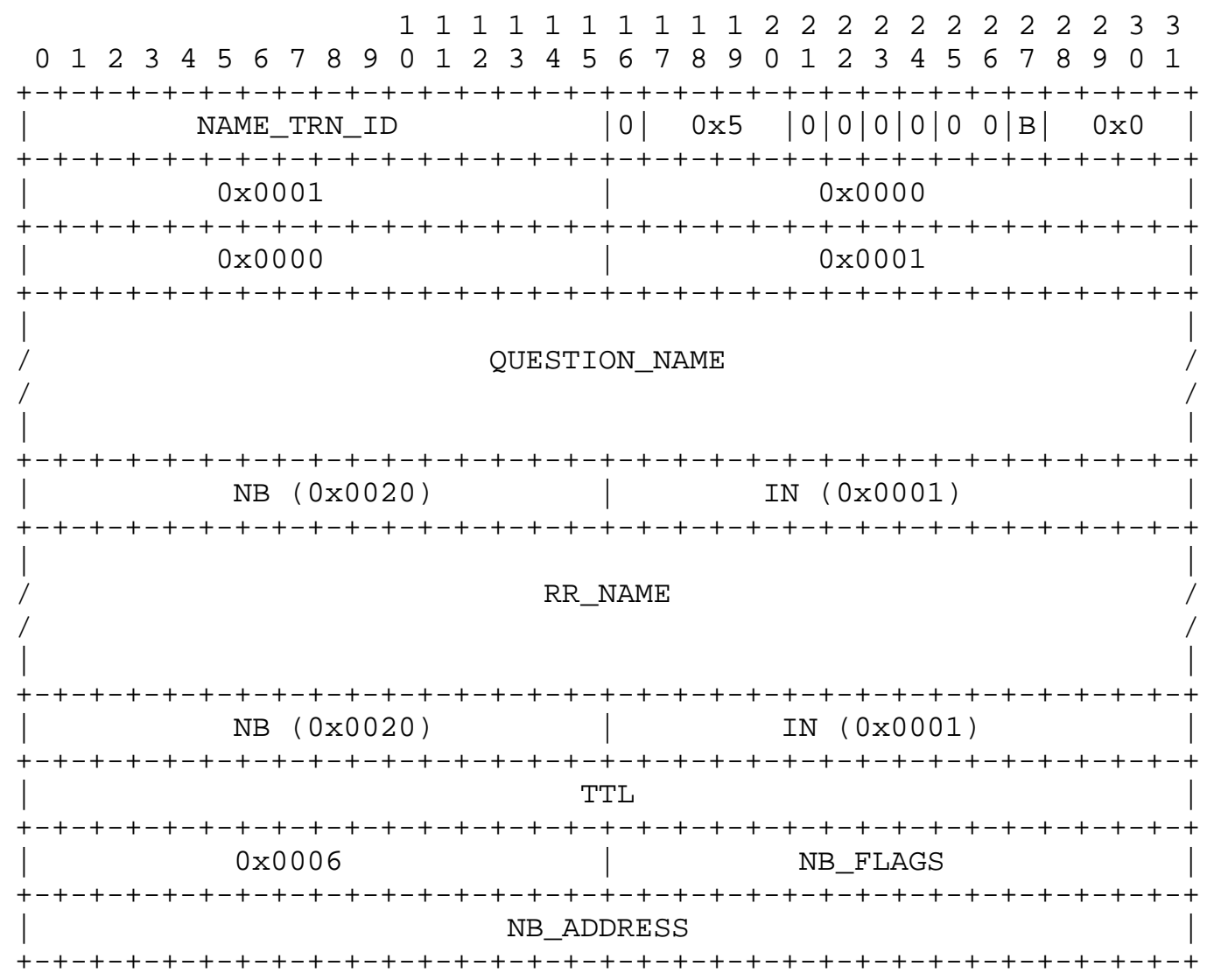




\subsubsection{NAME REFRESH REQUEST}

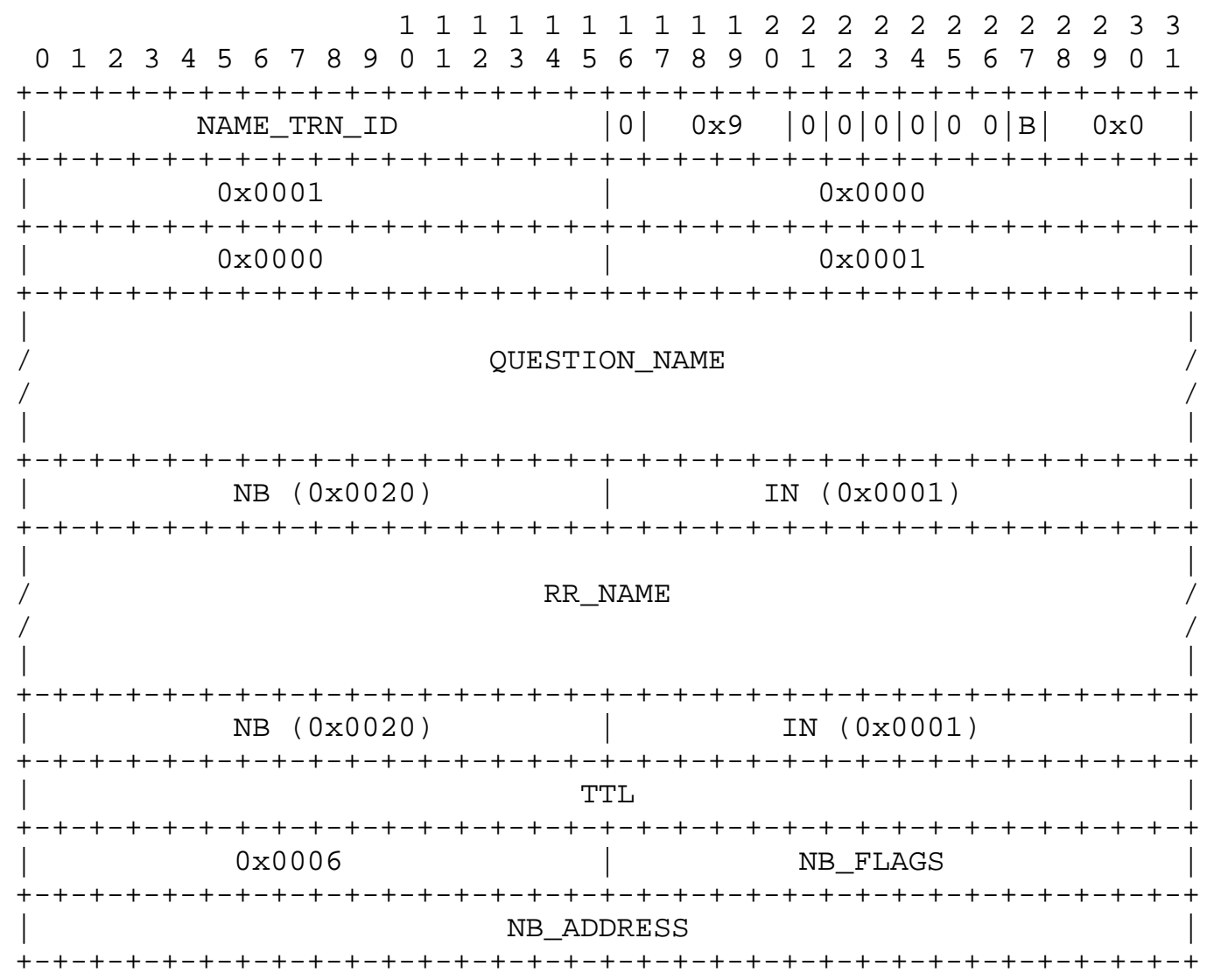




\subsubsection{POSITIVE NAME REGISTRATION RESPONSE}

$\begin{array}{lllllllllllllllllllllll}1 & 1 & 1 & 1 & 1 & 1 & 1 & 1 & 1 & 1 & 1 & 2 & 2 & 2 & 2 & 2 & 2 & 2 & 2 & 2 & 2 & 3 & 3\end{array}$

$\begin{array}{llllllllllllllllllllllllllllllll}0 & 1 & 2 & 3 & 4 & 5 & 6 & 7 & 8 & 9 & 0 & 1 & 2 & 3 & 4 & 5 & 6 & 7 & 8 & 9 & 0 & 1 & 2 & 3 & 4 & 5 & 6 & 7 & 8 & 9 & 0 & 1\end{array}$

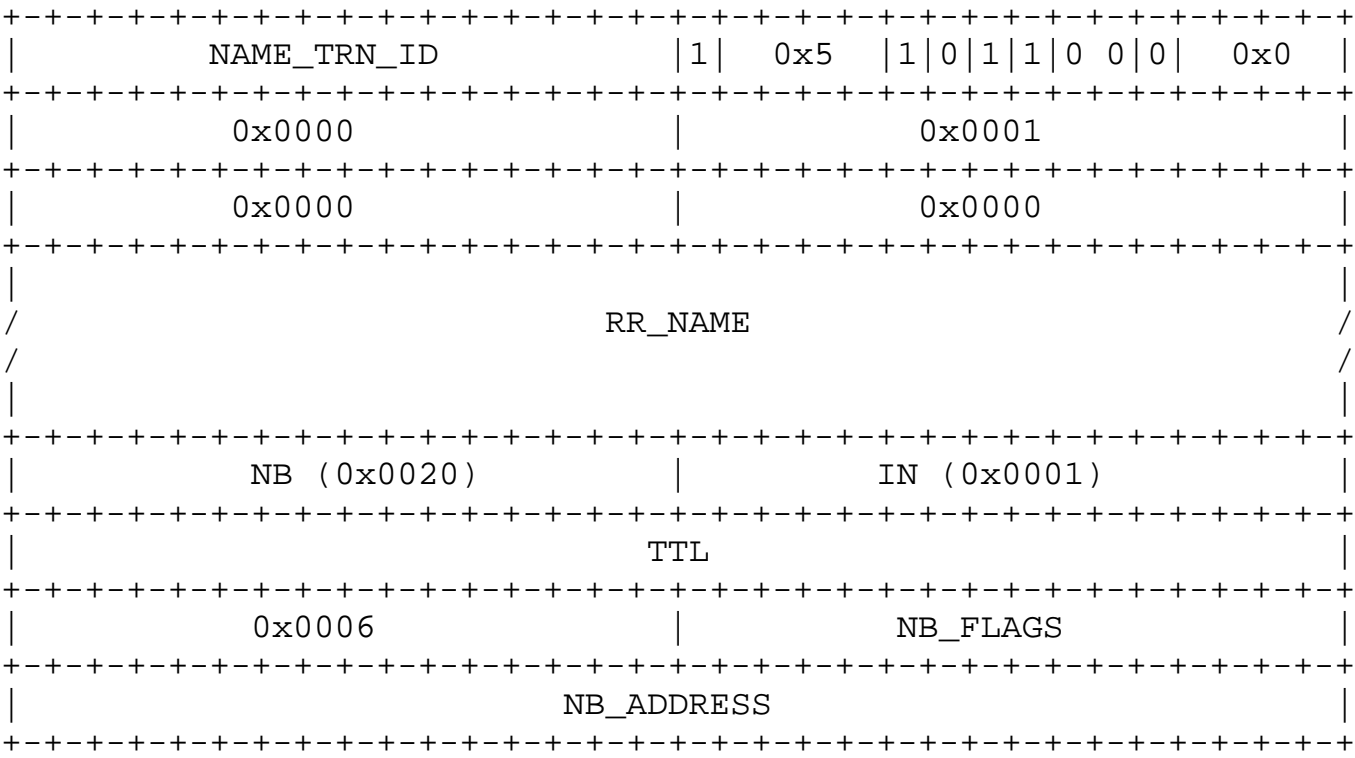

\subsubsection{NEGATIVE NAME REGISTRATION RESPONSE}

$\begin{array}{lllllllllllllllllllllll}1 & 1 & 1 & 1 & 1 & 1 & 1 & 1 & 1 & 1 & 2 & 2 & 2 & 2 & 2 & 2 & 2 & 2 & 2 & 2 & 3 & 3\end{array}$

$\begin{array}{llllllllllllllllllllllllllllllll}0 & 1 & 2 & 3 & 4 & 5 & 6 & 7 & 8 & 9 & 0 & 1 & 2 & 3 & 4 & 5 & 6 & 7 & 8 & 9 & 0 & 1 & 2 & 3 & 4 & 5 & 6 & 7 & 8 & 9 & 0 & 1\end{array}$

$+-+-+-+-+-+-+-+-+-+-+-+-+-+-+-+-+-+-+-+-+-+-+-+-+-+-+-+-+-+-+-+-+$

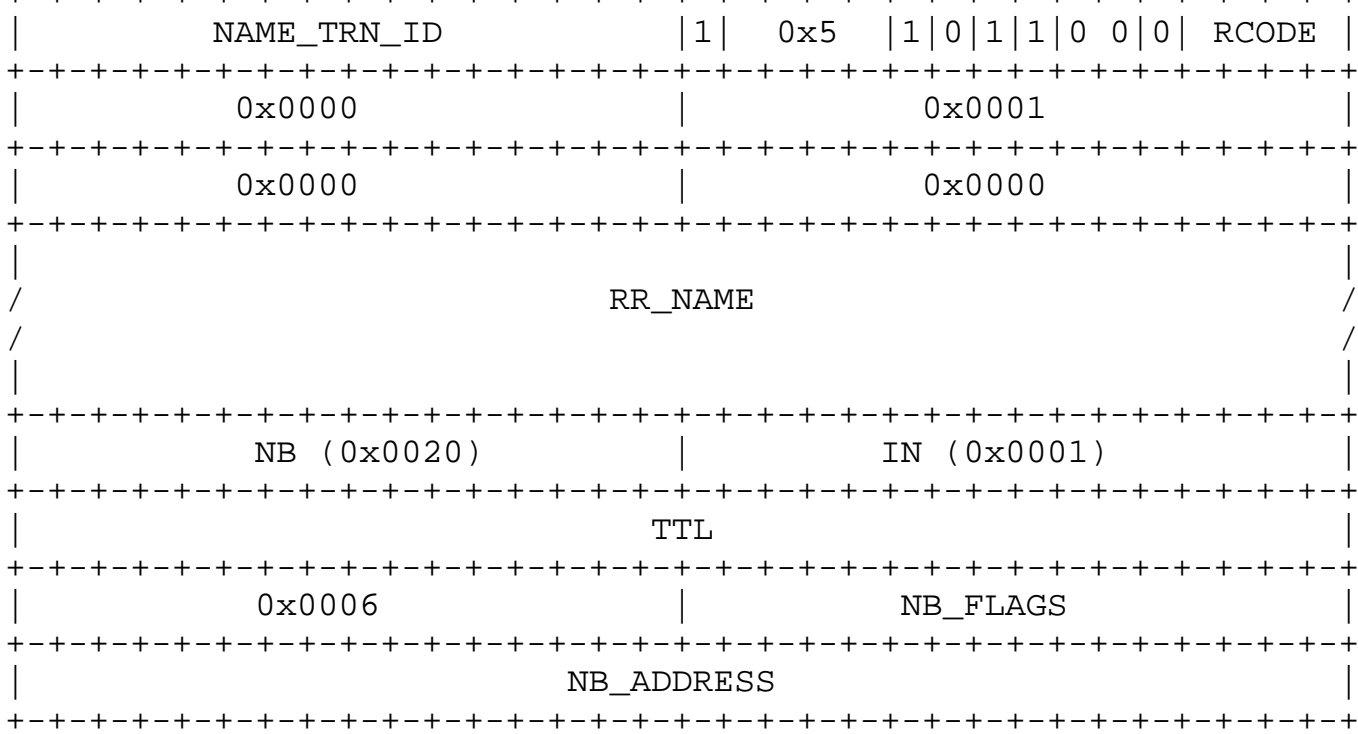


RCODE field values:

\begin{tabular}{|c|c|c|}
\hline Symbol & Value & Description: \\
\hline FMT_ERR & $0 \times 1$ & Request was invalidly \\
\hline SRV_ERR & $0 \times 2$ & $\begin{array}{l}\text { Server failure. Problem with NBNS, cannot } \\
\text { process name. }\end{array}$ \\
\hline IMP_ERR & $0 \times 4$ & $\begin{array}{l}\text { Unsupported request error. Allowable only } \\
\text { for challenging NBNS when gets an Update type } \\
\text { registration request. }\end{array}$ \\
\hline RFS_ERR & $0 \times 5$ & $\begin{array}{l}\text { Refused error. For policy reasons server } \\
\text { will not register this name from this host. }\end{array}$ \\
\hline ACT_ERR & $0 \times 6$ & Active error. Name is owned by another node. \\
\hline CFT_ERR & $0 \times 7$ & $\begin{array}{l}\text { Name in conflict error. A UNIQUE name is } \\
\text { owned by more than one node. }\end{array}$ \\
\hline
\end{tabular}

\subsubsection{END-NODE CHALLENGE REGISTRATION RESPONSE}

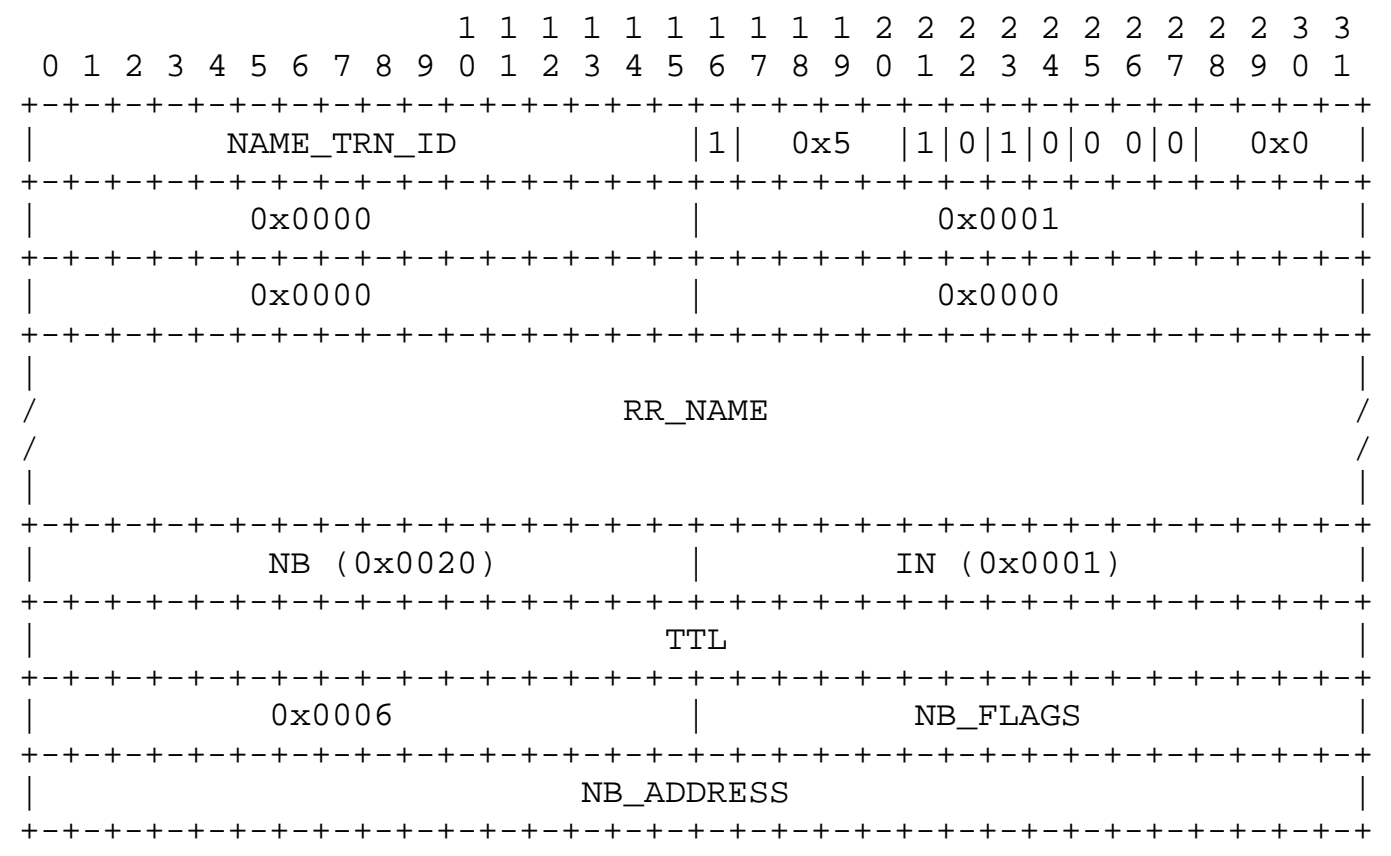




\subsubsection{NAME CONFLICT DEMAND}

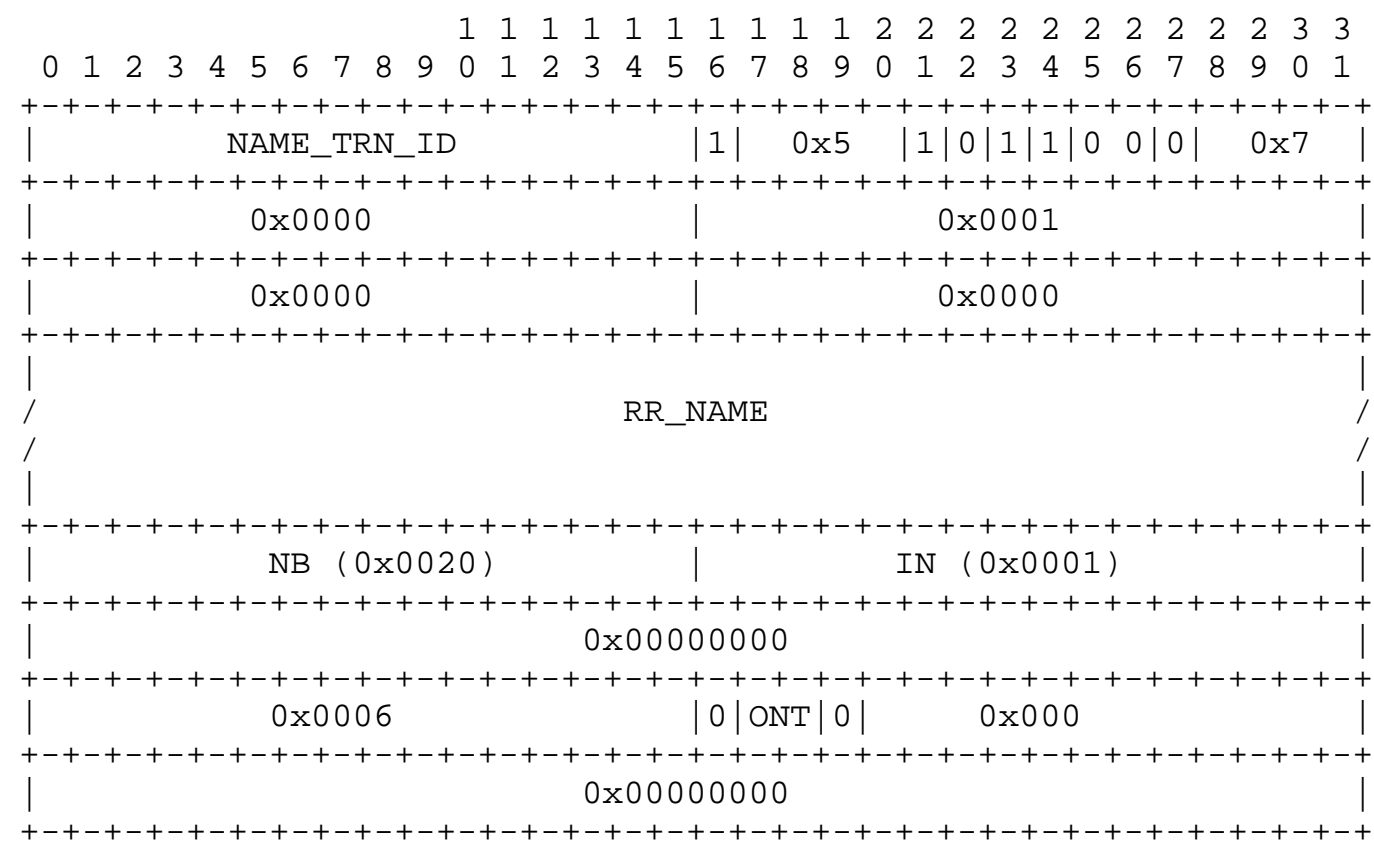

This packet is identical to a NEGATIVE NAME REGISTRATION RESPONSE with RCODE = CFT_ERR. 


\subsubsection{NAME RELEASE REQUEST \& DEMAND}

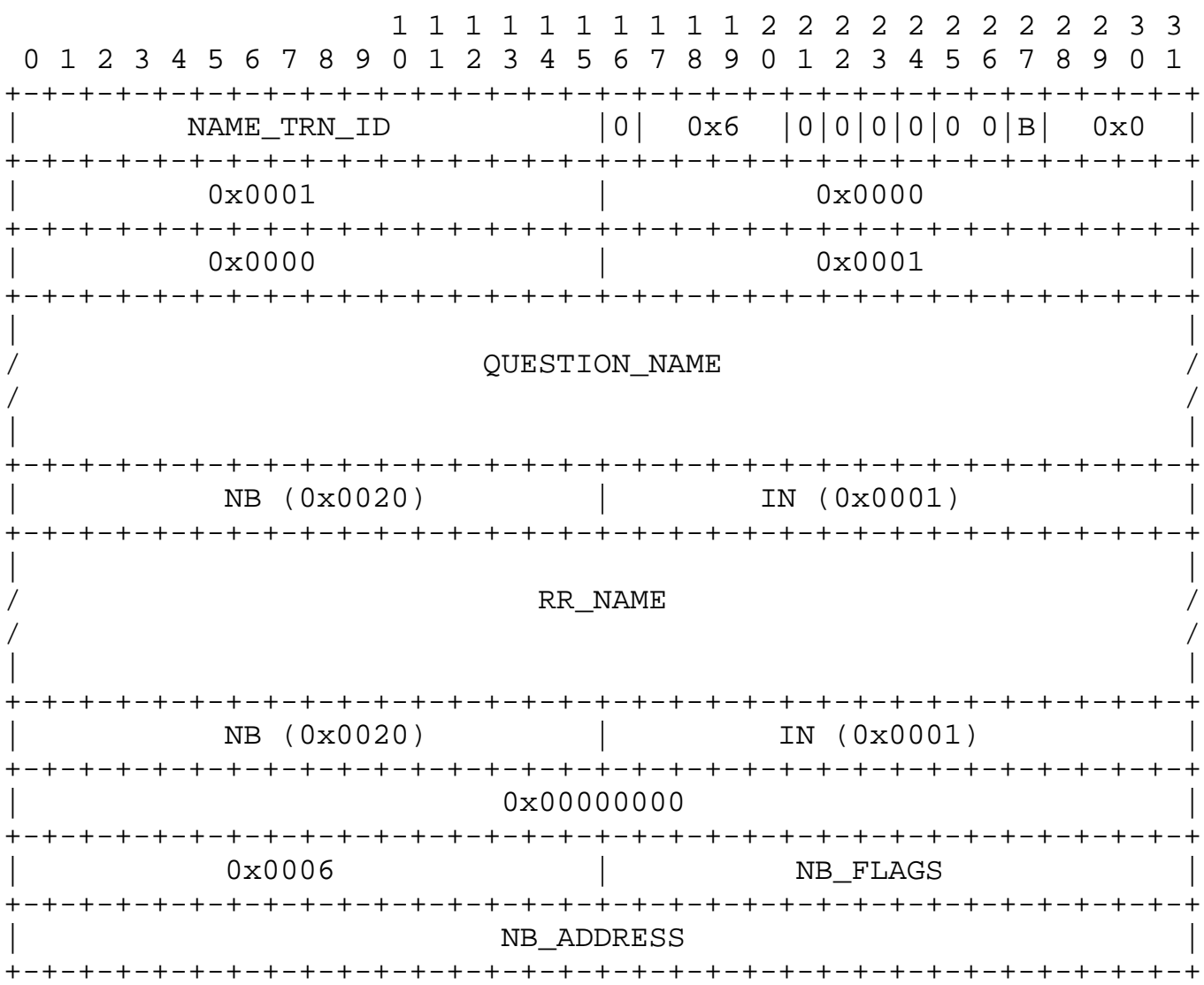

Since the RR_NAME is the same name as the QUESTION_NAME, the RR_NAME representation must use label string pointers to the QUESTION_NAME labels to guarantee the length of the datagram is less than the maximum 576 bytes. This is the same condition as with the NAME REGISTRATION REQUEST. 


\subsubsection{POSITIVE NAME RELEASE RESPONSE}

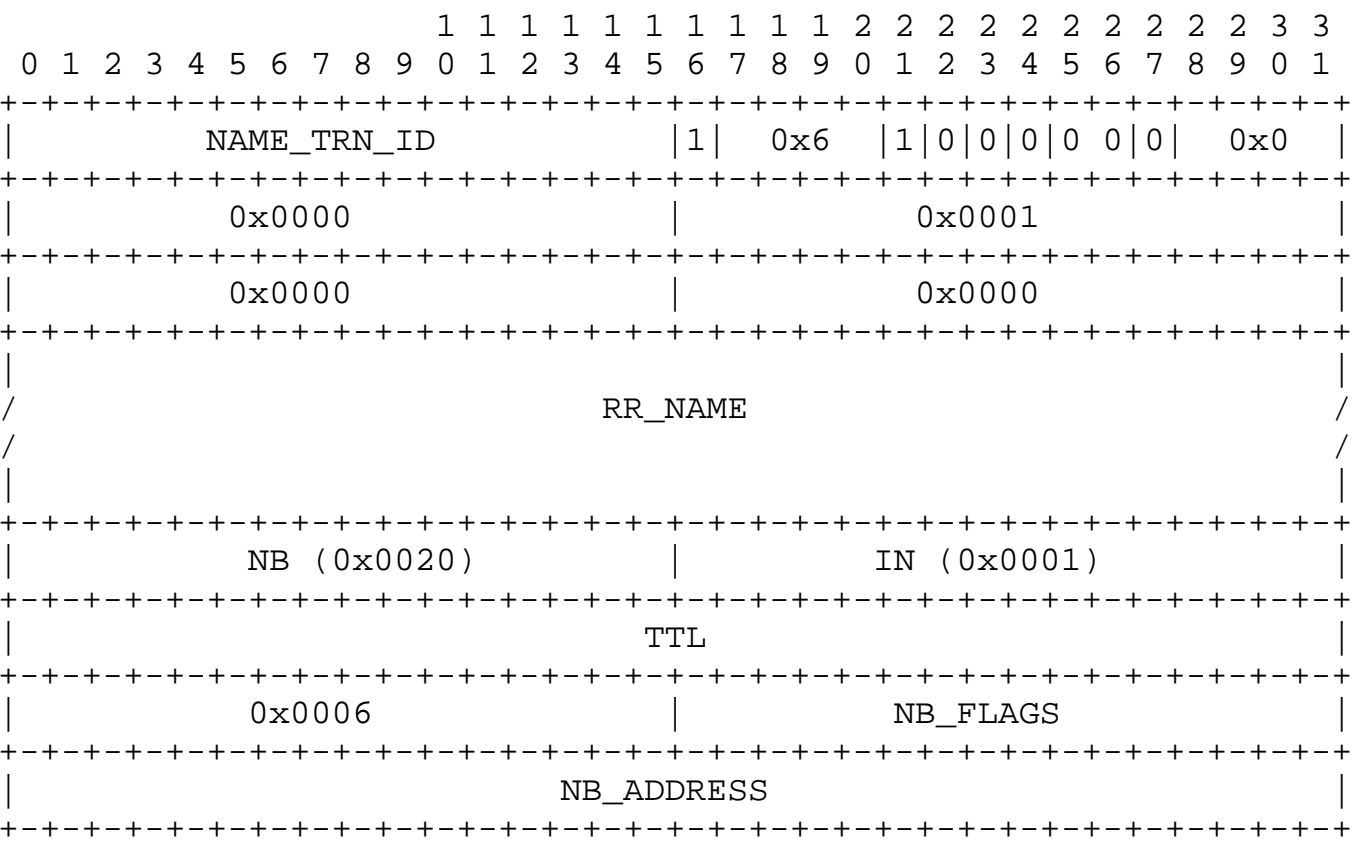

\subsubsection{NEGATIVE NAME RELEASE RESPONSE}

$\begin{array}{llllllllllllllllllllll}1 & 1 & 1 & 1 & 1 & 1 & 1 & 1 & 1 & 1 & 2 & 2 & 2 & 2 & 2 & 2 & 2 & 2 & 2 & 2 & 3 & 3\end{array}$

$\begin{array}{llllllllllllllllllllllllllllllll}0 & 1 & 2 & 3 & 4 & 5 & 6 & 7 & 8 & 9 & 0 & 1 & 2 & 3 & 4 & 5 & 6 & 7 & 8 & 9 & 0 & 1 & 2 & 3 & 4 & 5 & 6 & 7 & 8 & 9 & 0 & 1\end{array}$

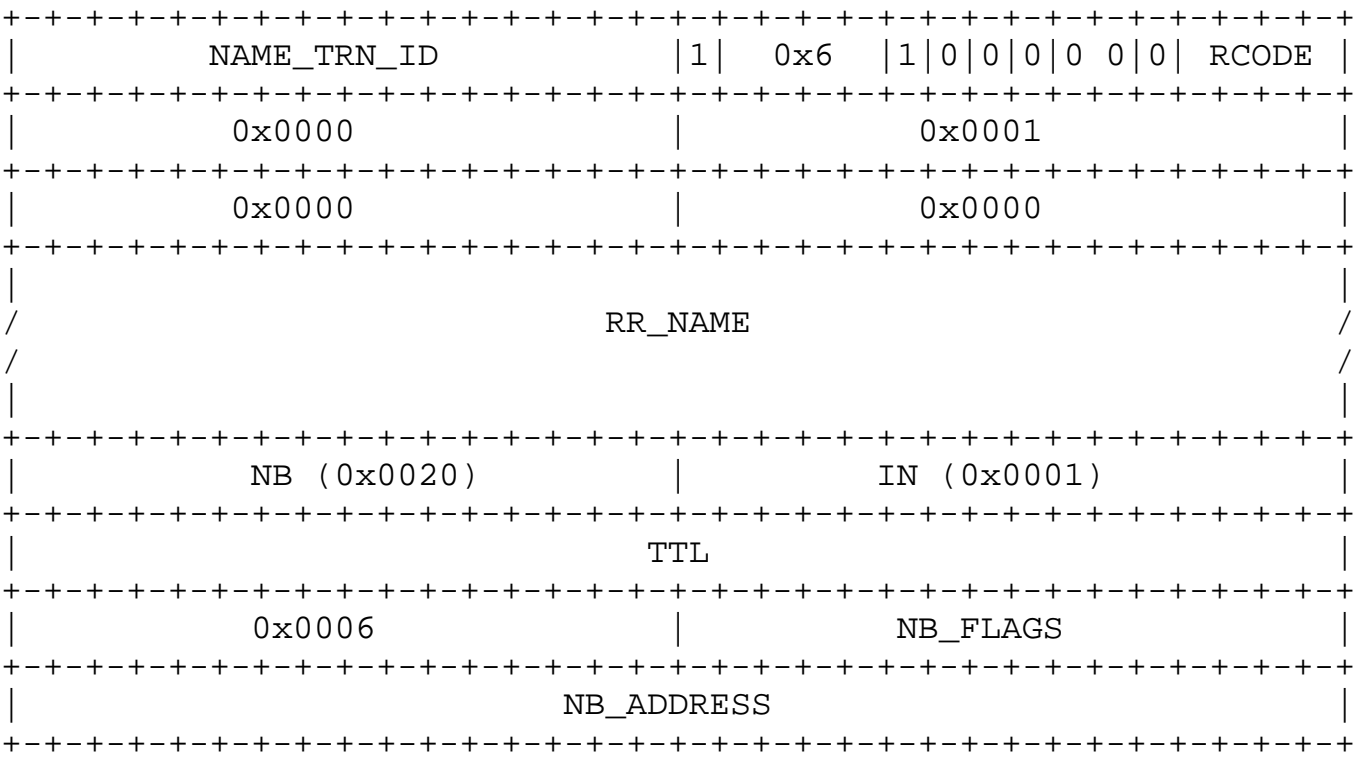


RCODE field values:

\begin{tabular}{|c|c|c|}
\hline Symbol & Value & Description: \\
\hline FMT_ERR & $0 \times 1$ & $\begin{array}{l}\text { Format Error. Request was invalidly } \\
\text { formatted. }\end{array}$ \\
\hline SRV_ERR & $0 \times 2$ & $\begin{array}{l}\text { Server failure. Problem with NBNS, cannot } \\
\text { process name. }\end{array}$ \\
\hline RFS_ERR & $0 \times 5$ & $\begin{array}{l}\text { Refused error. For policy reasons server } \\
\text { will not release this name from this host. }\end{array}$ \\
\hline$A C T \_E R R$ & $0 \times 6$ & $\begin{array}{l}\text { Active error. Name is owned by another node. } \\
\text { Only that node may release it. A NetBIos } \\
\text { Name server can optionally allow a node to } \\
\text { release a name it does not own. This would } \\
\text { facilitate detection of inactive names for } \\
\text { nodes that went down silently. }\end{array}$ \\
\hline
\end{tabular}

\subsubsection{NAME QUERY REQUEST}

$\begin{array}{lllllllllllllllllllllll}1 & 1 & 1 & 1 & 1 & 1 & 1 & 1 & 1 & 1 & 2 & 2 & 2 & 2 & 2 & 2 & 2 & 2 & 2 & 2 & 3 & 3\end{array}$

$\begin{array}{llllllllllllllllllllllllllllllll}0 & 1 & 2 & 3 & 4 & 5 & 6 & 7 & 8 & 9 & 0 & 1 & 2 & 3 & 4 & 5 & 6 & 7 & 8 & 9 & 0 & 1 & 2 & 3 & 4 & 5 & 6 & 7 & 8 & 9 & 0 & 1\end{array}$

$+-+-+-+-+-+-+-+-+-+-+-+-+-+-+-+-+-+-+-+-+-+-+-+-+-+-+-+-+-+-+-+-+$

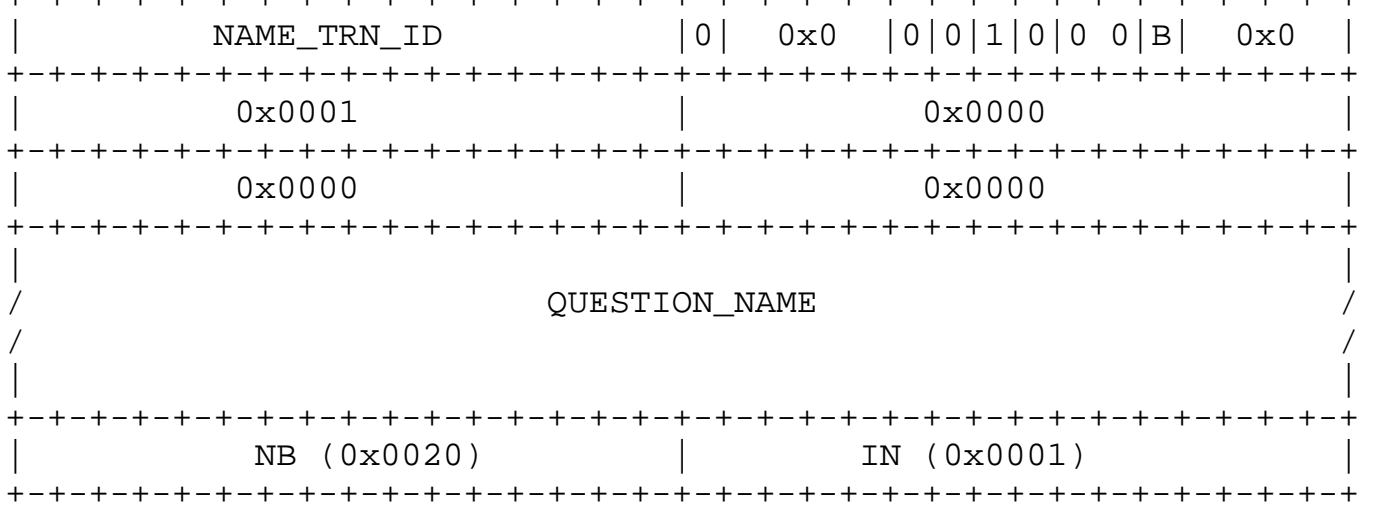




\subsubsection{POSITIVE NAME QUERY RESPONSE}

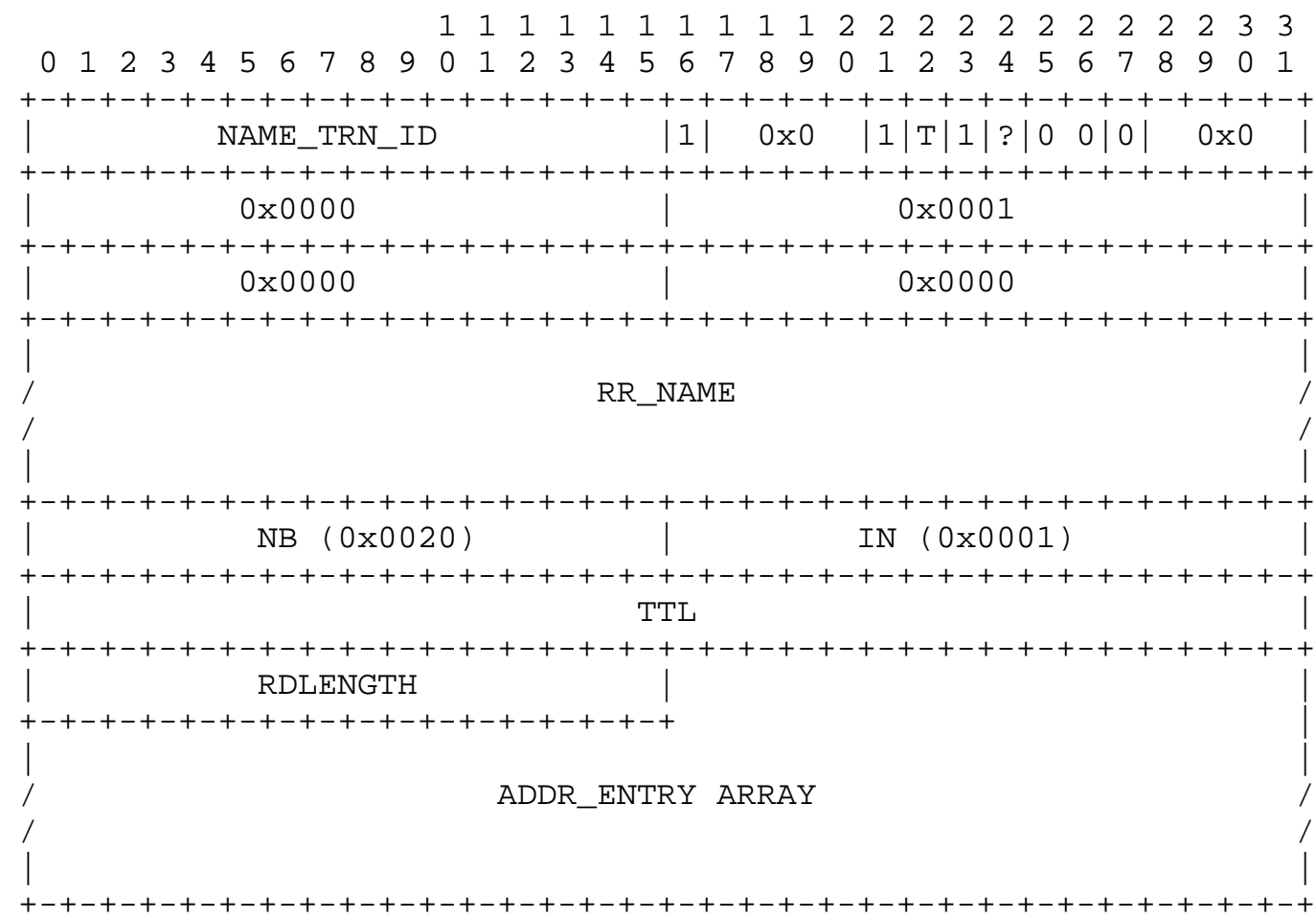

The ADDR_ENTRY ARRAY a sequence of zero or more ADDR_ENTRY records. Each ADDR_ENTRY record represents an owner of a name. For group names there may be multiple entries. However, the list may be incomplete due to packet size limitations. Bit 22, "T", will be set to indicate truncated data.

Each ADDR_ENTRY has the following format:

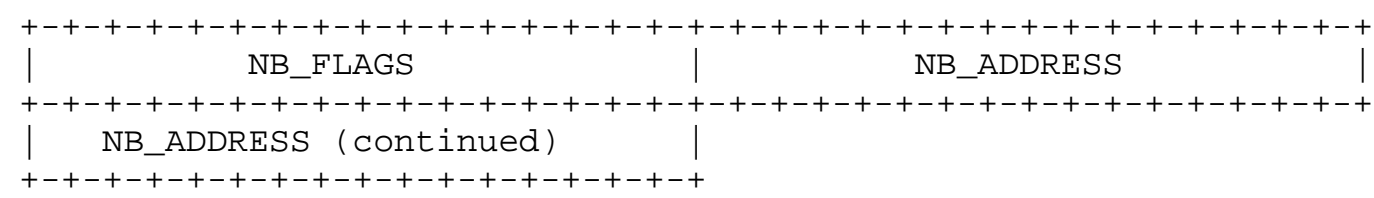




\subsubsection{NEGATIVE NAME QUERY RESPONSE}

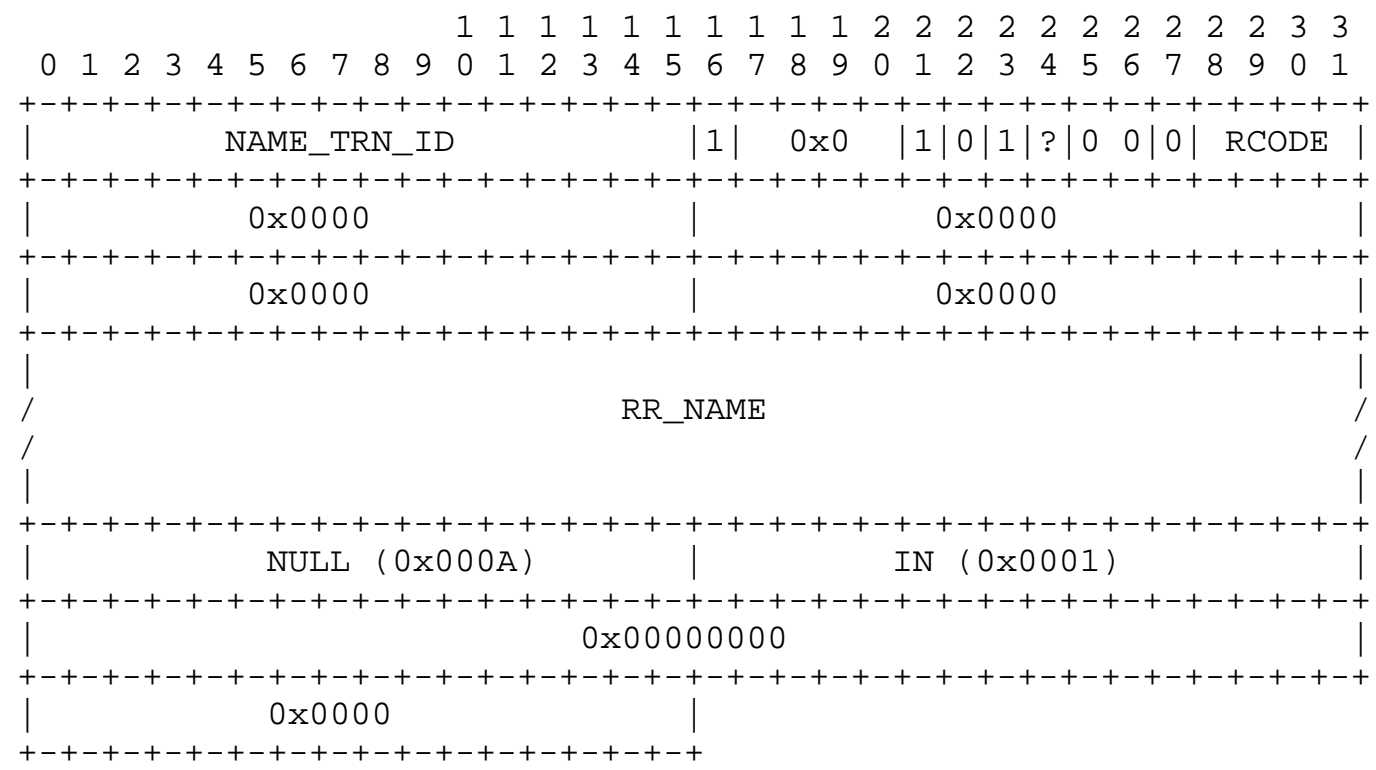

RCODE field values:

Symbol Value Description

FMT_ERR 0x1 Format Error. Request was invalidly

SRV_ERR 0x2 Server failure. Problem with NBNS, cannot process name.

NAM_ERR $0 \times 3$ Name Error. The name requested does not

IMP_ERR 0x4 Unsupported request error. Allowable only for challenging NBNS when gets an Update type registration request.

RFS_ERR

$0 \times 5$

Refused error. For policy reasons server will not register this name from this host. 


\subsubsection{REDIRECT NAME QUERY RESPONSE}

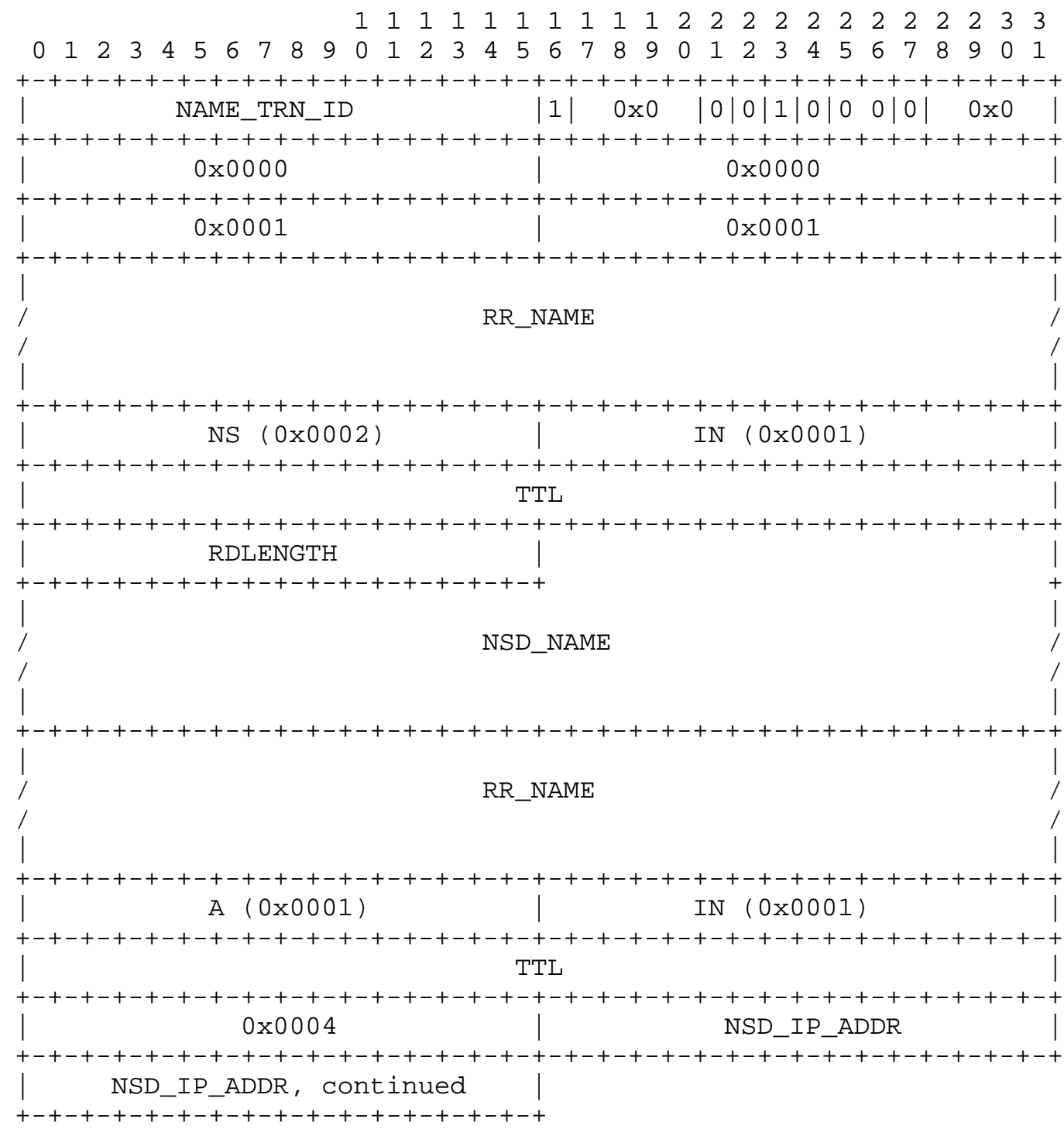

An end node responding to a NAME QUERY REQUEST always responds with the AA and RA bits set for both the NEGATIVE and POSITIVE NAME QUERY RESPONSE packets. An end node never sends a REDIRECT NAME QUERY RESPONSE packet. 
When the requestor receives the REDIRECT NAME QUERY RESPONSE it must reiterate the NAME QUERY REQUEST to the NBNS specified by the NSD_IP_ADDR field of the A type RESOURCE RECORD in the ADDITIONAL section of the response packet. This is an optional packet for the NBNS.

The NSD_NAME and the RR_NAME in the ADDITIONAL section of the response packet are the same name. Space can be optimized if label string pointers are used in the RR_NAME which point to the labels in the NSD_NAME.

The RR_NAME in the AUTHORITY section is the name of the domain the NBNS called by NSD_NAME has authority over.

\subsubsection{WAIT FOR ACKNOWLEDGEMENT (WACK) RESPONSE}

$\begin{array}{llllllllllllllllllllll}1 & 1 & 1 & 1 & 1 & 1 & 1 & 1 & 1 & 1 & 2 & 2 & 2 & 2 & 2 & 2 & 2 & 2 & 2 & 2 & 3 & 3\end{array}$

$\begin{array}{llllllllllllllllllllllllllllllll}0 & 1 & 2 & 3 & 4 & 5 & 6 & 7 & 8 & 9 & 0 & 1 & 2 & 3 & 4 & 5 & 6 & 7 & 8 & 9 & 0 & 1 & 2 & 3 & 4 & 5 & 6 & 7 & 8 & 9 & 0 & 1\end{array}$

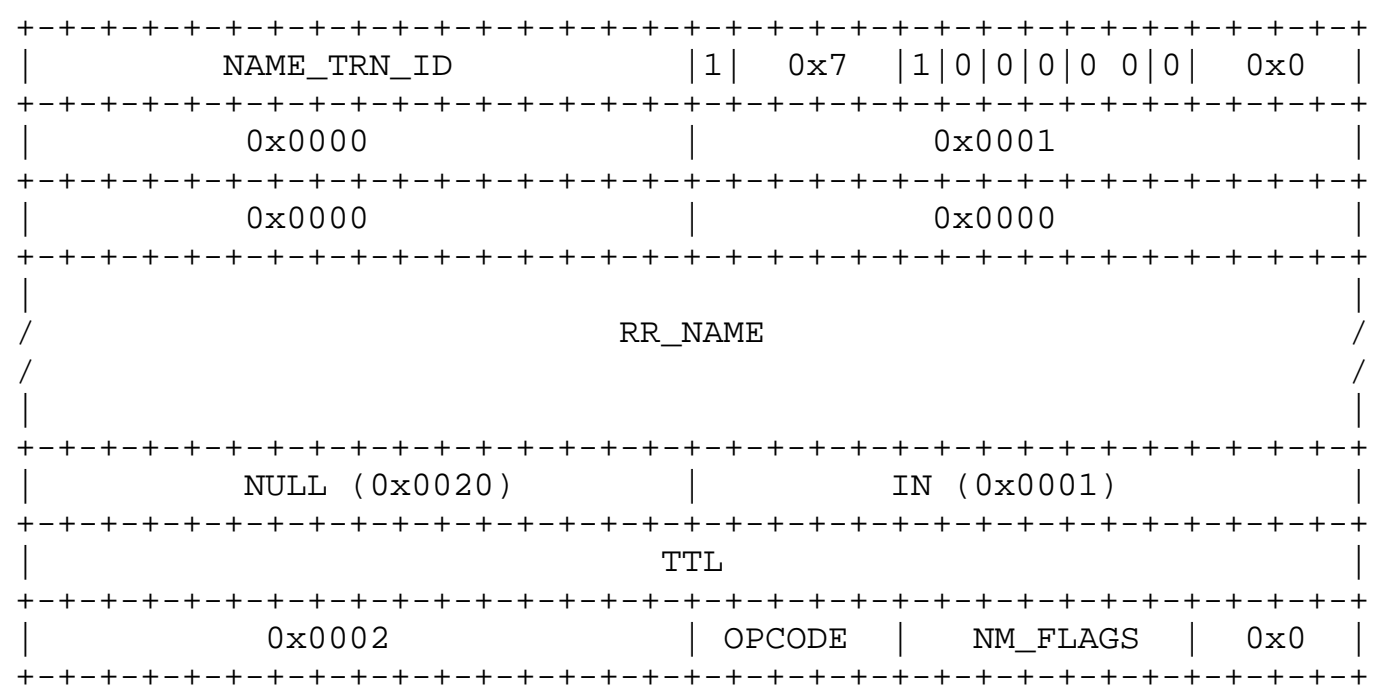


The NAME_TRN_ID of the WACK RESPONSE packet is the same NAME_TRN_ID of the request that the NBNS is telling the requestor to wait longer to complete. The RR_NAME is the name from the request, if any. If no name is available from the request then it is a null name, single byte of zero.

The TTL field of the ResourceRecord is the new time to wait, in seconds, for the request to complete. The RDATA field contains the OPCODE and NM_FLAGS of the request.

A TTL value of 0 means that the NBNS can not estimate the time it may take to complete a response.

\subsubsection{NODE STATUS REQUEST}

$\begin{array}{llllllllllllllllllllll}1 & 1 & 1 & 1 & 1 & 1 & 1 & 1 & 1 & 1 & 2 & 2 & 2 & 2 & 2 & 2 & 2 & 2 & 2 & 2 & 3 & 3\end{array}$

$\begin{array}{llllllllllllllllllllllllllllllll}0 & 1 & 2 & 3 & 4 & 5 & 6 & 7 & 8 & 9 & 0 & 1 & 2 & 3 & 4 & 5 & 6 & 7 & 8 & 9 & 0 & 1 & 2 & 3 & 4 & 5 & 6 & 7 & 8 & 9 & 0 & 1\end{array}$ +-+-+-+-+-+-+-+-+-+-+-+-+-+-+-+-+-+-+-+-+-+-+-+-+-+-+-+-+-+-+-+-+

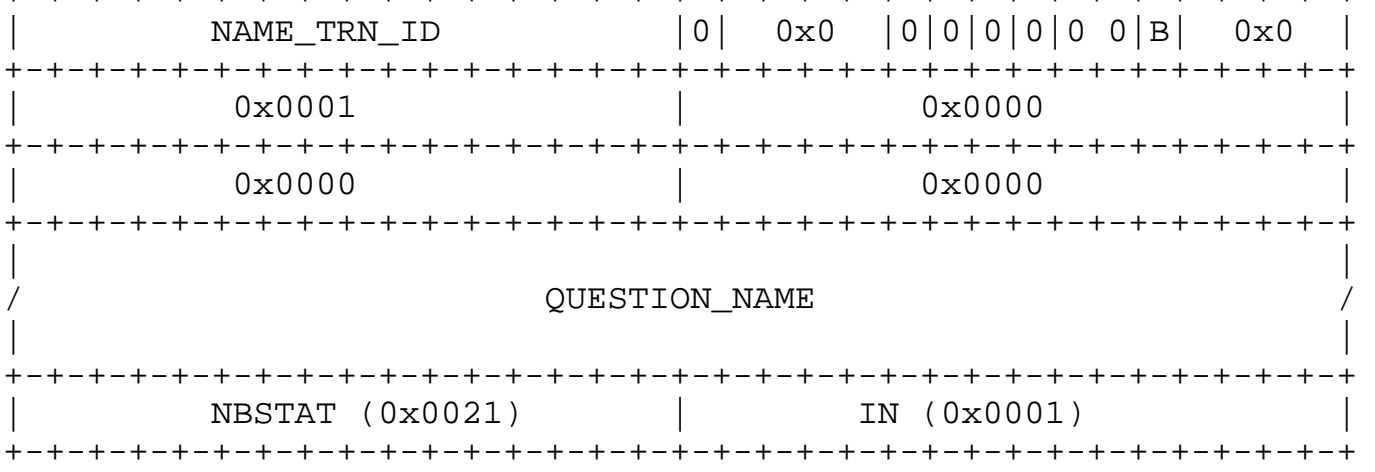




\subsubsection{NODE STATUS RESPONSE}

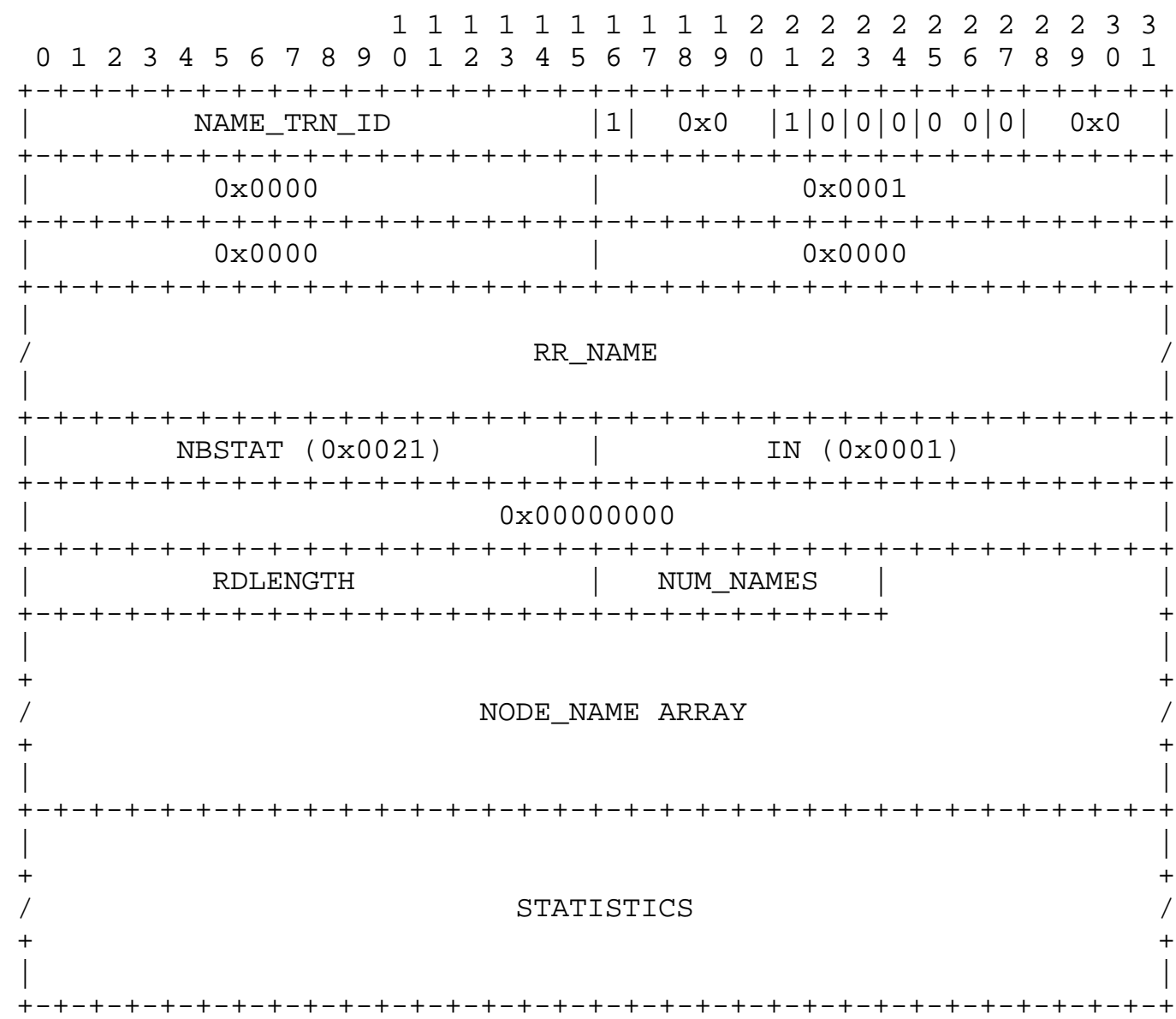

The NODE_NAME ARRAY is an array of zero or more NUM_NAMES entries of NODE_NAME records. Each NODE_NAME entry represents an active name in the same NetBIOS scope as the requesting name in the local name table of the responder. RR_NAME is the requesting name. 
NODE_NAME Entry:

$\begin{array}{llllllllllllllllllllll}1 & 1 & 1 & 1 & 1 & 1 & 1 & 1 & 1 & 1 & 2 & 2 & 2 & 2 & 2 & 2 & 2 & 2 & 2 & 2 & 3 & 3\end{array}$

$\begin{array}{llllllllllllllllllllllllllllllll}0 & 1 & 2 & 3 & 4 & 5 & 6 & 7 & 8 & 9 & 0 & 1 & 2 & 3 & 4 & 5 & 6 & 7 & 8 & 9 & 0 & 1 & 2 & 3 & 4 & 5 & 6 & 7 & 8 & 9 & 0 & 1\end{array}$

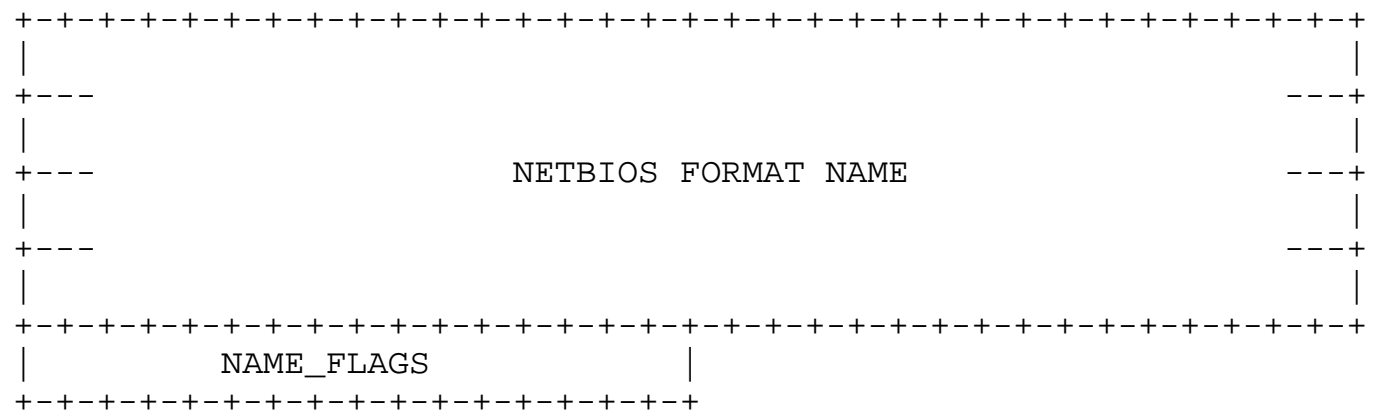

The NAME_FLAGS field:

\begin{tabular}{|c|c|c|c|c|c|c|c|c|c|c|c|c|c|c|c|}
\hline & & & & & & & & & & 1 & 1 & 1 & 1 & 1 & 1 \\
\hline 0 & 1 & 2 & 3 & 4 & 5 & 6 & 7 & 8 & 9 & 0 & 1 & 2 & 3 & 4 & 5 \\
\hline G & & & $\mathrm{DRC}$ & $\mathrm{CNF}$ & $\mathrm{ACT}$ & $|\mathrm{PRM}|$ & & & & $\mathrm{SEF}$ & & & & & \\
\hline
\end{tabular}

The NAME_FLAGS field is defined as:

Symbol Bit(s) Description:

RESERVED 7-15 Reserved for future use. Must be zero (0).

PRM 6 Permanent Name Flag. If one (1) then entry

is for the permanent node name. Flag is zero

(0) for all other names.

ACT 5 Active Name Flag. All entries have this flag set to one (1).

CNF 4 Conflict Flag. If one (1) then name on this node is in conflict.

DRG 3 Deregister Flag. If one (1) then this name

ONT 1,2 Owner Node Type:

$00=\mathrm{B}$ node

$01=\mathrm{P}$ node

$10=\mathrm{M}$ node

11 = Reserved for future use

G

0 Group Name Flag.

If one (1) then the name is a GROUP NetBIOS name.

If zero (0) then it is a UNIQUE NetBIOS name. 
STATISTICS Field of the NODE STATUS RESPONSE:

$\begin{array}{llllllllllllllllllllll}1 & 1 & 1 & 1 & 1 & 1 & 1 & 1 & 1 & 1 & 2 & 2 & 2 & 2 & 2 & 2 & 2 & 2 & 2 & 2 & 3 & 3\end{array}$

$\begin{array}{llllllllllllllllllllllllllllllll}0 & 1 & 2 & 3 & 4 & 5 & 6 & 7 & 8 & 9 & 0 & 1 & 2 & 3 & 4 & 5 & 6 & 7 & 8 & 9 & 0 & 1 & 2 & 3 & 4 & 5 & 6 & 7 & 8 & 9 & 0 & 1\end{array}$ +-+-+-+-+-+-+-+-+-+-+-+-+-+-+-+-+-+-+-+-+-+-+-+-+-+-+-+-+-+-+-+-t

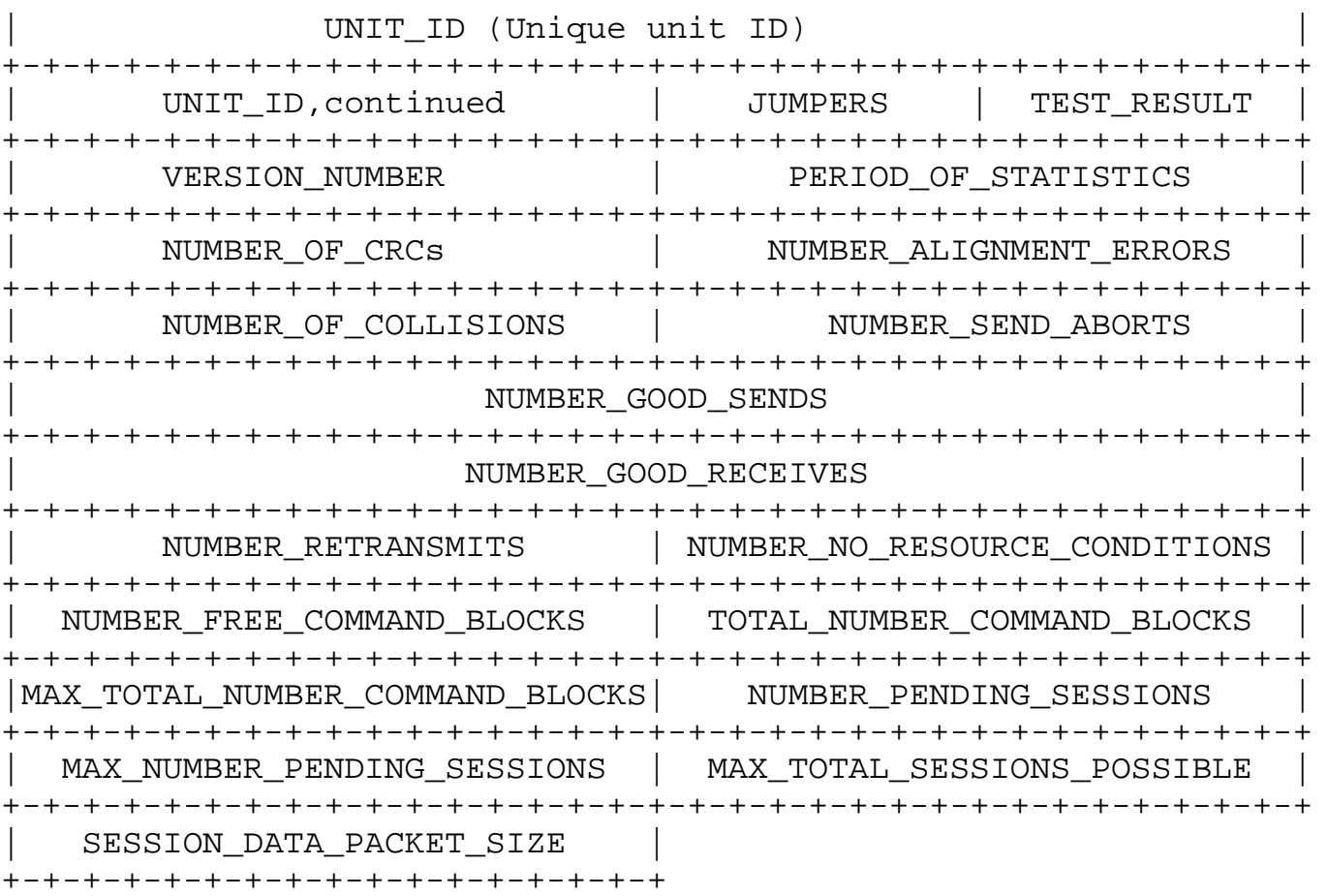

\subsection{SESSION SERVICE PACKETS}

\subsubsection{GENERAL FORMAT OF SESSION PACKETS}

All session service messages are sent over a TCP connection.

All session packets are of the following general structure:

$\begin{array}{llllllllllllllllllllll}1 & 1 & 1 & 1 & 1 & 1 & 1 & 1 & 1 & 1 & 2 & 2 & 2 & 2 & 2 & 2 & 2 & 2 & 2 & 2 & 3 & 3\end{array}$ $\begin{array}{llllllllllllllllllllllllllllllll}0 & 1 & 2 & 3 & 4 & 5 & 6 & 7 & 8 & 9 & 0 & 1 & 2 & 3 & 4 & 5 & 6 & 7 & 8 & 9 & 0 & 1 & 2 & 3 & 4 & 5 & 6 & 7 & 8 & 9 & 0 & 1\end{array}$ +-+-+-+-+-+-+-+-+-+-+-+-+-+-+-+-+-+-+-+-+-+-+-+-+-+-+-+-+-+-+-+-+ \begin{tabular}{c|c|} 
TYPE & FLAGS \\
$\mid$ & TRAILER (Packet Type Dependent)
\end{tabular} \mid

The TYPE, FLAGS, and LENGTH fields are present in every session packet. 
The LENGTH field is the number of bytes following the LENGTH field. In other words, LENGTH is the combined size of the TRAILER field(s). For example, the POSITIVE SESSION RESPONSE packet always has a LENGTH field value of zero (0000) while the RETARGET SESSION RESPONSE always has a LENGTH field value of six $(0006)$.

One of the bits of the FLAGS field acts as an additional, highorder bit for the LENGTH field. Thus the cumulative size of the trailer field(s) may range from 0 to $128 \mathrm{~K}$ bytes.

Session Packet Types (in hexidecimal):

$$
\begin{aligned}
& 00 \text { - SESSION MESSAGE } \\
& 81 \text { - SESSION REQUEST } \\
& 82 \text { - POSITIVE SESSION RESPONSE } \\
& 83 \text { - NEGATIVE SESSION RESPONSE } \\
& 84 \text { - RETARGET SESSION RESPONSE } \\
& 85 \text { - SESSION KEEP ALIVE }
\end{aligned}
$$

Bit definitions of the FLAGS field:

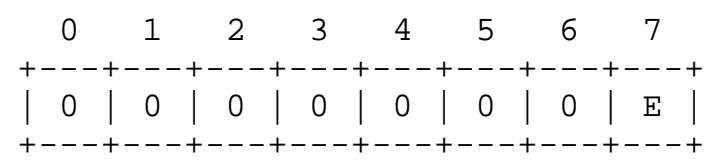

Symbol Bit (s) Description

E 7 Length extension, used as an additional, high-order bit on the LENGTH field.

RESERVED $0-6$ Reserved, must be zero (0)

\subsubsection{SESSION REQUEST PACKET}

$\begin{array}{llllllllllllllllllllll}1 & 1 & 1 & 1 & 1 & 1 & 1 & 1 & 1 & 1 & 2 & 2 & 2 & 2 & 2 & 2 & 2 & 2 & 2 & 2 & 3 & 3\end{array}$

$\begin{array}{llllllllllllllllllllllllllllllll}0 & 1 & 2 & 3 & 4 & 5 & 6 & 7 & 8 & 9 & 0 & 1 & 2 & 3 & 4 & 5 & 6 & 7 & 8 & 9 & 0 & 1 & 2 & 3 & 4 & 5 & 6 & 7 & 8 & 9 & 0 & 1\end{array}$

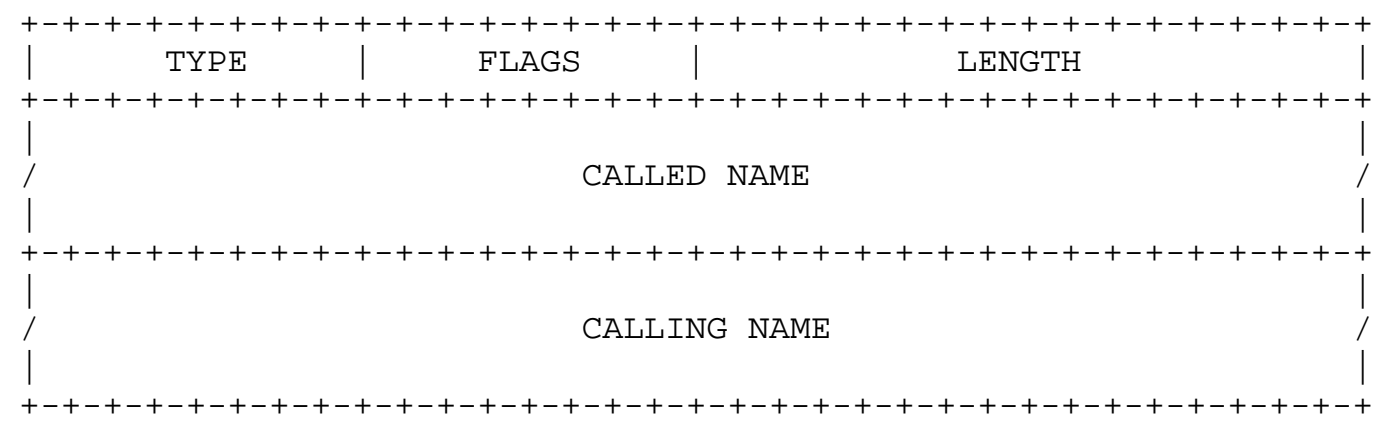




\subsubsection{POSITIVE SESSION RESPONSE PACKET}

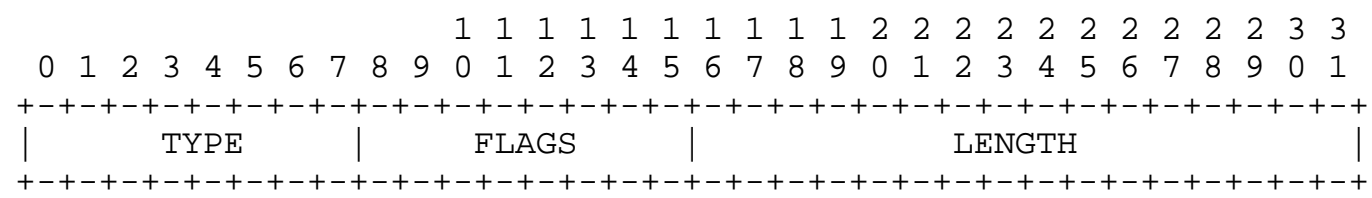

\subsubsection{NEGATIVE SESSION RESPONSE PACKET}

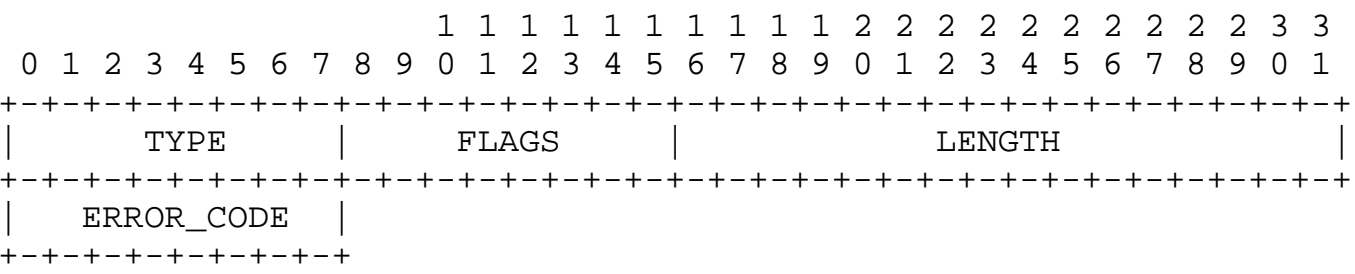

NEGATIVE SESSION RESPONSE packet error code values (in hexidecimal) :

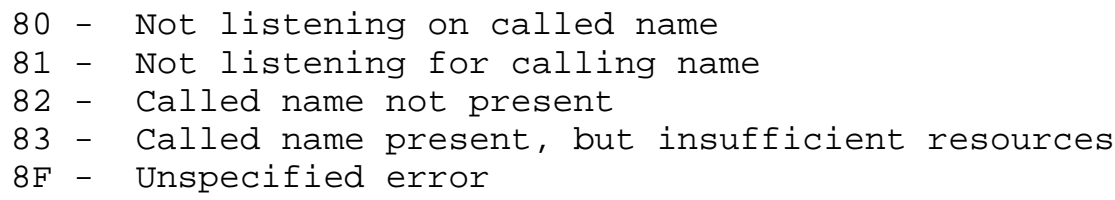

\subsubsection{SESSION RETARGET RESPONSE PACKET}

$\begin{array}{llllllllllllllllllllll}1 & 1 & 1 & 1 & 1 & 1 & 1 & 1 & 1 & 1 & 2 & 2 & 2 & 2 & 2 & 2 & 2 & 2 & 2 & 2 & 3 & 3\end{array}$ $\begin{array}{llllllllllllllllllllllllllllllll}0 & 1 & 2 & 3 & 4 & 5 & 6 & 7 & 8 & 9 & 0 & 1 & 2 & 3 & 4 & 5 & 6 & 7 & 8 & 9 & 0 & 1 & 2 & 3 & 4 & 5 & 6 & 7 & 8 & 9 & 0 & 1\end{array}$

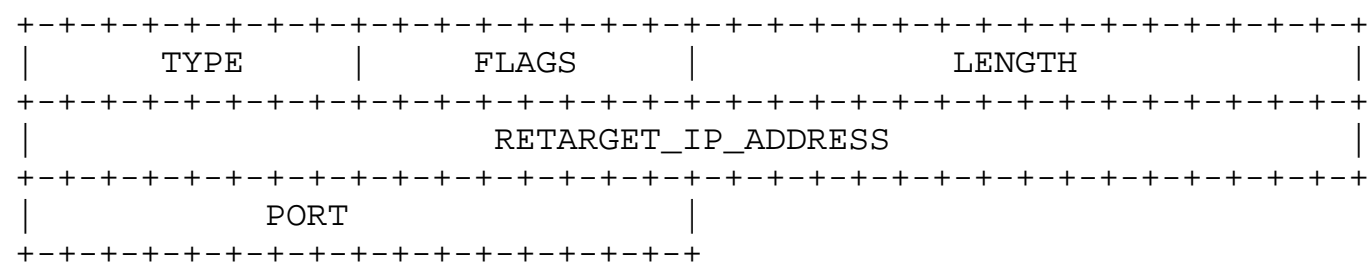




\subsubsection{SESSION MESSAGE PACKET}

$\begin{array}{llllllllllllllllllllll}1 & 1 & 1 & 1 & 1 & 1 & 1 & 1 & 1 & 1 & 2 & 2 & 2 & 2 & 2 & 2 & 2 & 2 & 2 & 2 & 3 & 3\end{array}$

$\begin{array}{llllllllllllllllllllllllllllllll}0 & 1 & 2 & 3 & 4 & 5 & 6 & 7 & 8 & 9 & 0 & 1 & 2 & 3 & 4 & 5 & 6 & 7 & 8 & 9 & 0 & 1 & 2 & 3 & 4 & 5 & 6 & 7 & 8 & 9 & 0 & 1\end{array}$

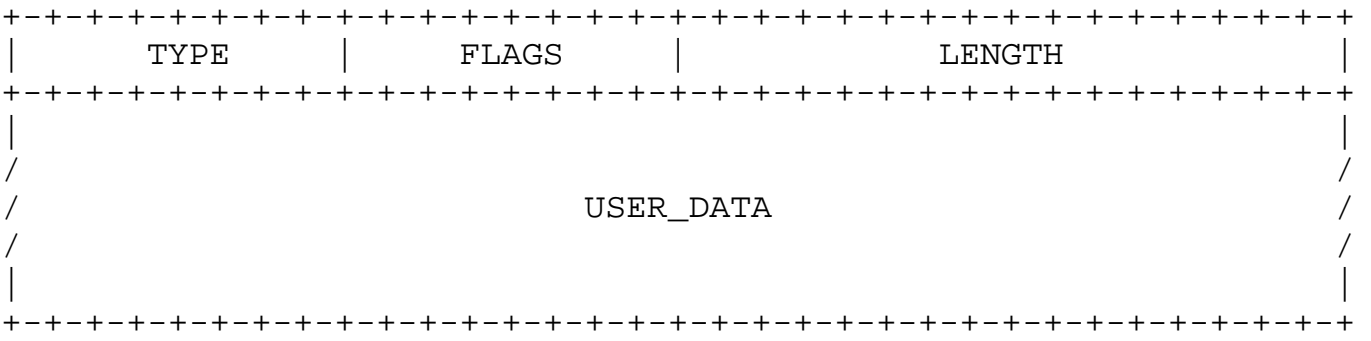

4.3.7. SESSION KEEP ALIVE PACKET

$\begin{array}{llllllllllllllllllllll}1 & 1 & 1 & 1 & 1 & 1 & 1 & 1 & 1 & 1 & 2 & 2 & 2 & 2 & 2 & 2 & 2 & 2 & 2 & 2 & 3 & 3\end{array}$

$\begin{array}{llllllllllllllllllllllllllllllll}0 & 1 & 2 & 3 & 4 & 5 & 6 & 7 & 8 & 9 & 0 & 1 & 2 & 3 & 4 & 5 & 6 & 7 & 8 & 9 & 0 & 1 & 2 & 3 & 4 & 5 & 6 & 7 & 8 & 9 & 0 & 1\end{array}$

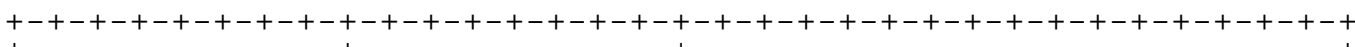

$$
\mid
$$
\begin{tabular}{l|l} 
TYPE & FLAGS
\end{tabular} LENGTH

\subsection{DATAGRAM SERVICE PACKETS}

\subsubsection{NetBIOS DATAGRAM HEADER}

$\begin{array}{llllllllllllllllllllll}1 & 1 & 1 & 1 & 1 & 1 & 1 & 1 & 1 & 1 & 2 & 2 & 2 & 2 & 2 & 2 & 2 & 2 & 2 & 2 & 3 & 3\end{array}$ $\begin{array}{lllllllllllllllllllllllllllllllll}0 & 1 & 2 & 3 & 4 & 5 & 6 & 7 & 8 & 9 & 0 & 1 & 2 & 3 & 4 & 5 & 6 & 7 & 8 & 9 & 0 & 1 & 2 & 3 & 4 & 5 & 6 & 7 & 8 & 9 & 0 & 1\end{array}$

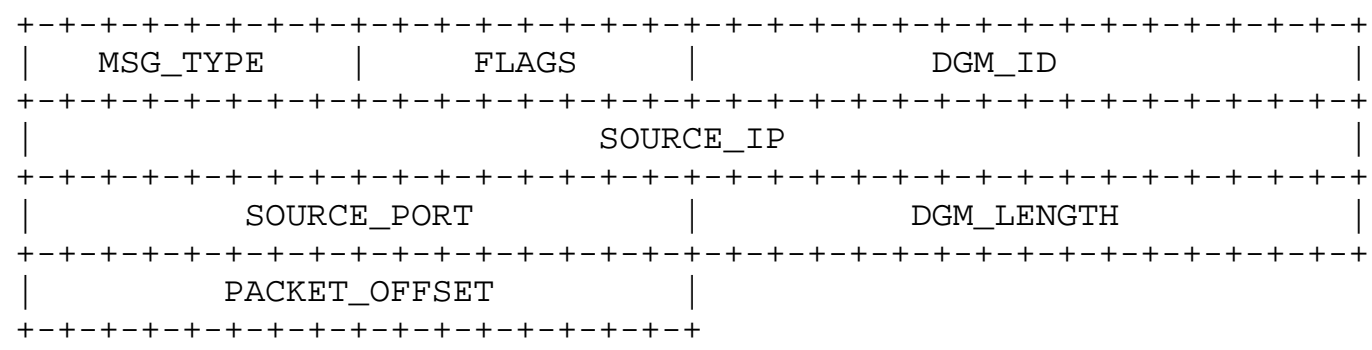

MSG_TYPE values (in hexidecimal):

$$
\begin{aligned}
& 10 \text { - DIRECT_UNIQUE DATAGRAM } \\
& 11 \text { - DIRECT_GROUP DATAGRAM } \\
& 12 \text { - BROADCAST DATAGRAM } \\
& 13 \text { - DATAGRAM ERROR } \\
& 14 \text { - DATAGRAM QUERY REQUEST } \\
& 15 \text { - DATAGRAM POSITIVE QUERY RESPONSE } \\
& 16 \text { - DATAGRAM NEGATIVE QUERY RESPONSE }
\end{aligned}
$$


Bit definitions of the FLAGS field:

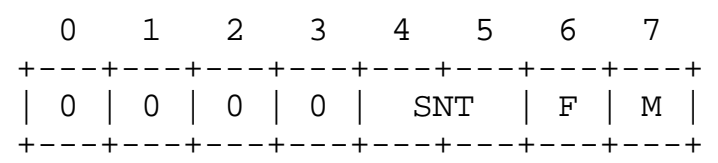

Symbol Bit (s) Description

M 7 MORE flag, If set then more NetBIOS datagram fragments follow.

F

6 FIRST packet flag, If set then this is first (and possibly only) fragment of NetBIos datagram

SNT 4,5 Source End-Node type:

$$
\begin{aligned}
& 00=\mathrm{B} \text { node } \\
& 01=\mathrm{P} \text { node } \\
& 10=\mathrm{M} \text { node } \\
& 11=\mathrm{NBDD}
\end{aligned}
$$

RESERVED $0-3$ Reserved, must be zero (0)

4.4.2. DIRECT_UNIQUE, DIRECT_GROUP, \& BROADCAST DATAGRAM

$\begin{array}{llllllllllllllllllllll}1 & 1 & 1 & 1 & 1 & 1 & 1 & 1 & 1 & 1 & 2 & 2 & 2 & 2 & 2 & 2 & 2 & 2 & 2 & 2 & 3 & 3\end{array}$ $\begin{array}{llllllllllllllllllllllllllllllll}0 & 1 & 2 & 3 & 4 & 5 & 6 & 7 & 8 & 9 & 0 & 1 & 2 & 3 & 4 & 5 & 6 & 7 & 8 & 9 & 0 & 1 & 2 & 3 & 4 & 5 & 6 & 7 & 8 & 9 & 0 & 1\end{array}$

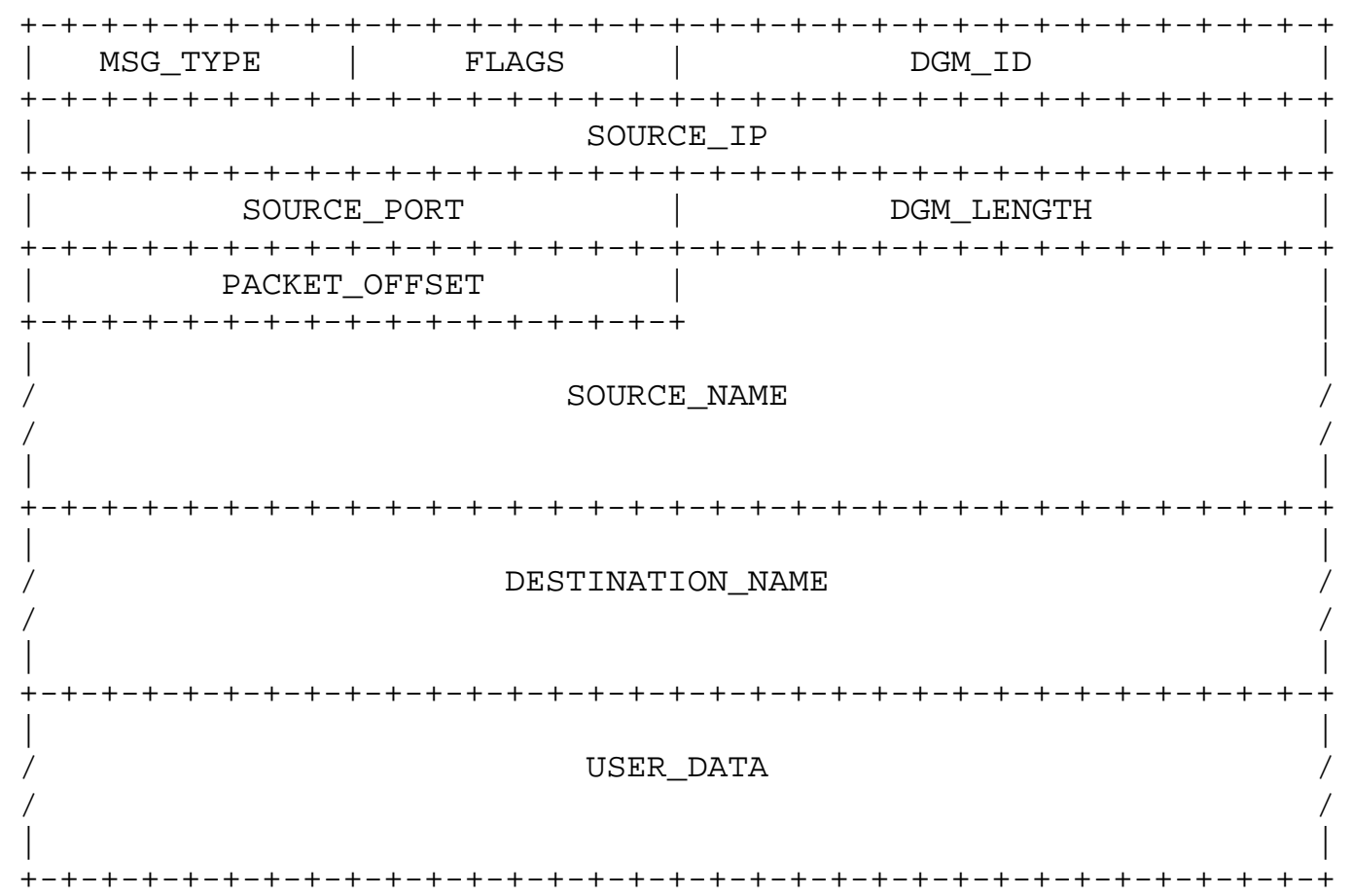




\subsubsection{DATAGRAM ERROR PACKET}

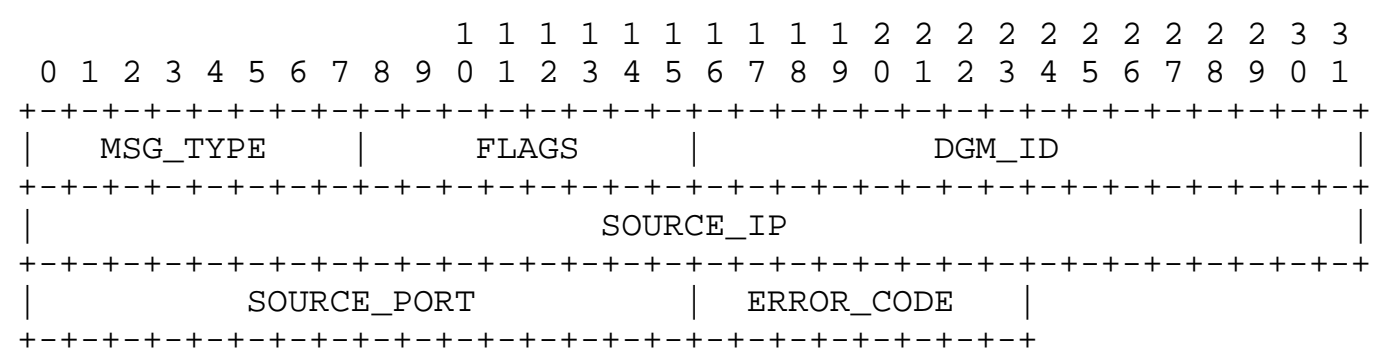

ERROR_CODE values (in hexidecimal):

82 - DESTINATION NAME NOT PRESENT

83 - INVALID SOURCE NAME FORMAT

84 - INVALID DESTINATION NAME FORMAT

4.4.4. DATAGRAM QUERY REQUEST

$\begin{array}{llllllllllllllllllllll}1 & 1 & 1 & 1 & 1 & 1 & 1 & 1 & 1 & 1 & 2 & 2 & 2 & 2 & 2 & 2 & 2 & 2 & 2 & 2 & 3 & 3\end{array}$

$\begin{array}{llllllllllllllllllllllllllllllll}0 & 1 & 2 & 3 & 4 & 5 & 6 & 7 & 8 & 9 & 0 & 1 & 2 & 3 & 4 & 5 & 6 & 7 & 8 & 9 & 0 & 1 & 2 & 3 & 4 & 5 & 6 & 7 & 8 & 9 & 0 & 1\end{array}$

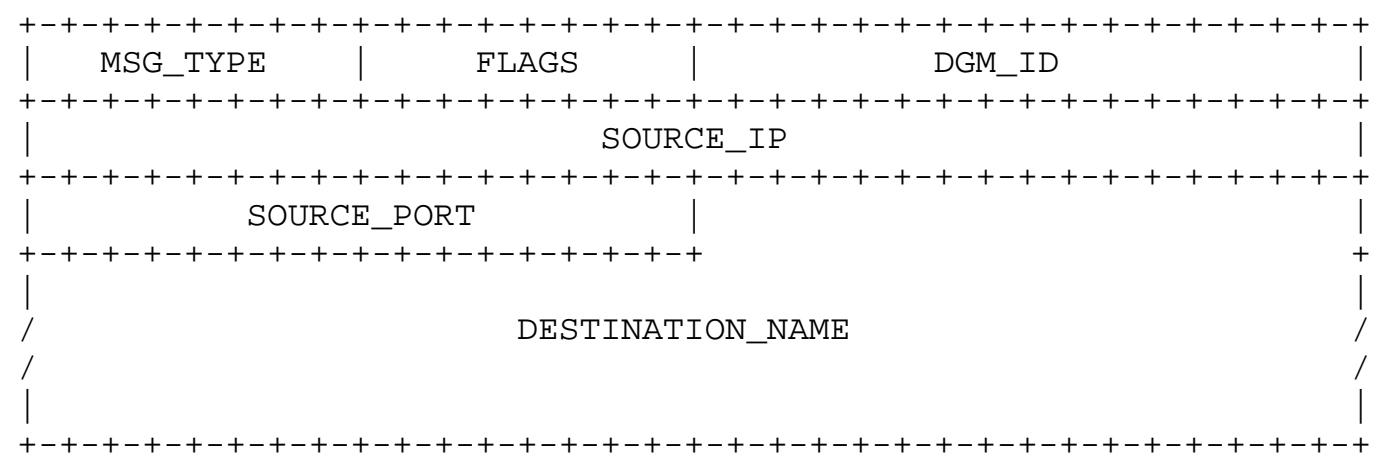

4.4.5. DATAGRAM POSITIVE AND NEGATIVE QUERY RESPONSE

$\begin{array}{llllllllllllllllllllll}1 & 1 & 1 & 1 & 1 & 1 & 1 & 1 & 1 & 1 & 2 & 2 & 2 & 2 & 2 & 2 & 2 & 2 & 2 & 2 & 3 & 3\end{array}$

$\begin{array}{llllllllllllllllllllllllllllllll}0 & 1 & 2 & 3 & 4 & 5 & 6 & 7 & 8 & 9 & 0 & 1 & 2 & 3 & 4 & 5 & 6 & 7 & 8 & 9 & 0 & 1 & 2 & 3 & 4 & 5 & 6 & 7 & 8 & 9 & 0 & 1\end{array}$

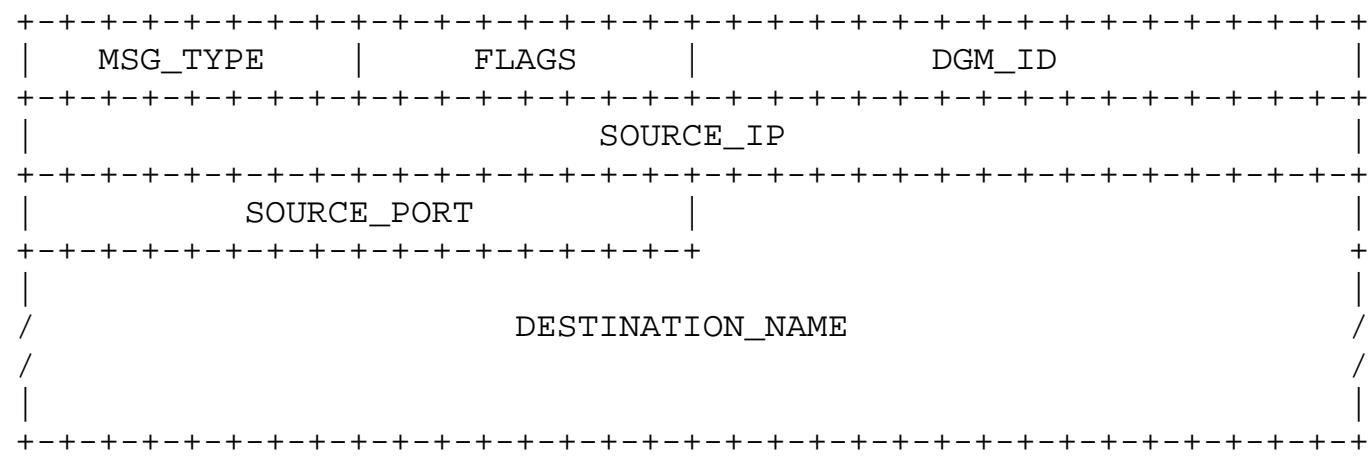




\section{PROTOCOL DESCRIPTIONS}

\subsection{NAME SERVICE PROTOCOLS}

A REQUEST packet is always sent to the well known UDP port NAME_SERVICE_UDP_PORT. The destination address is normally either the IP broadcast address or the address of the NBNS - the address of the NBNS server it set up at initialization time. In rare cases, a request packet will be sent to an end node, e.g. a NAME QUERY REQUEST sent to "challenge" a node.

A RESPONSE packet is always sent to the source UDP port and source IP address of the request packet.

A DEMAND packet must always be sent to the well known UDP port NAME_SERVICE_UDP_PORT. There is no restriction on the target IP address.

Terms used in this section:

tid - Transaction ID. This is a value composed from the requestor's IP address and a unique 16 bit value generated by the originator of the transaction.

\subsubsection{B-NODE ACTIVITY}

5.1.1.1. B-NODE ADD NAME

PROCEDURE add_name (newname)

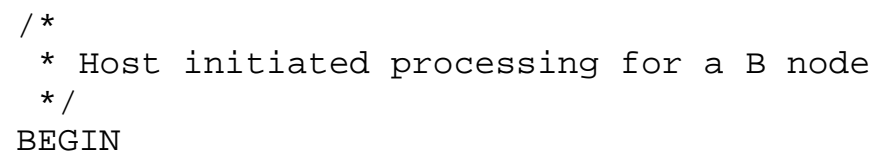

REPEAT

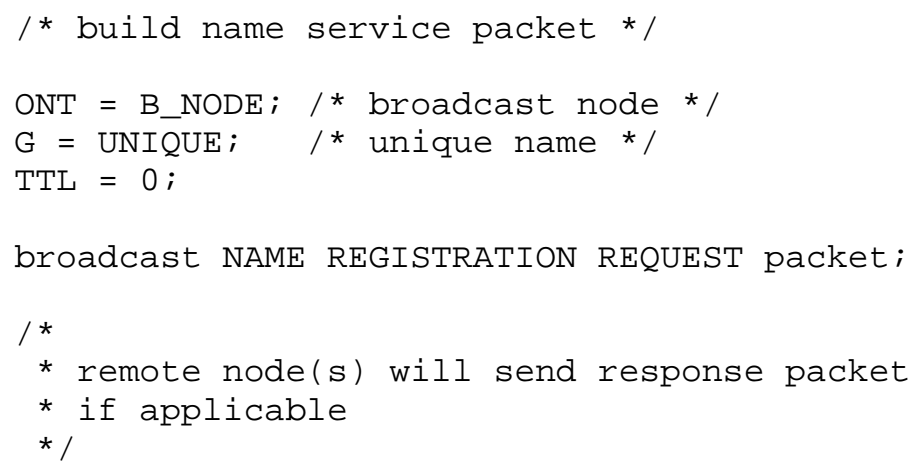




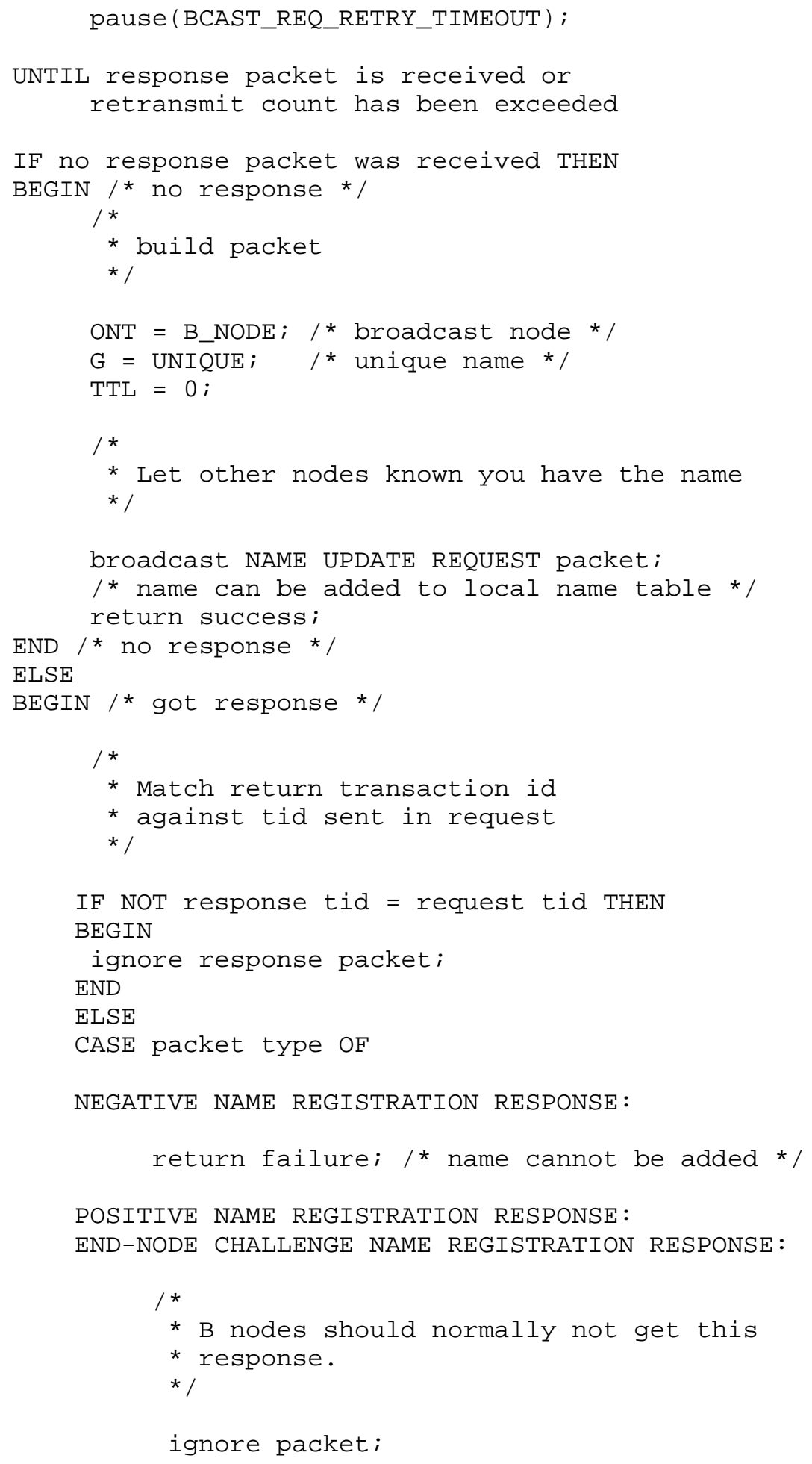




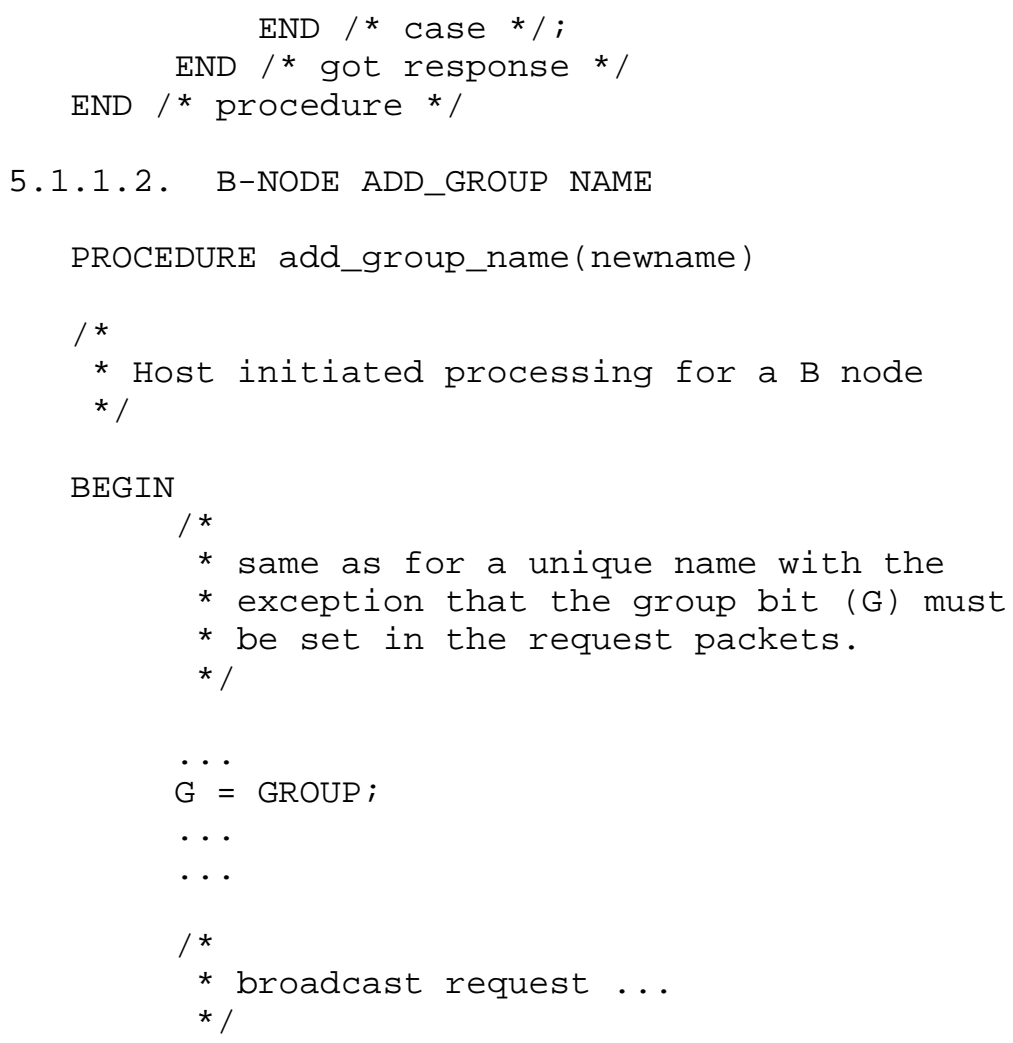




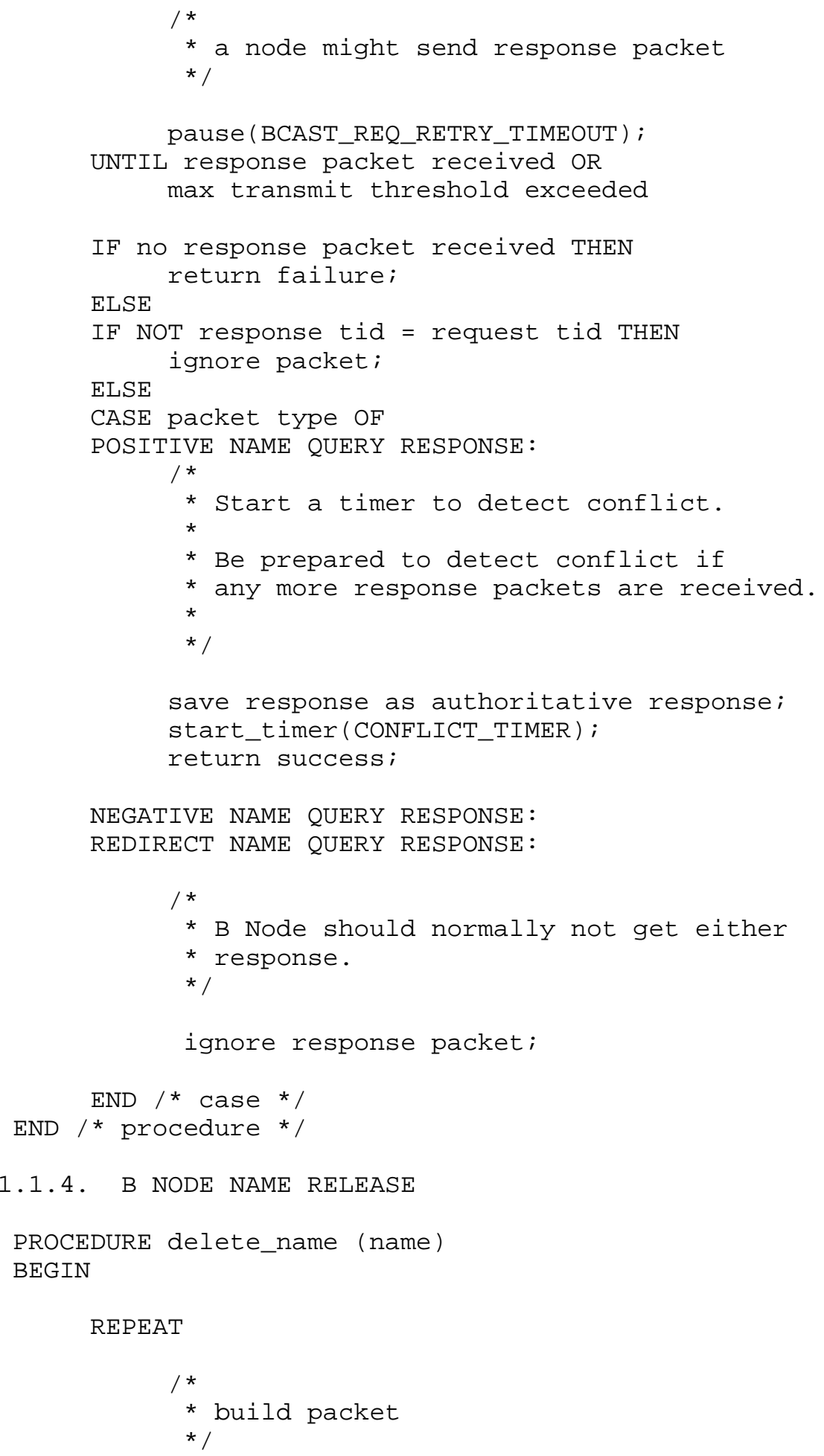




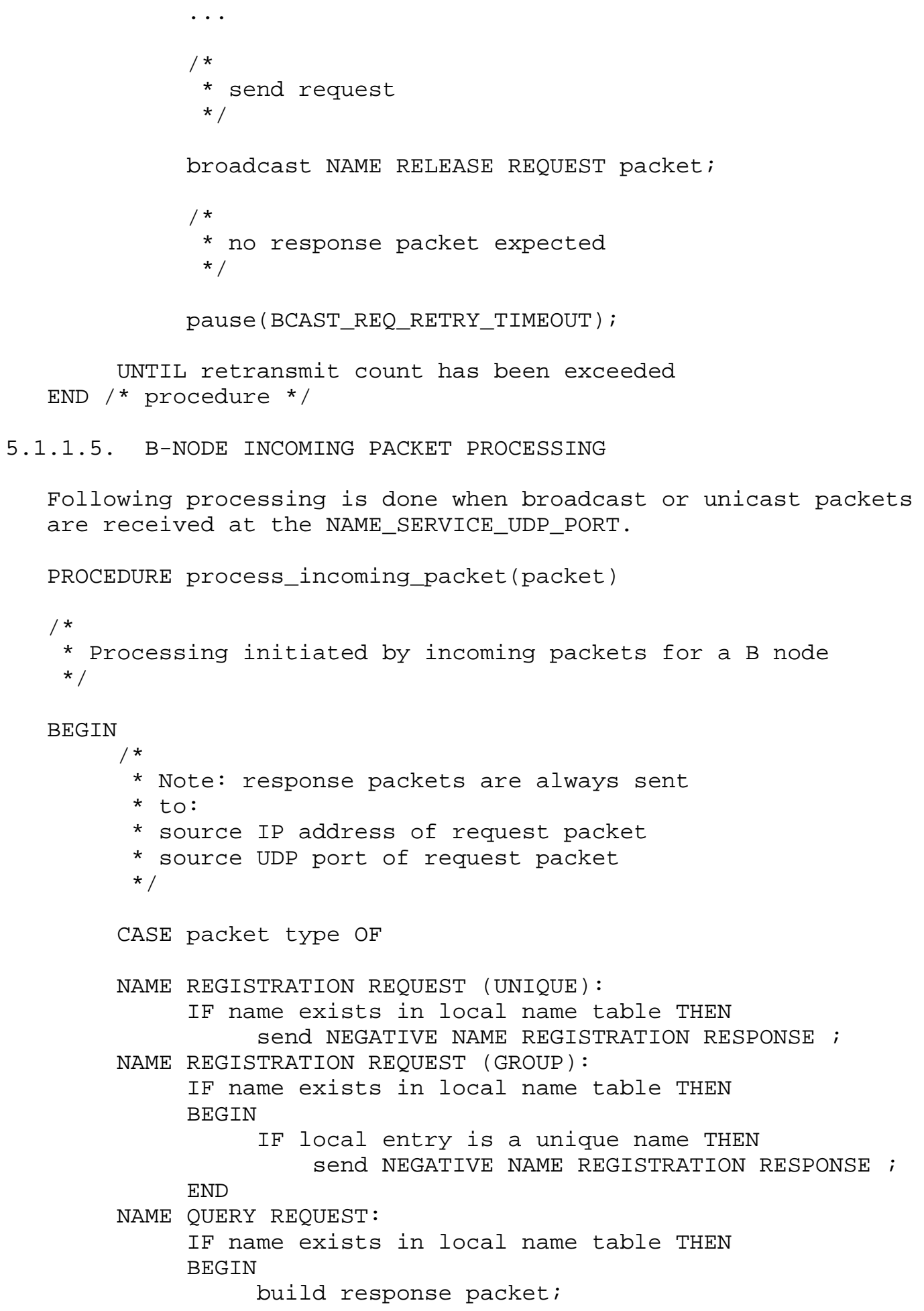




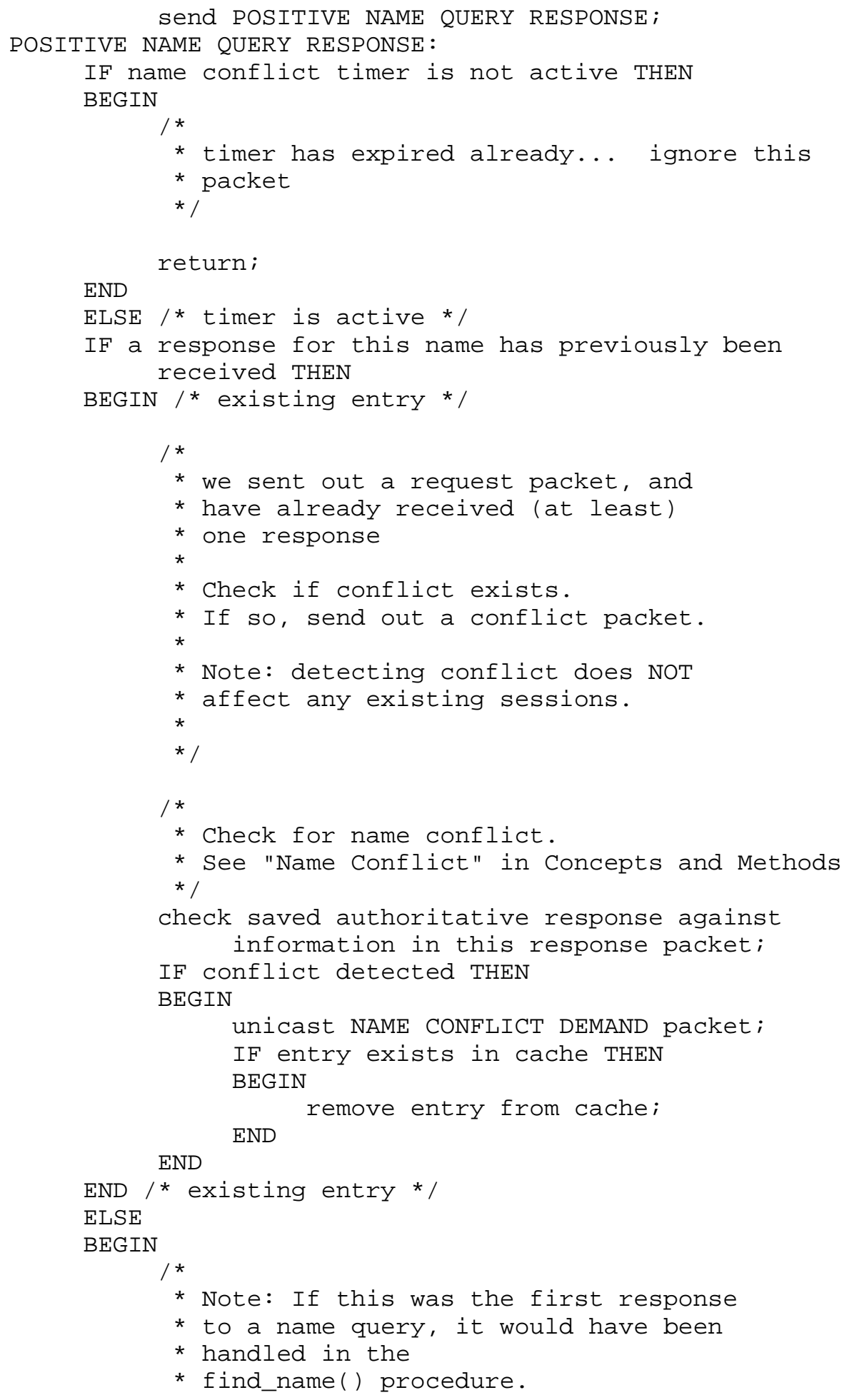




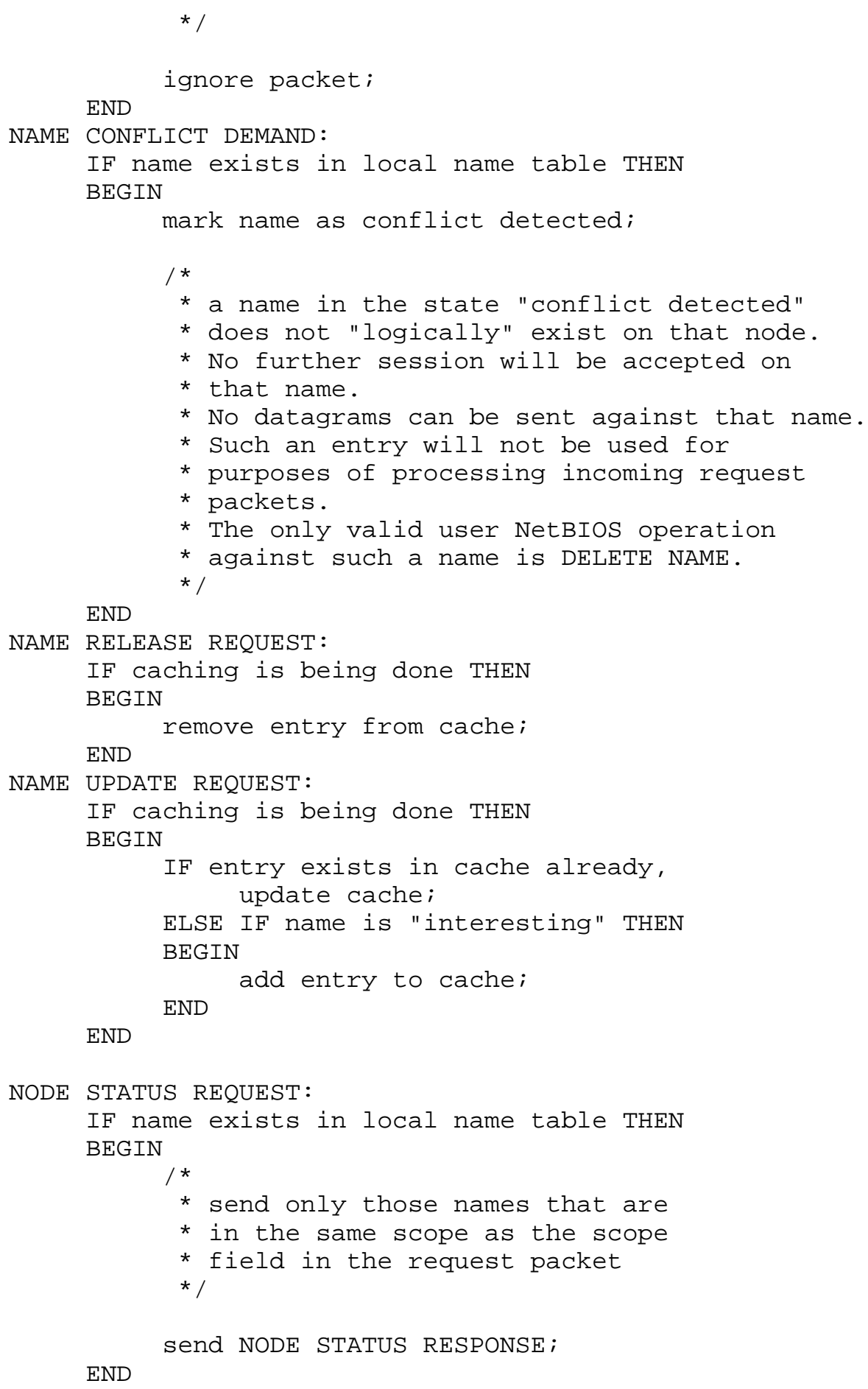




\subsubsection{P-NODE ACTIVITY}

All packets sent or received by $P$ nodes are unicast UDP packets. A $P$ node sends name service requests to the NBNS node that is specified in the P-node configuration.

\subsubsection{P-NODE ADD_NAME}

PROCEDURE add_name (newname)

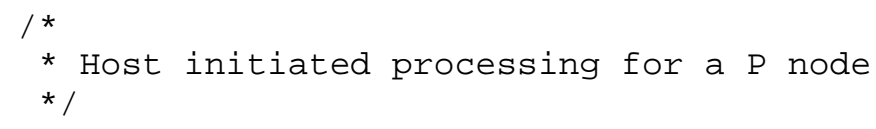

BEGIN

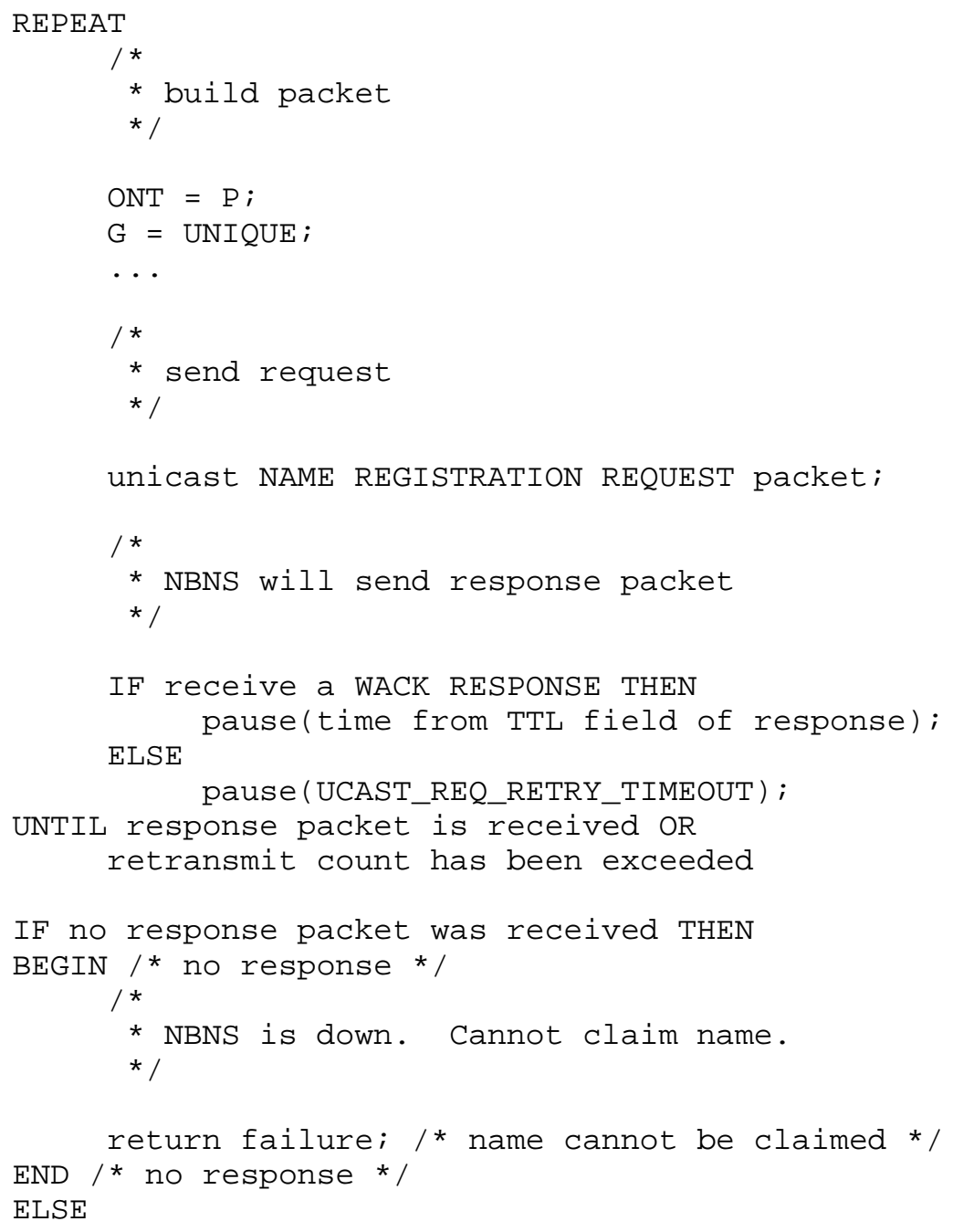




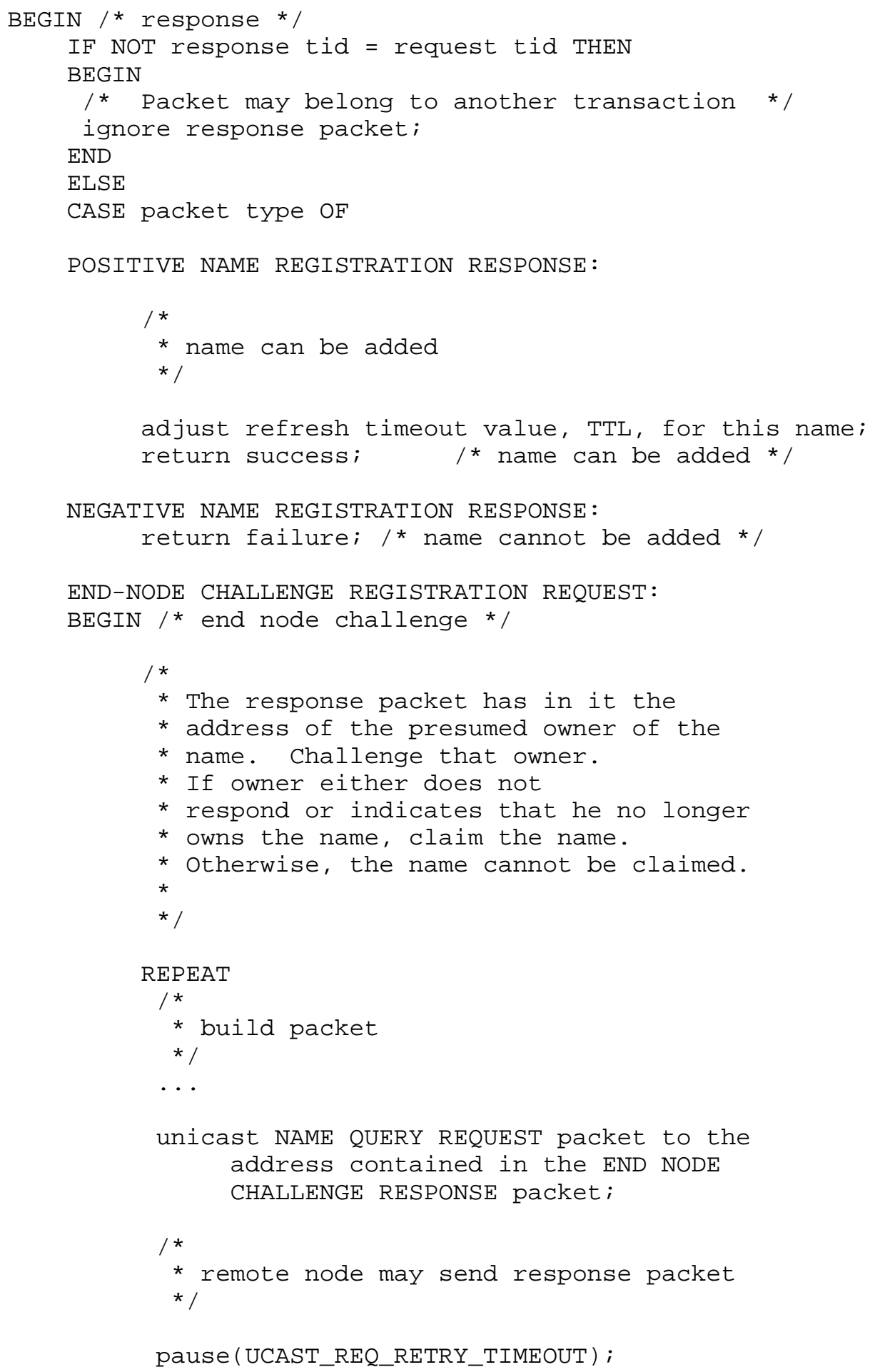




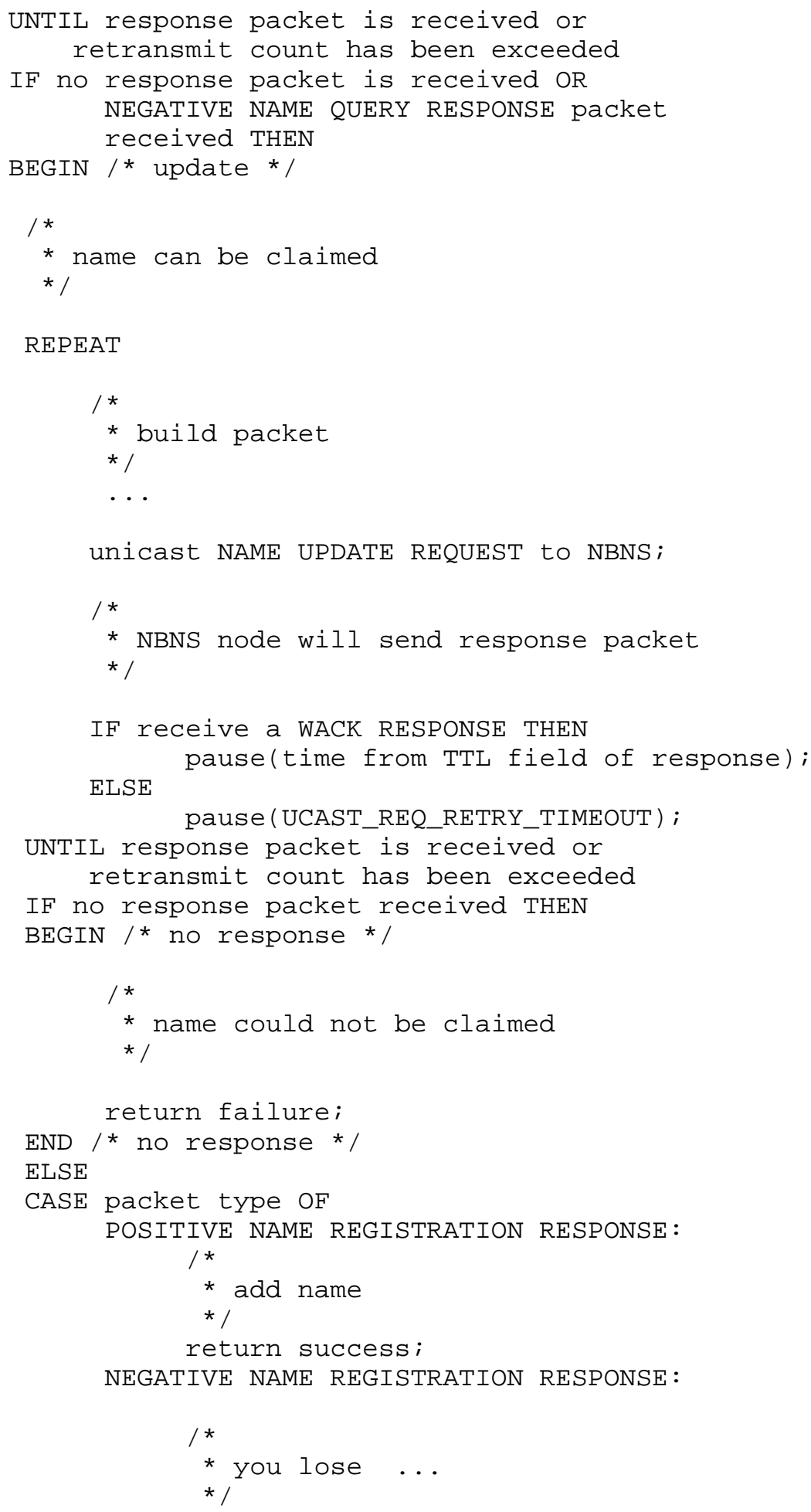




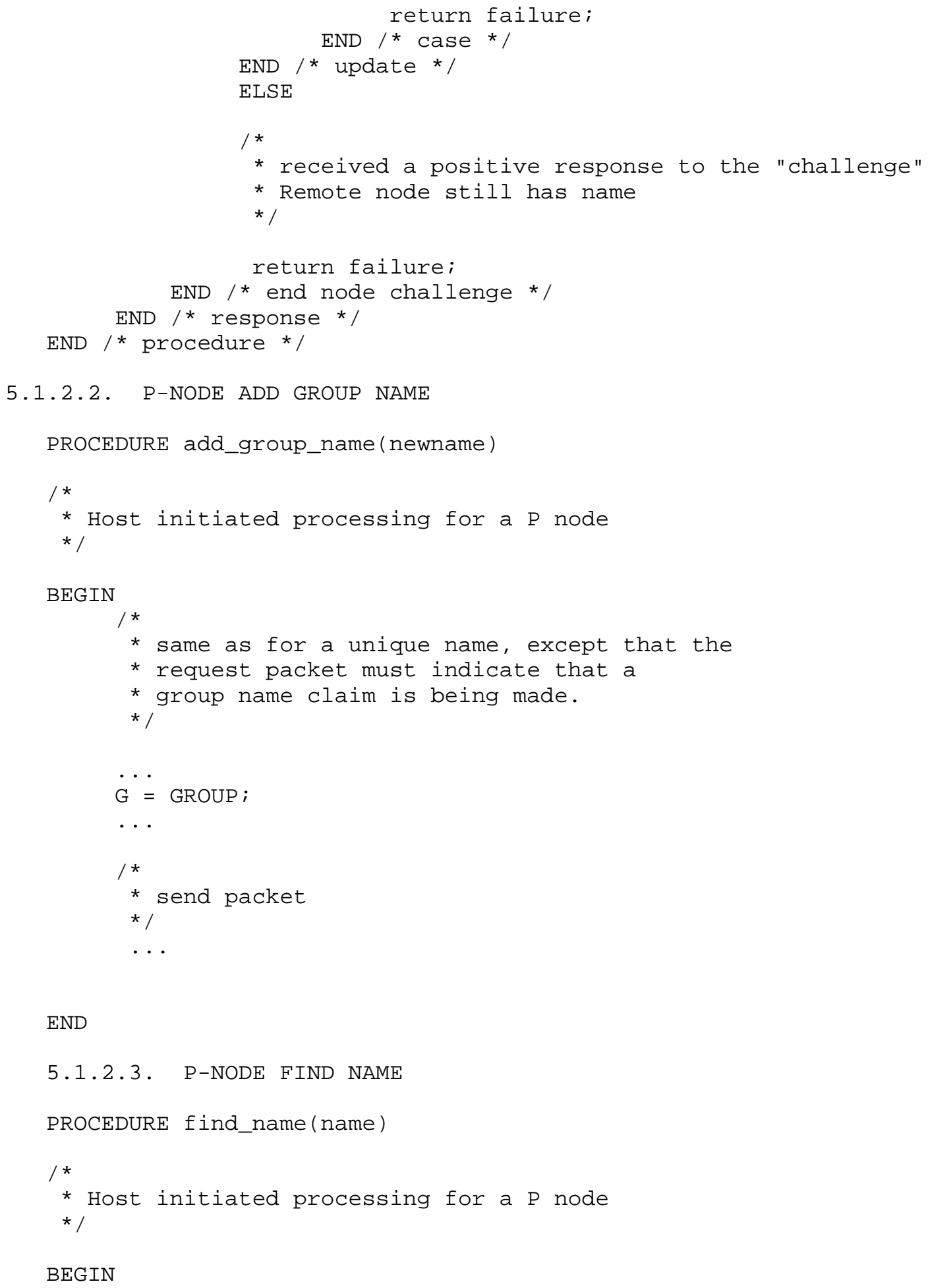

END

\subsubsection{P-NODE FIND NAME}




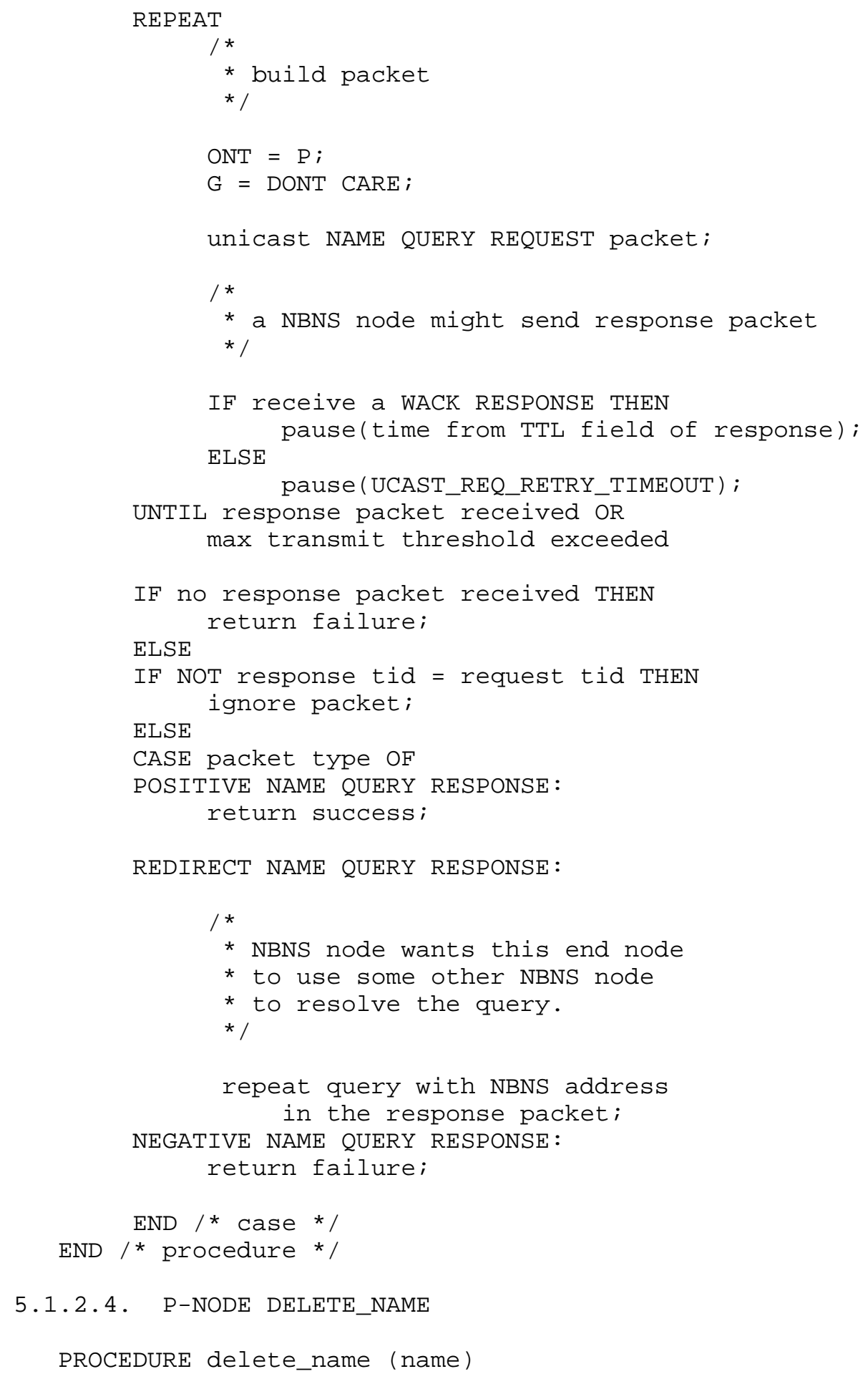




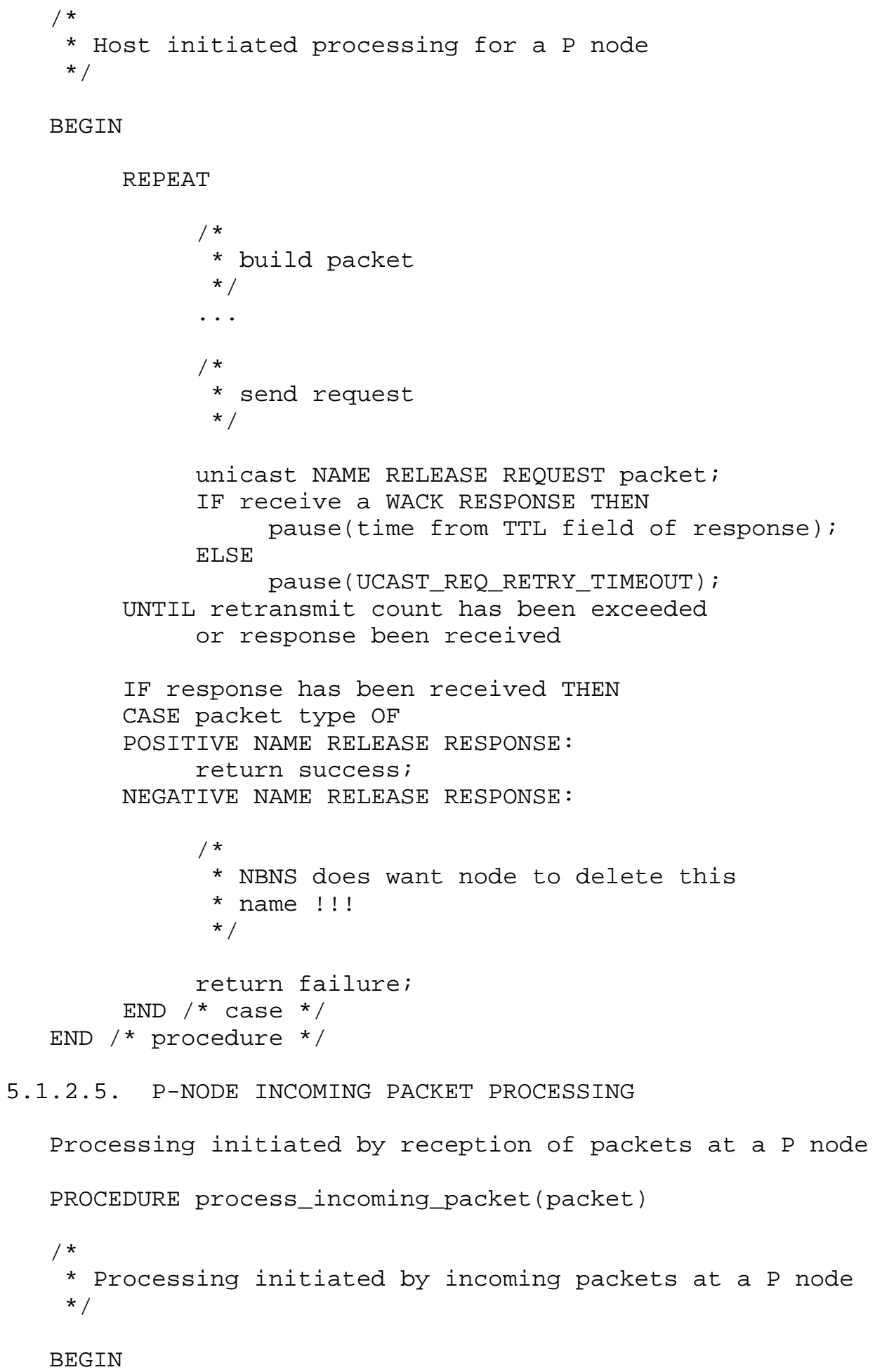




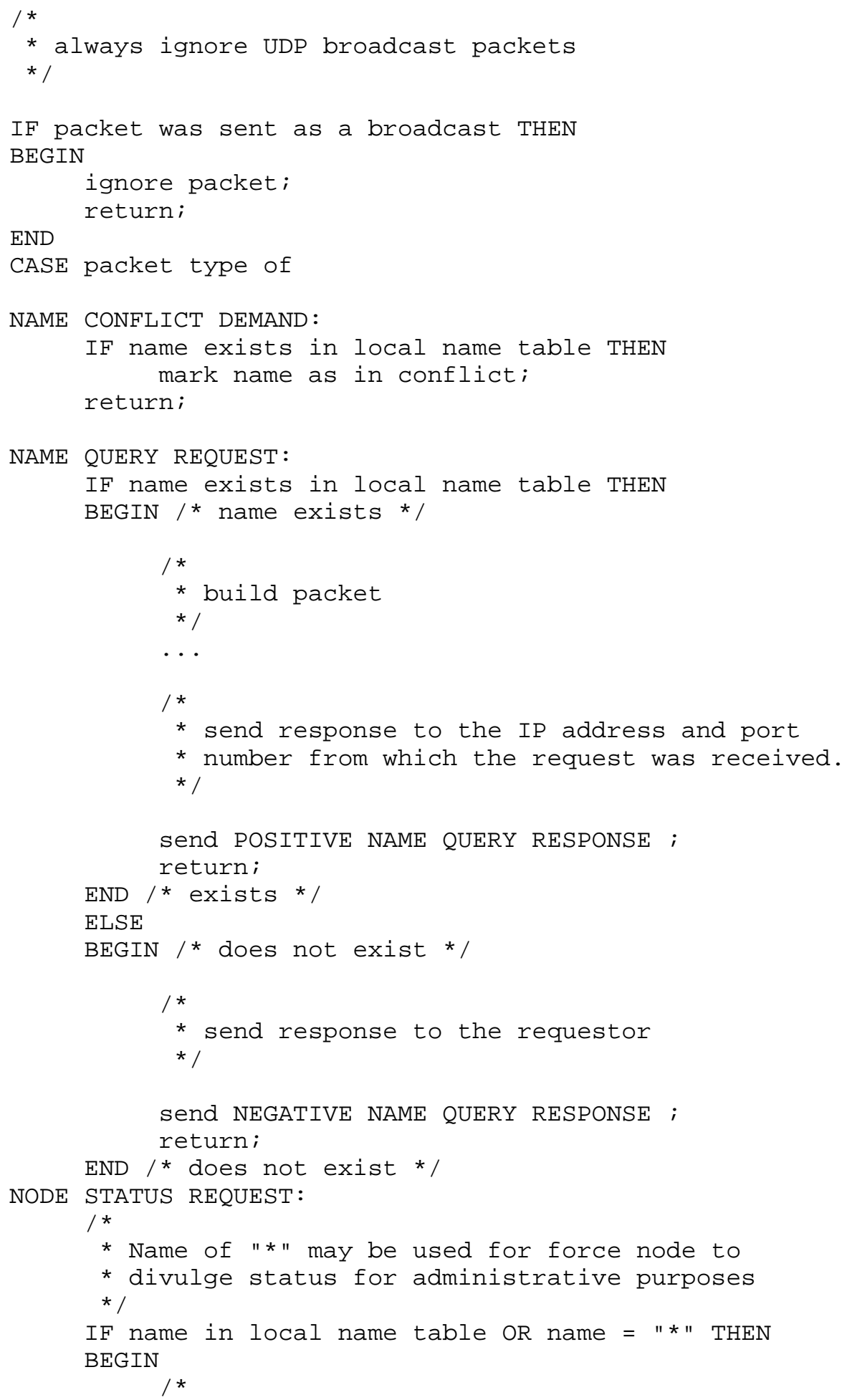




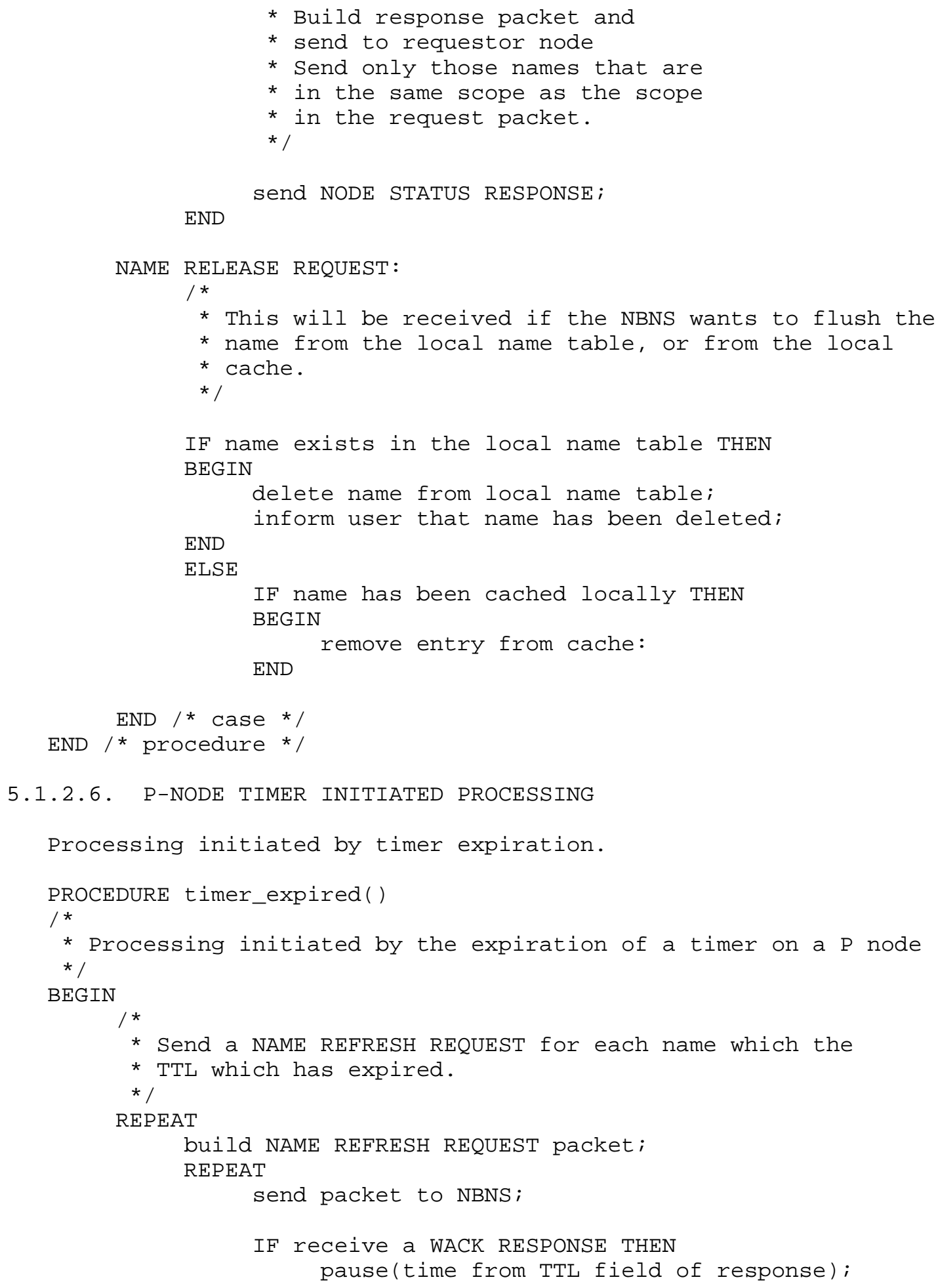




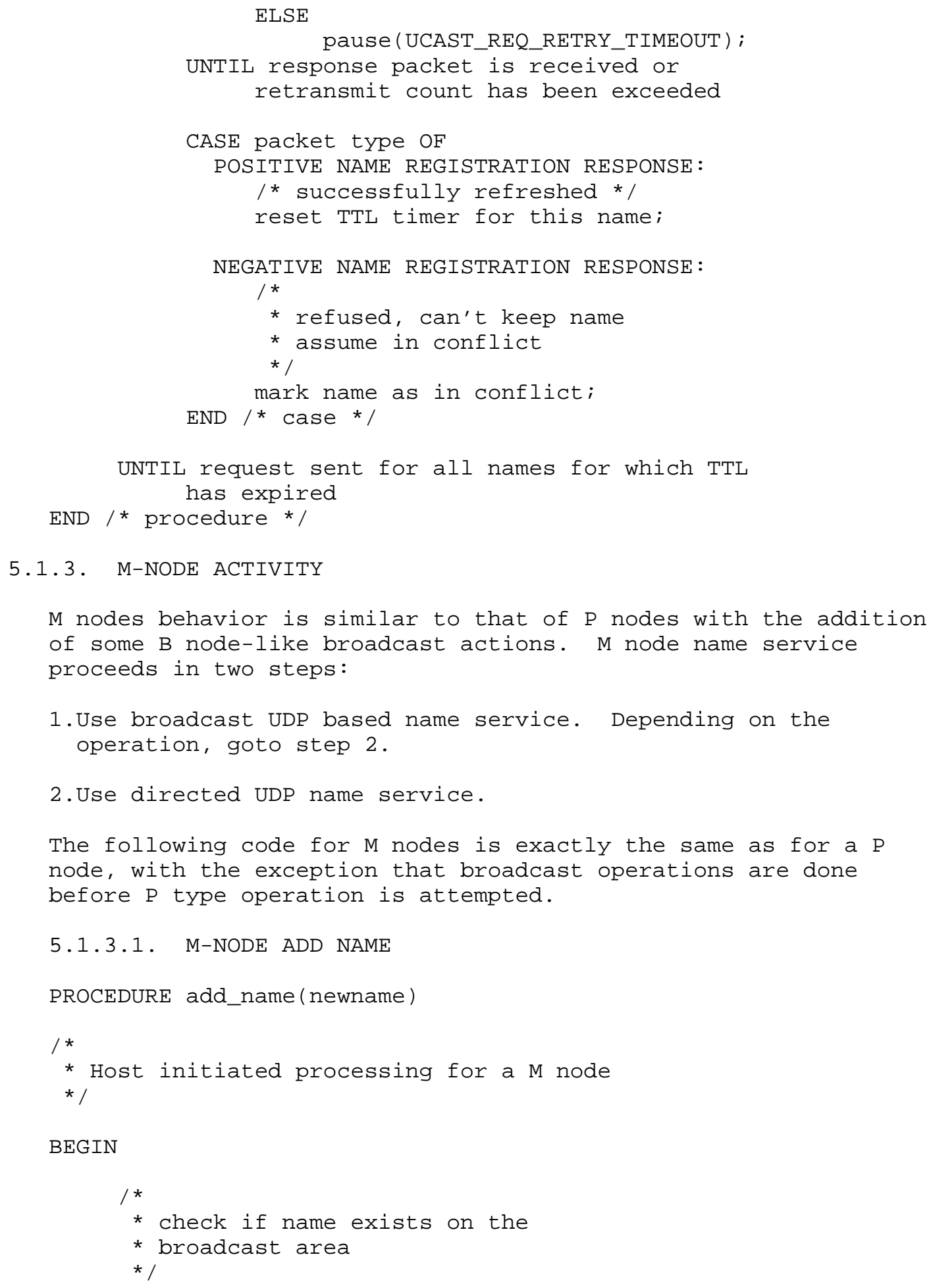




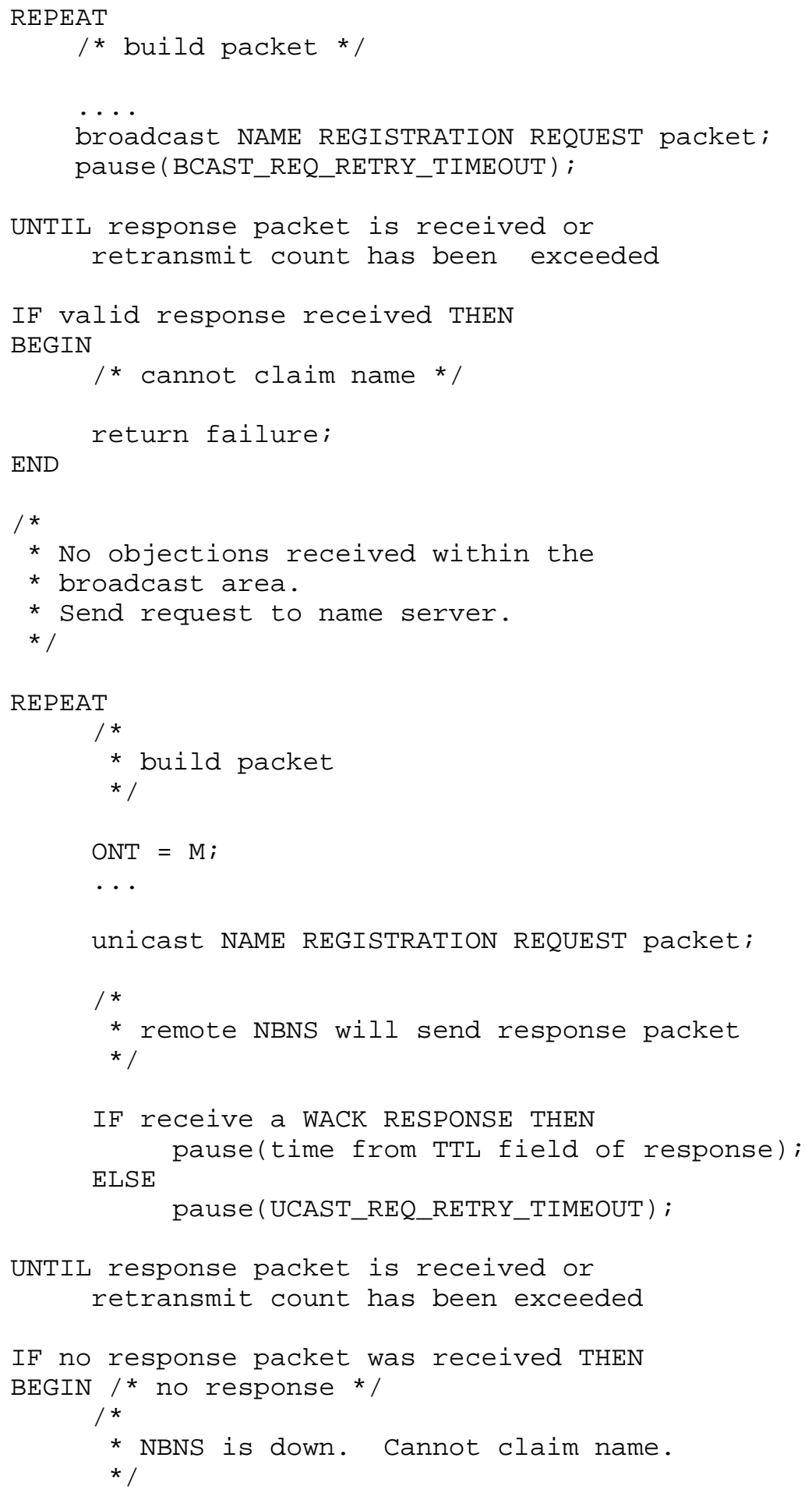




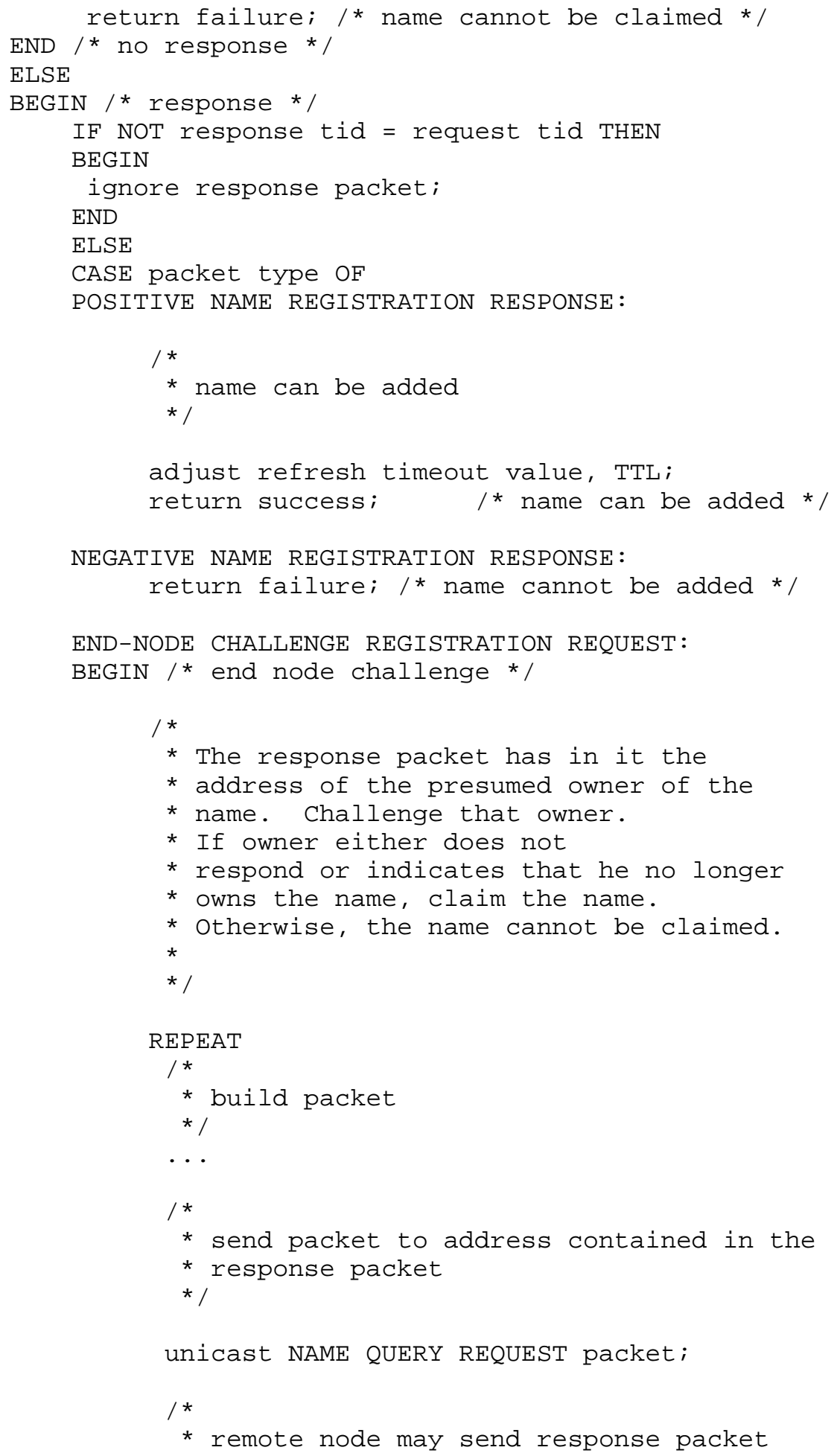




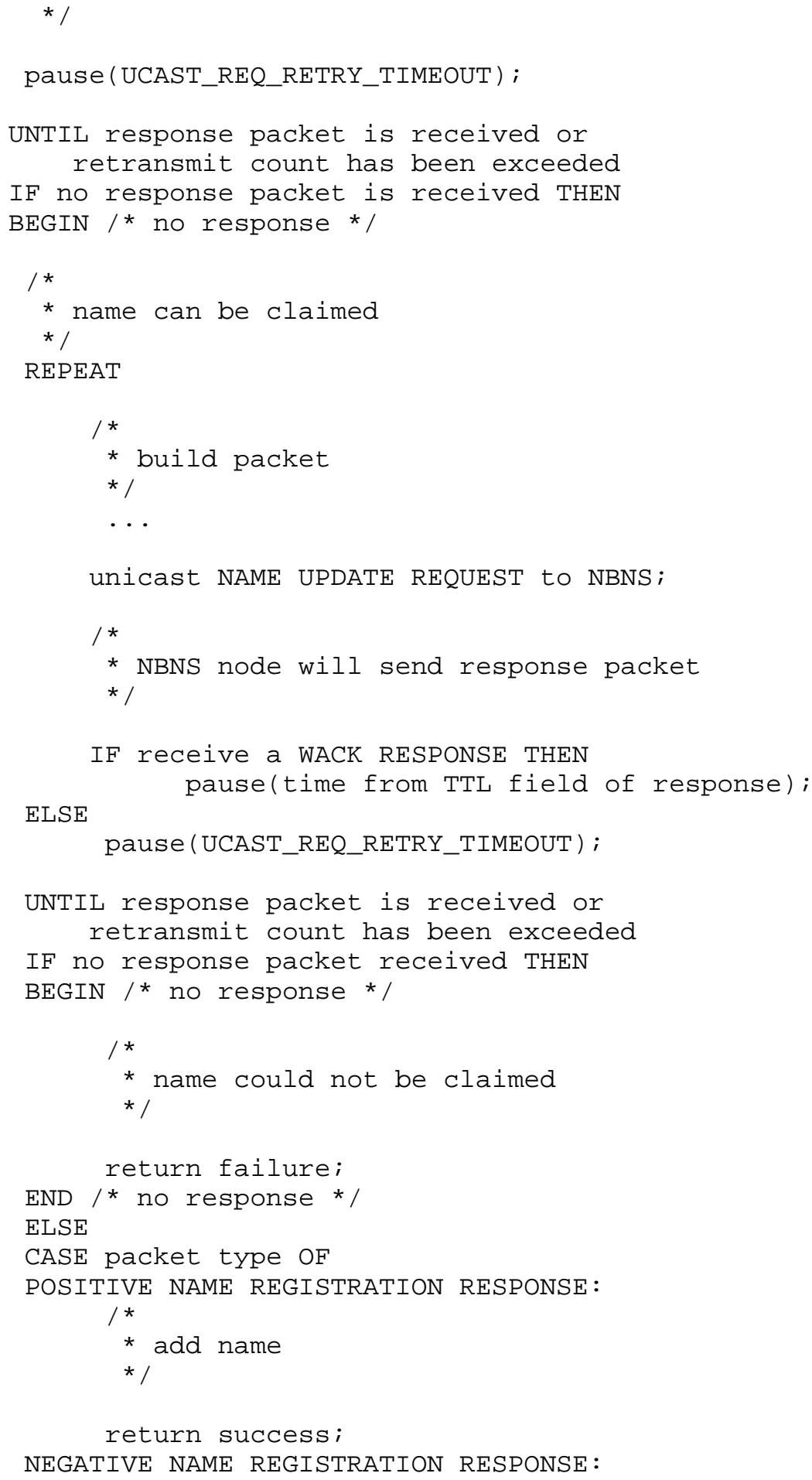




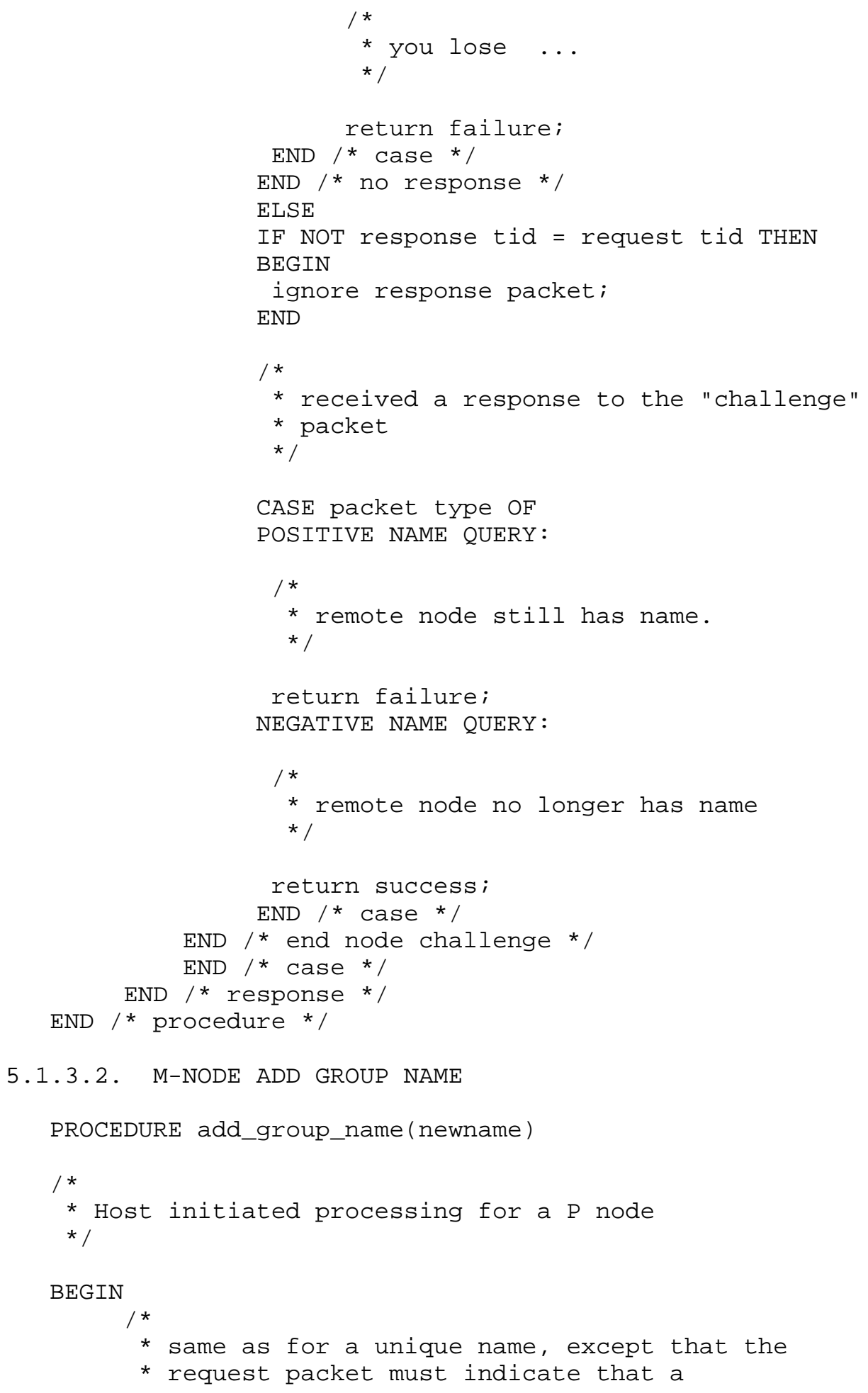




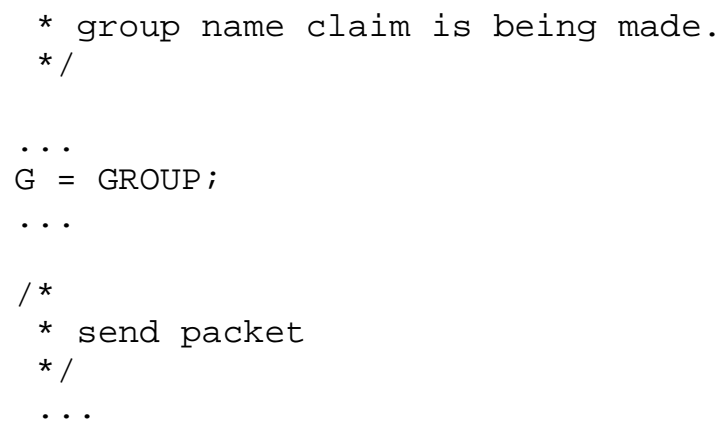

END

5.1.3.3. M-NODE FIND NAME

PROCEDURE find_name (name)

/*

* Host initiated processing for a M node

*

BEGIN

$/ \star$

* check if any node on the broadcast

* area has the name

$\star /$

REPEAT

/* build packet */

...

broadcast NAME QUERY REQUEST packet;

pause (BCAST_REQ_RETRY_TIMEOUT) ;

UNTIL response packet received OR

max transmit threshold exceeded

IF valid response received THEN

BEGIN

save response as authoritative response;

start_timer(CONFLICT_TIMER);

return success;

END

$/ \star$

* no valid response on the b'cast segment.

* Try the name server.

* 1

REPEAT

NetBIOS Working Group

[Page 55] 


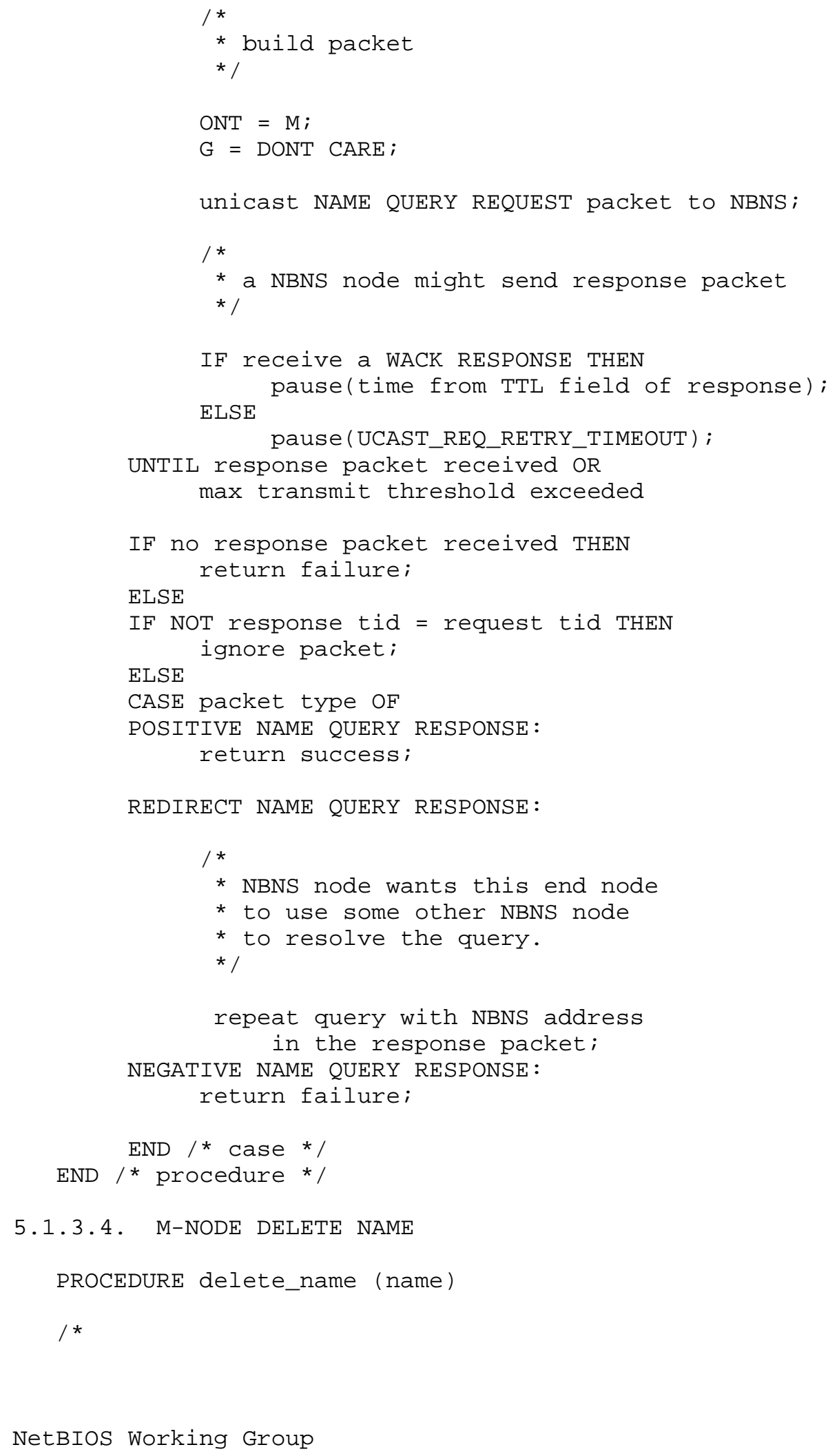


* Host initiated processing for a $\mathrm{P}$ node

$\star /$

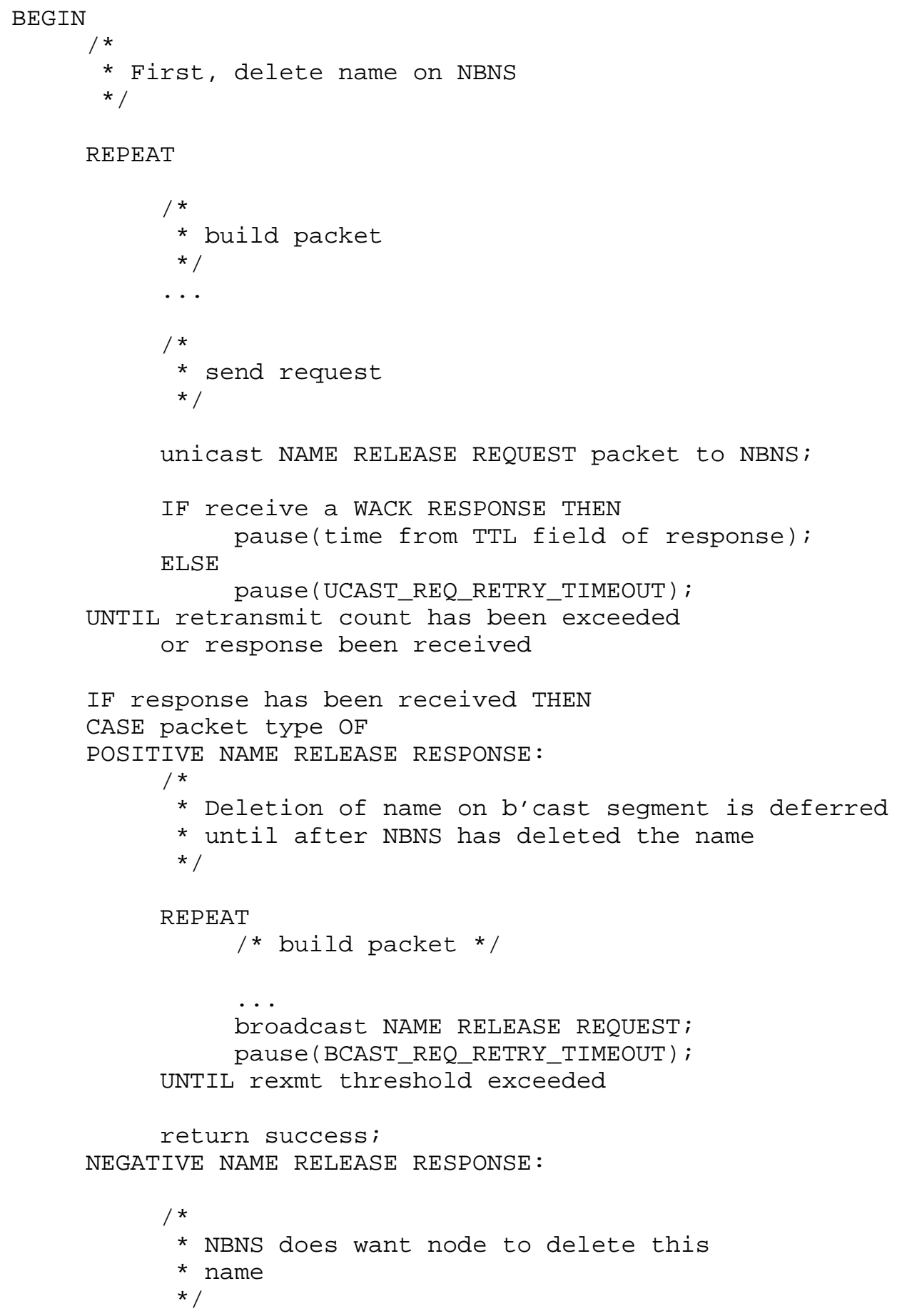




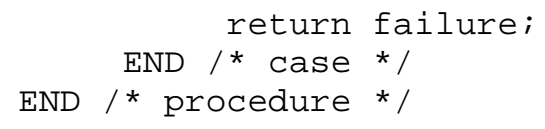

5.1.3.5. M-NODE INCOMING PACKET PROCESSING

Processing initiated by reception of packets at a M node

PROCEDURE process_incoming_packet (packet)

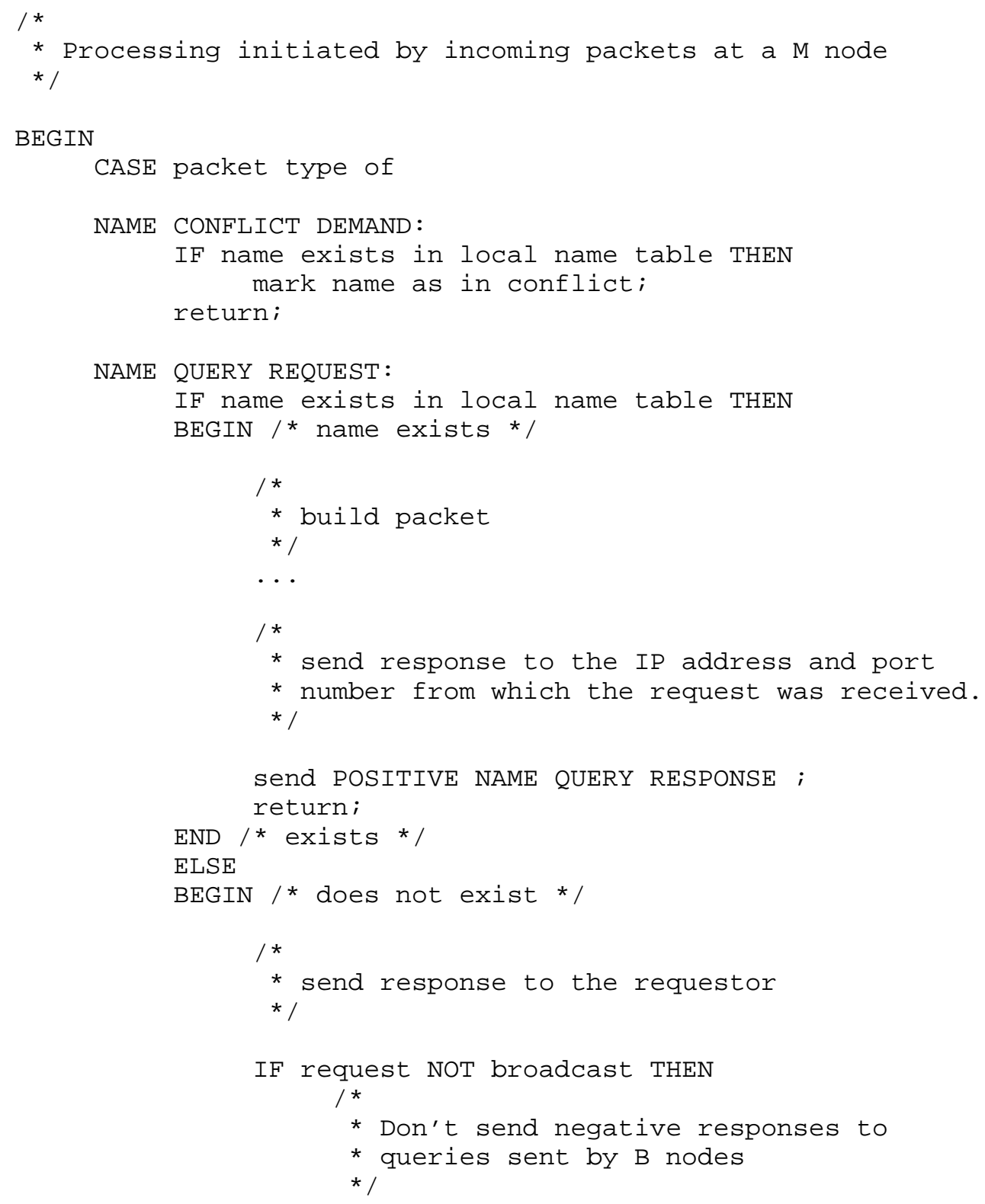




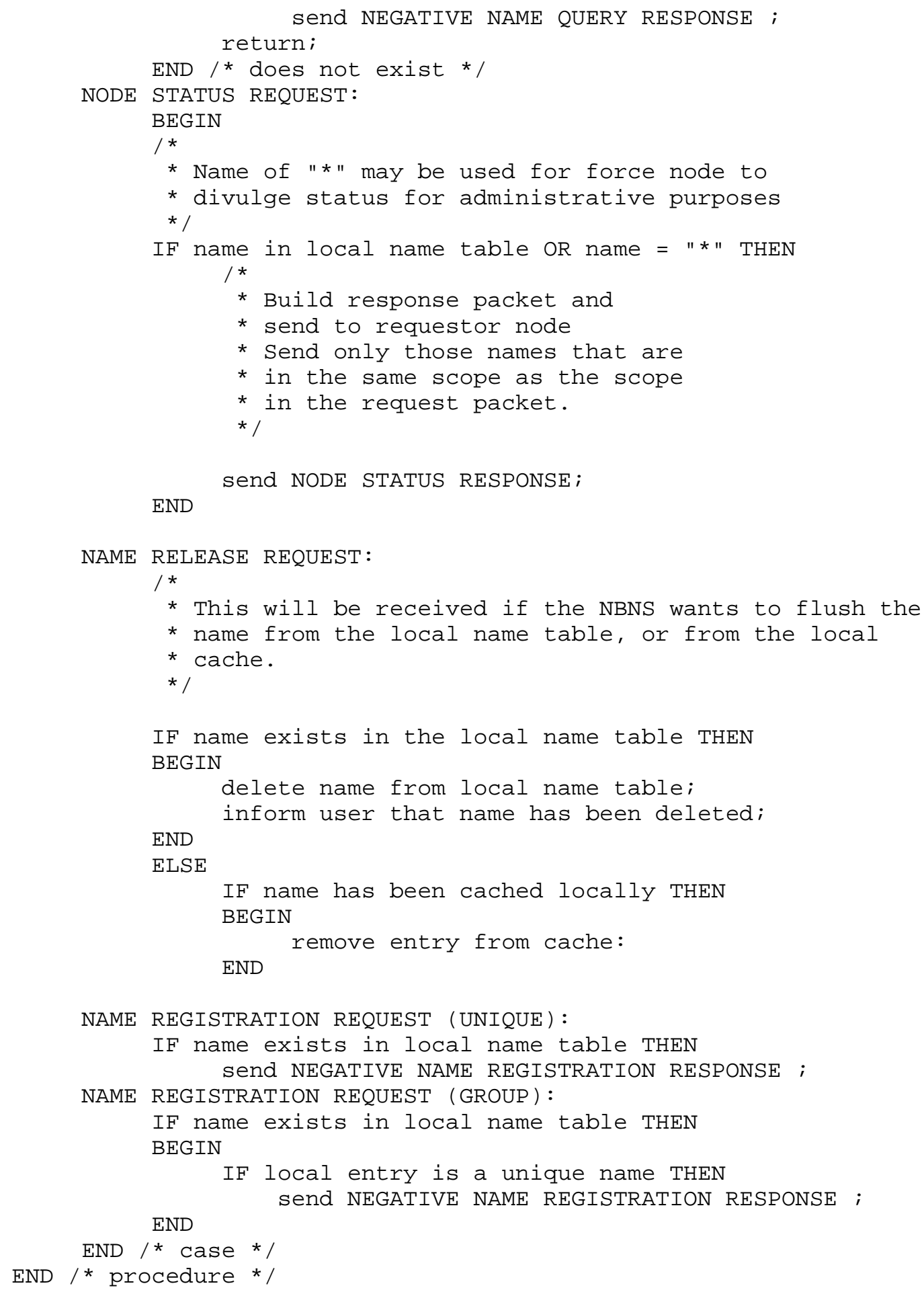




\subsubsection{M-NODE TIMER INITIATED PROCESSING}

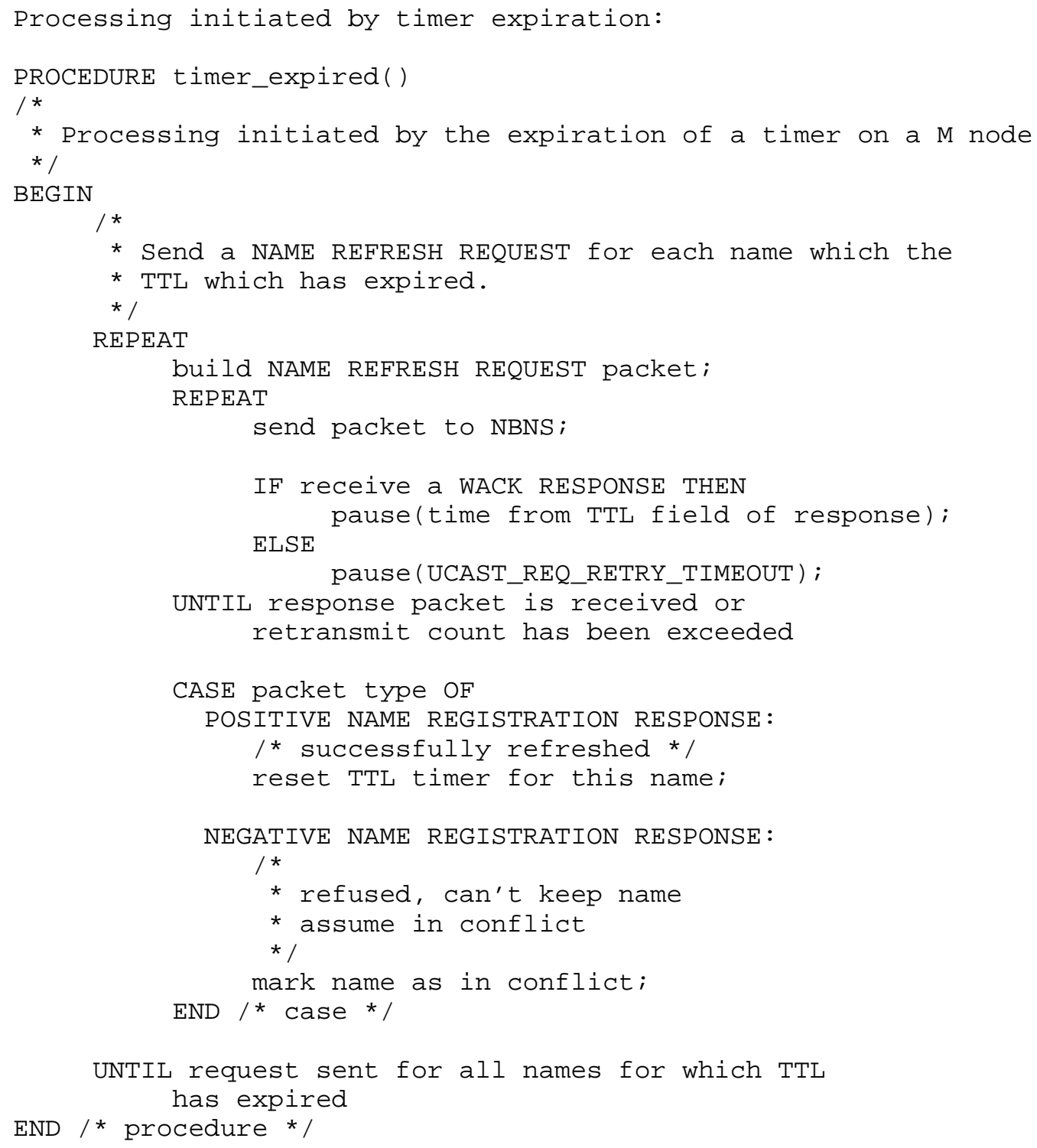

\subsubsection{NBNS ACTIVITY}

A NBNS node will receive directed packets from $\mathrm{P}$ and $\mathrm{M}$ nodes. Reply packets are always sent as directed packets to the source IP address and UDP port number. Received broadcast packets must be ignored. 


\subsubsection{NBNS INCOMING PACKET PROCESSING}

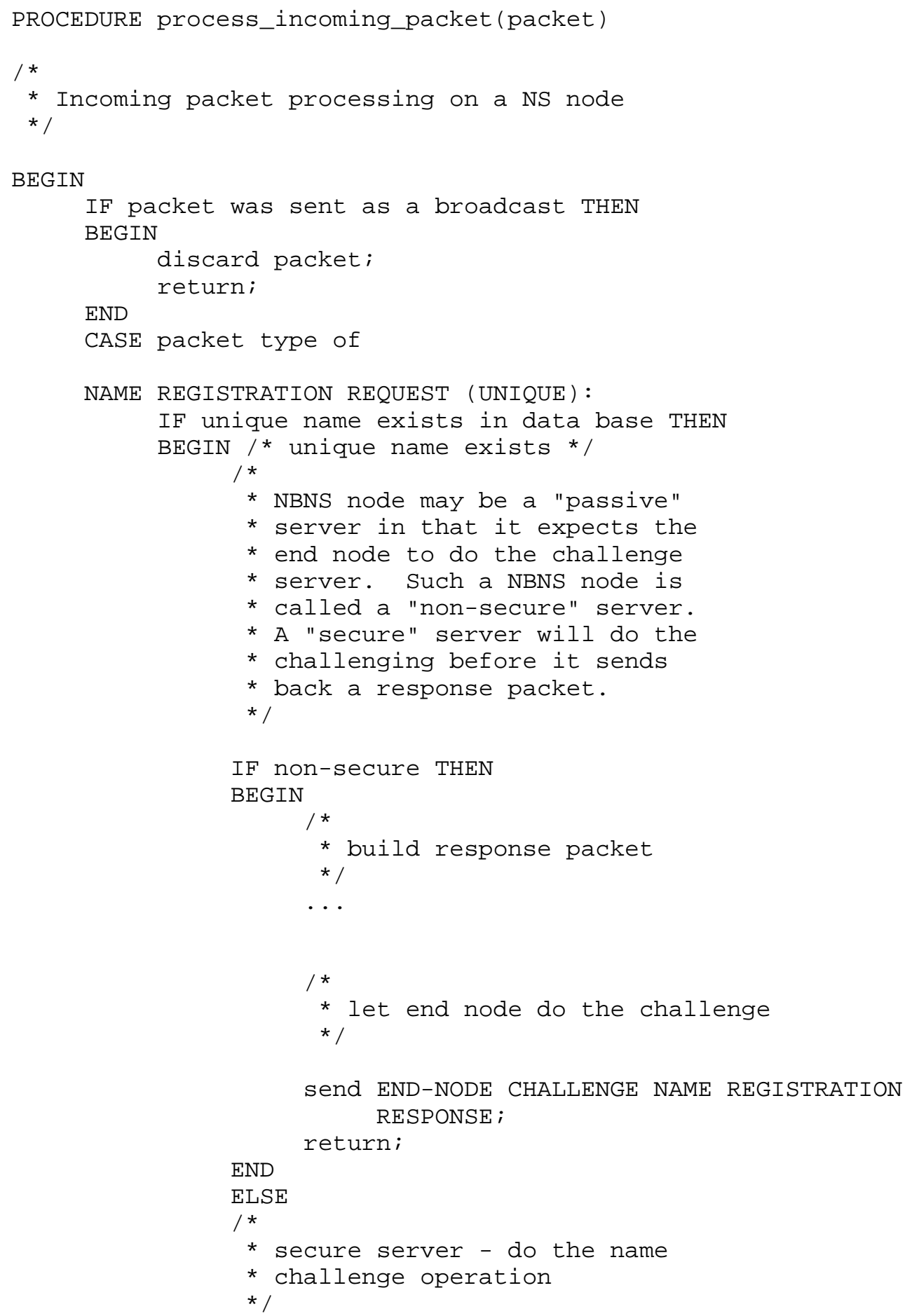




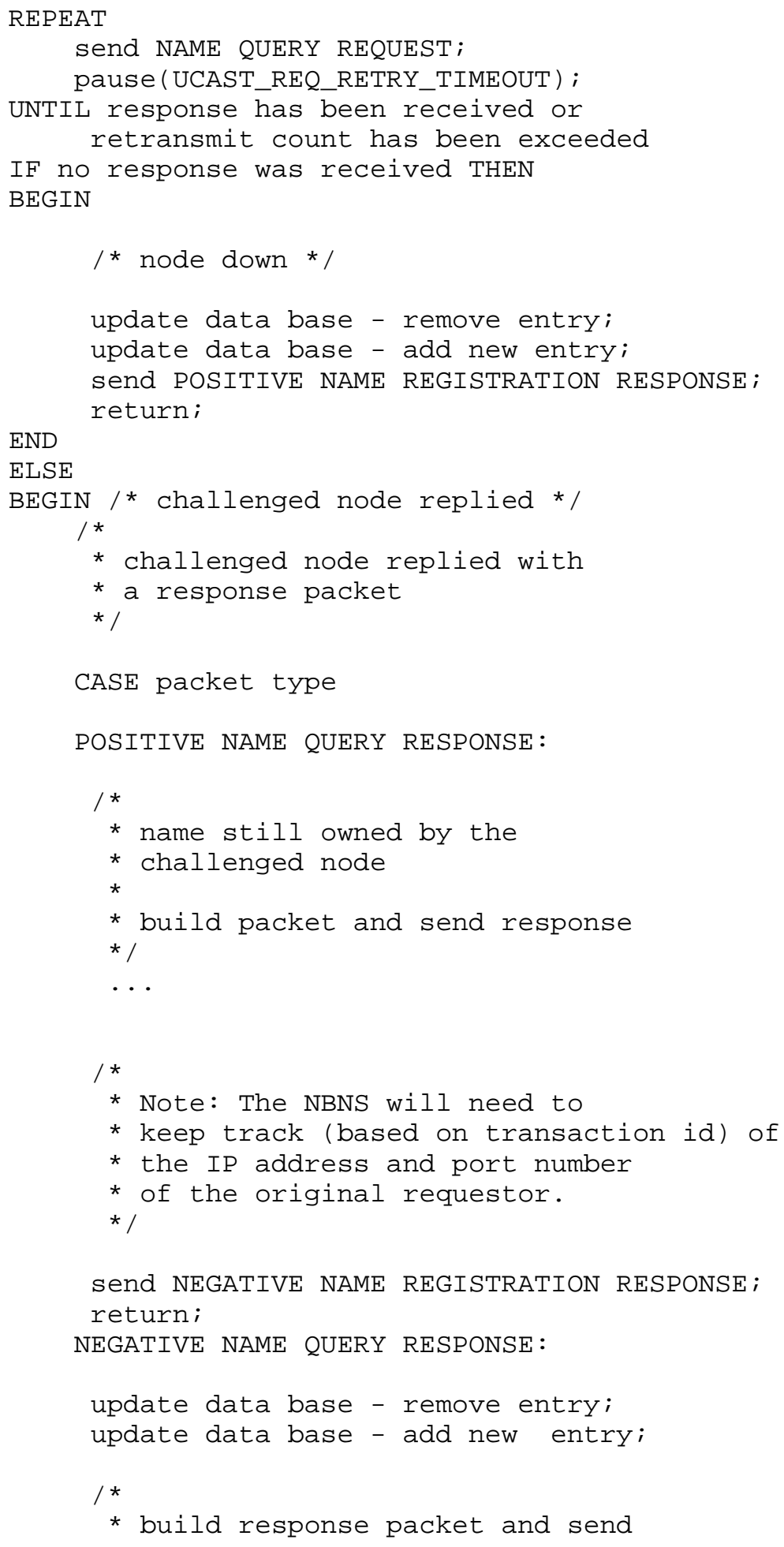




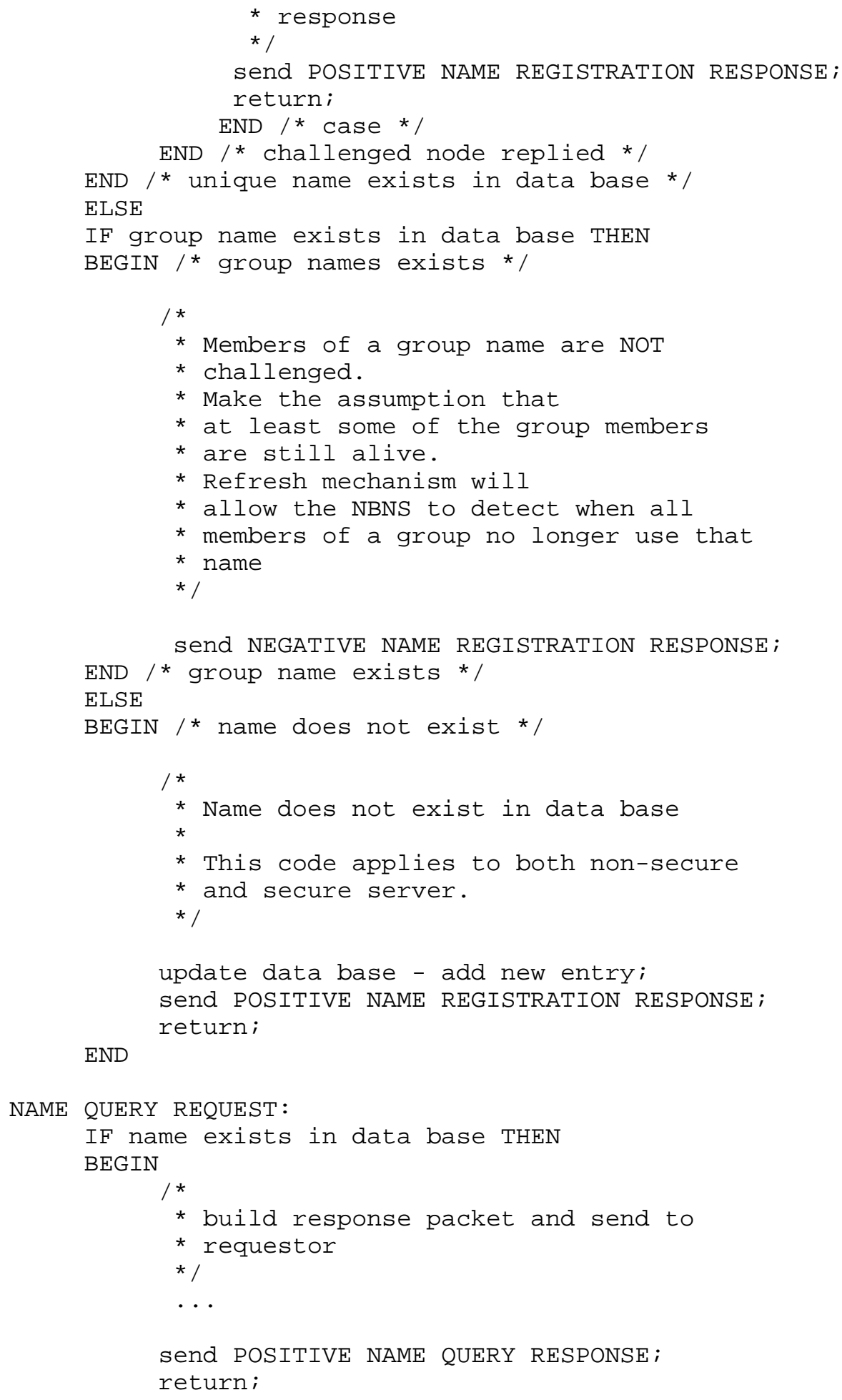




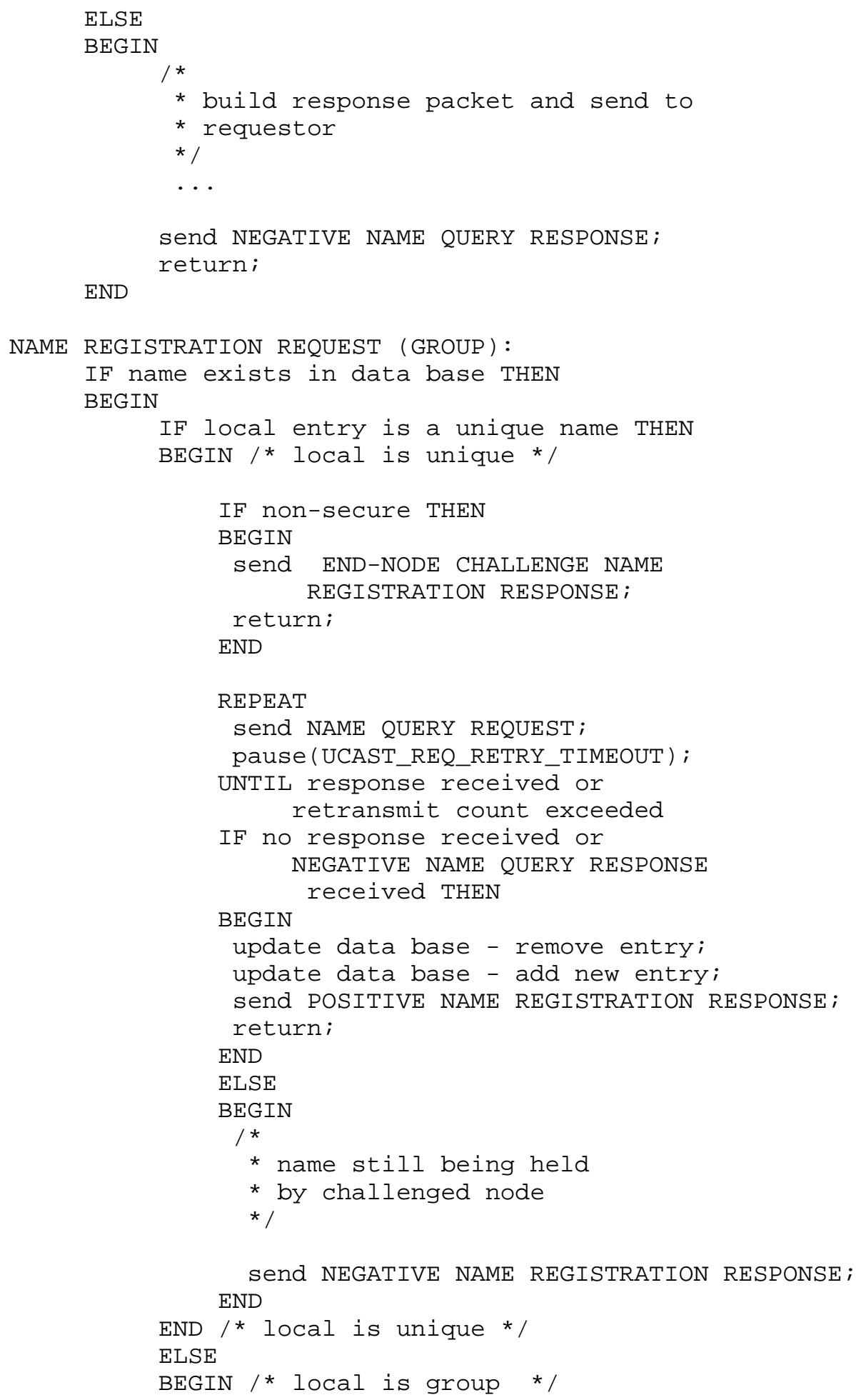




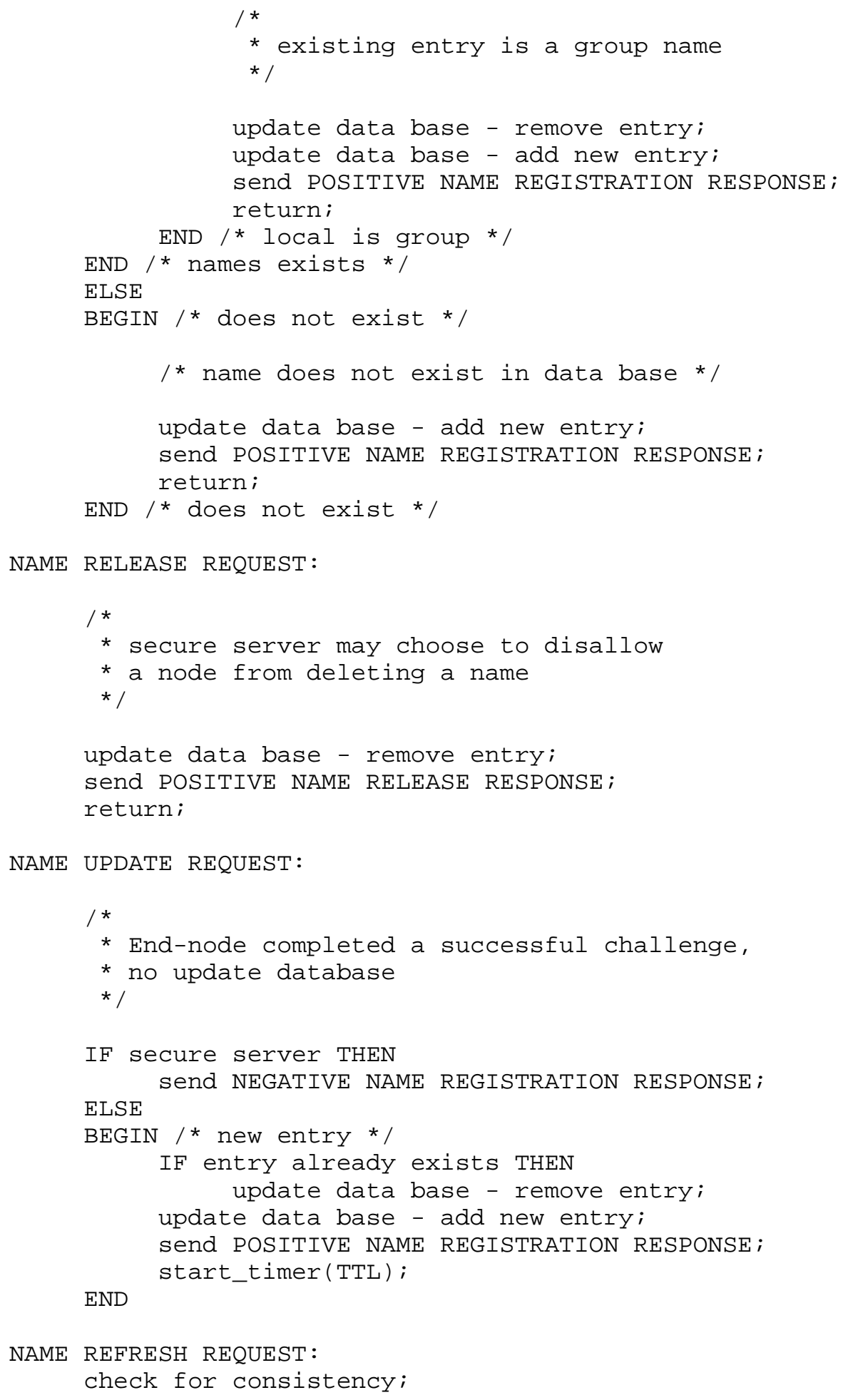




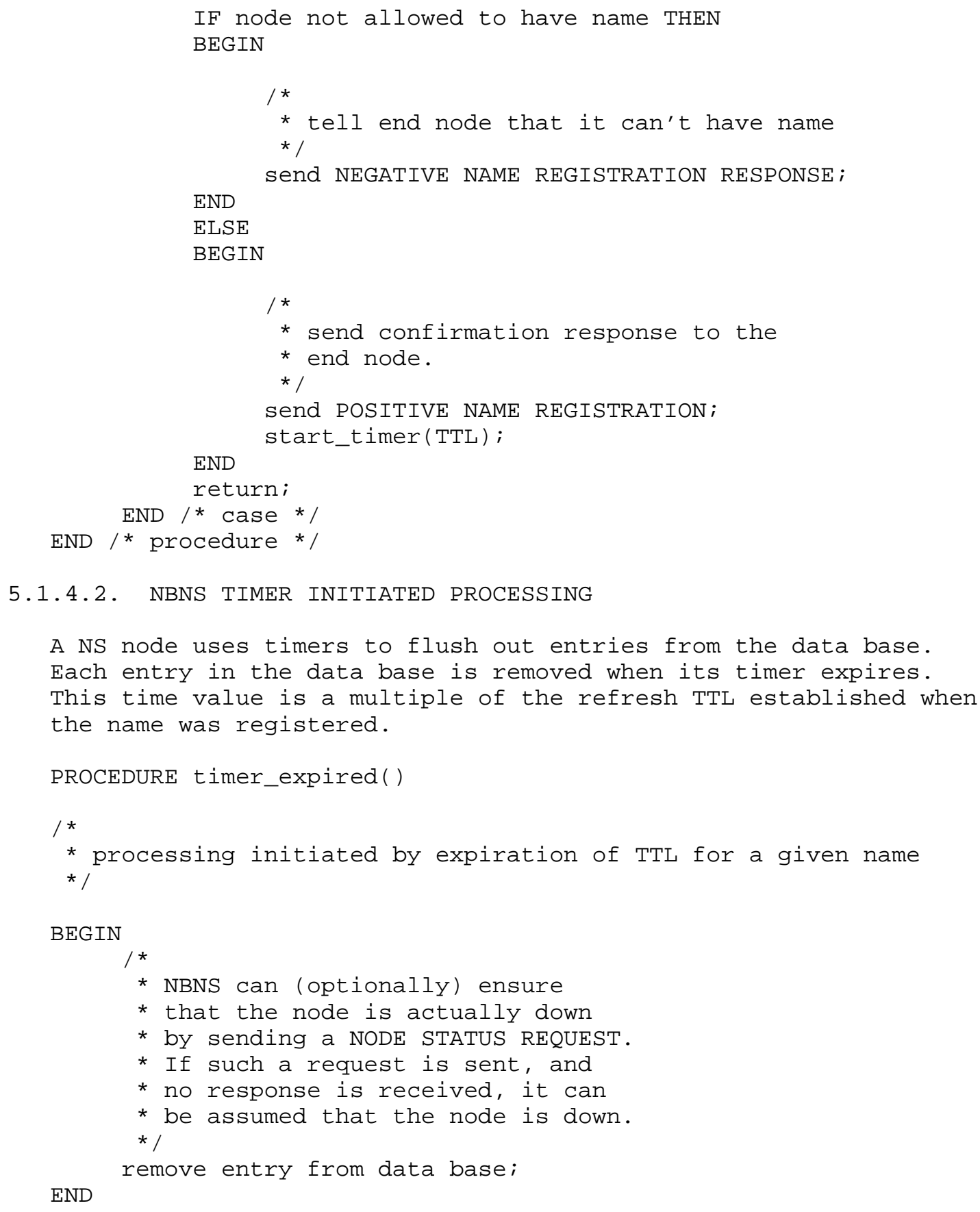

5.1.4.2. NBNS TIMER INITIATED PROCESSING 


\subsection{SESSION SERVICE PROTOCOLS}

The following are variables and should be configurable by the NetBIos user. The default values of these variables is found in "Defined Constants and Variables" in the Detailed Specification.) :

- SSN_RETRY_COUNT - The maximum number TCP connection attempts allowable per a single NetBIOS call request.

- SSN_CLOSE_TIMEOUT is the time period to wait when closing the NetBIOS session before killing the TCP connection if session sends are outstanding.

The following are Defined Constants for the NetBIOS Session Service. (See "Defined Constants and Variables" in the Detailed specification for the value of these constants):

- SSN_SRVC_TCP_PORT - is the globally well-known TCP port allocated for the NetBIOS Session Service. The service accepts TCP connections on this port to establish NetBIOS Sessions. The TCP connection established to this port by the caller is initially used for the exchange of NetBIOS control information. The actual NetBIOS data connection may also pass through this port or, through the retargetting facility, through another port.

\subsubsection{SESSION ESTABLISHMENT PROTOCOLS}

\subsubsection{USER REQUEST PROCESSING}

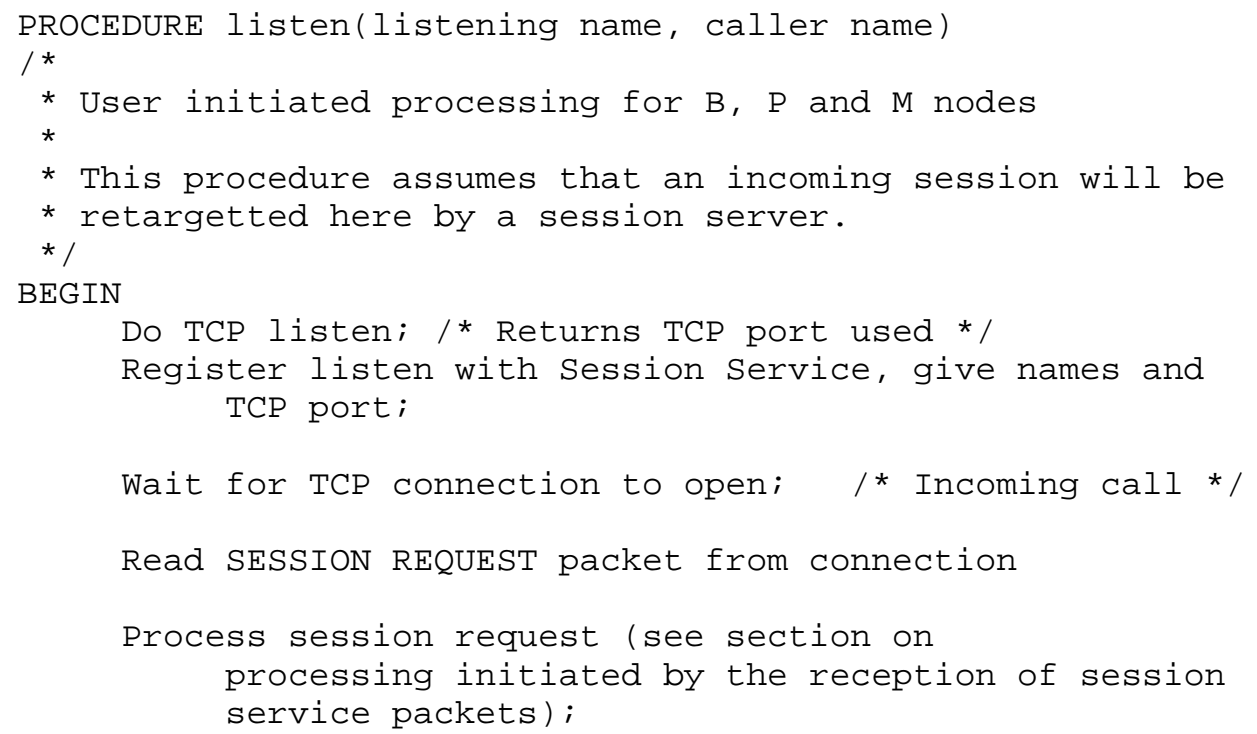




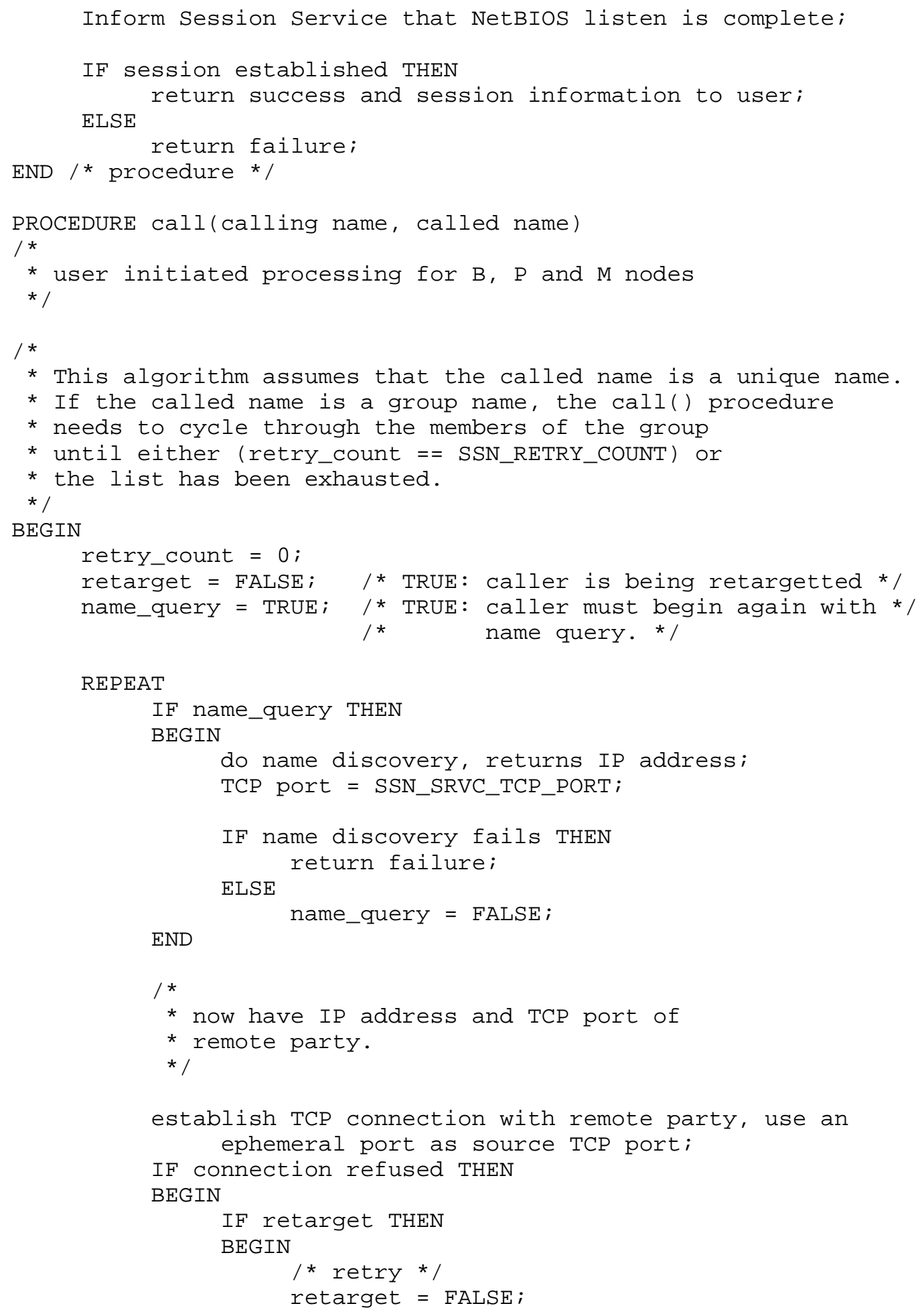




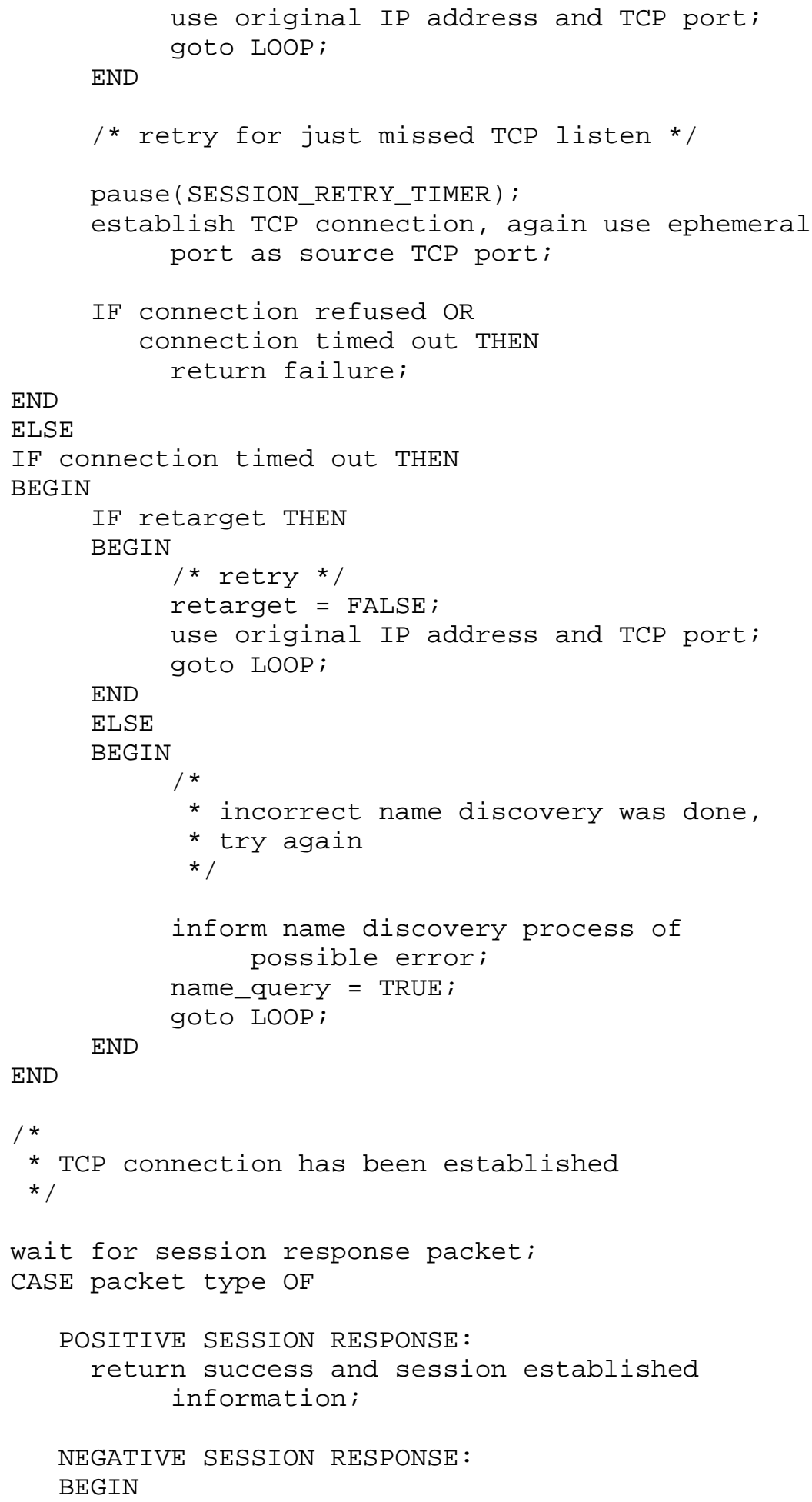




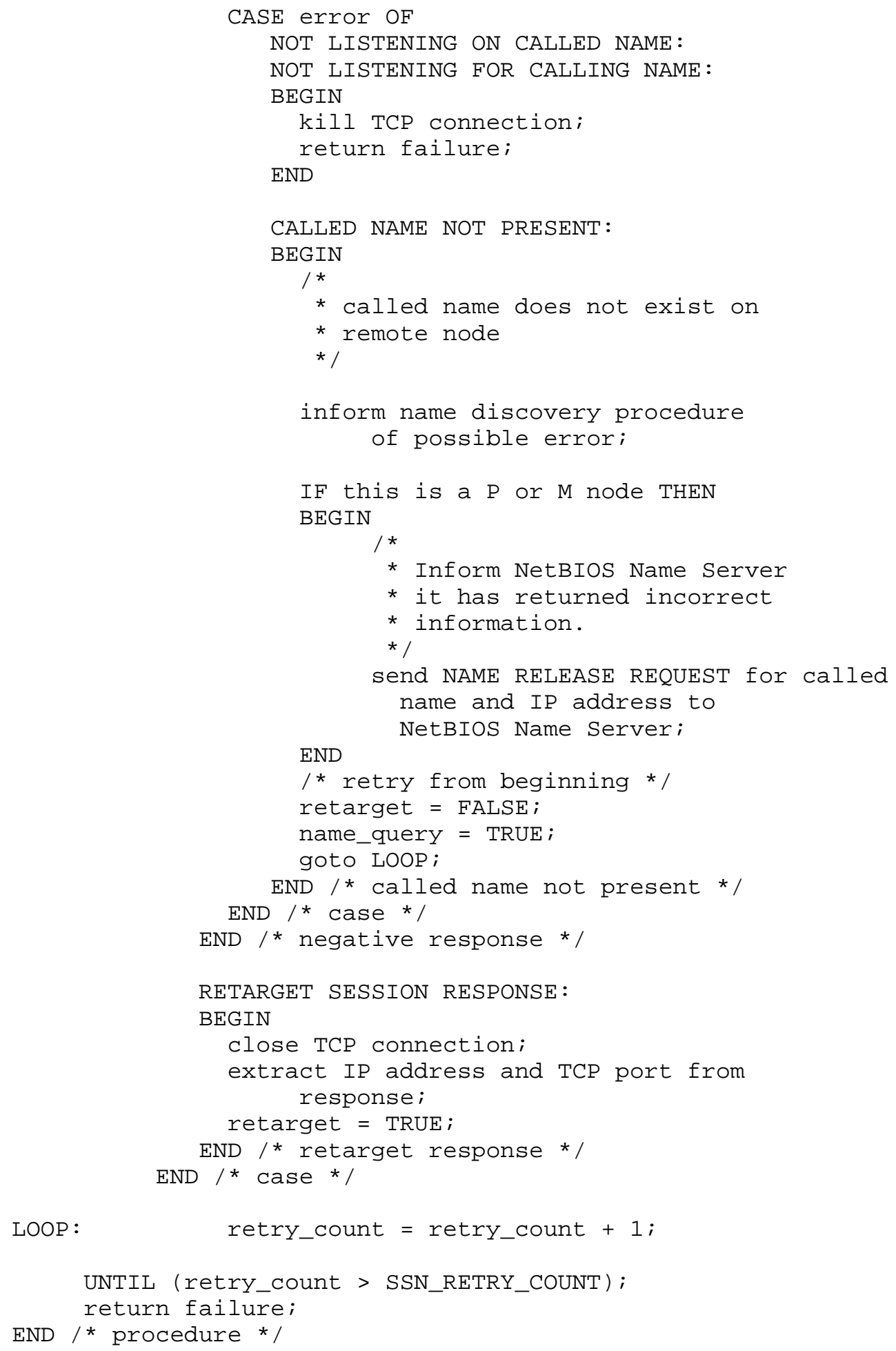




\subsubsection{RECEIVED PACKET PROCESSING}

These are packets received on a TCP connection before a session has been established. The listen routines attached to a NetBIOS user process need not implement the RETARGET response section. The user process version, separate from a shared Session Service, need only accept (POSITIVE SESSION RESPONSE) or reject (NEGATIVE SESSION RESPONSE) a session request.

PROCEDURE session_packet (packet)

/*

* processing initiated by receipt of a session service

* packet for a session in the session establishment phase.

* Assumes the TCP connection has been accepted.

* $/$

BEGIN

CASE packet type

SESSION REQUEST:

BEGIN

IF called name does not exist on node THEN

BEGIN

send NEGATIVE SESSION RESPONSE with CALLED

NAME NOT PRESENT error code;

close TCP connection;

END

Search for a listen with CALLING NAME for CALLED NAME;

IF matching listen is found THEN

BEGIN

IF port of listener process is port TCP connection is on THEN

BEGIN

send POSITIVE SESSION RESPONSE;

Hand off connection to client process and/or inform user session is established;

END

ELSE

BEGIN

send RETARGET SESSION RESPONSE with listener's IP address and TCP port;

close TCP connection;

END

END

ELSE

BEGIN

/* no matching listen pending */ 


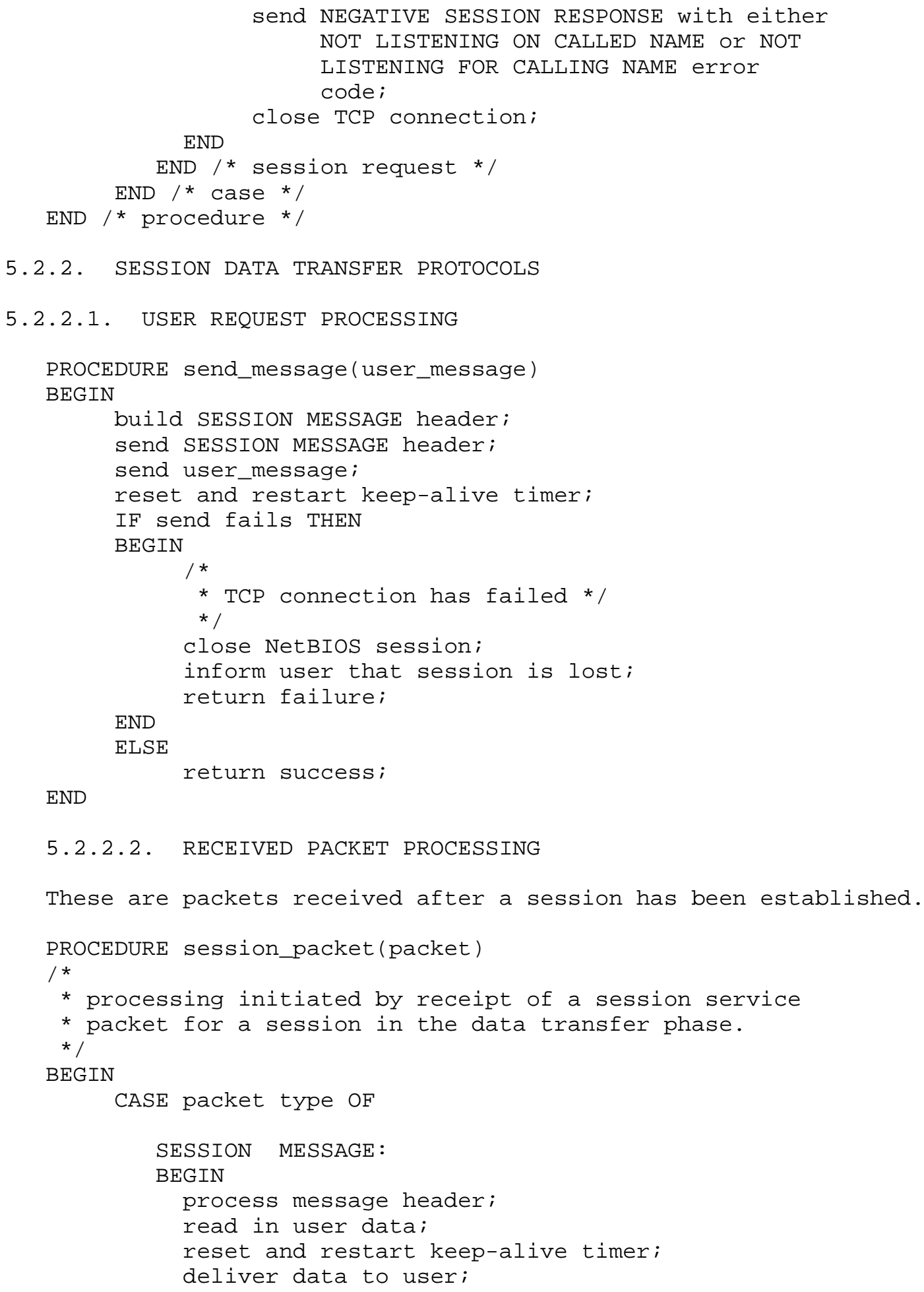

\subsubsection{RECEIVED PACKET PROCESSING}

These are packets received after a session has been established. 


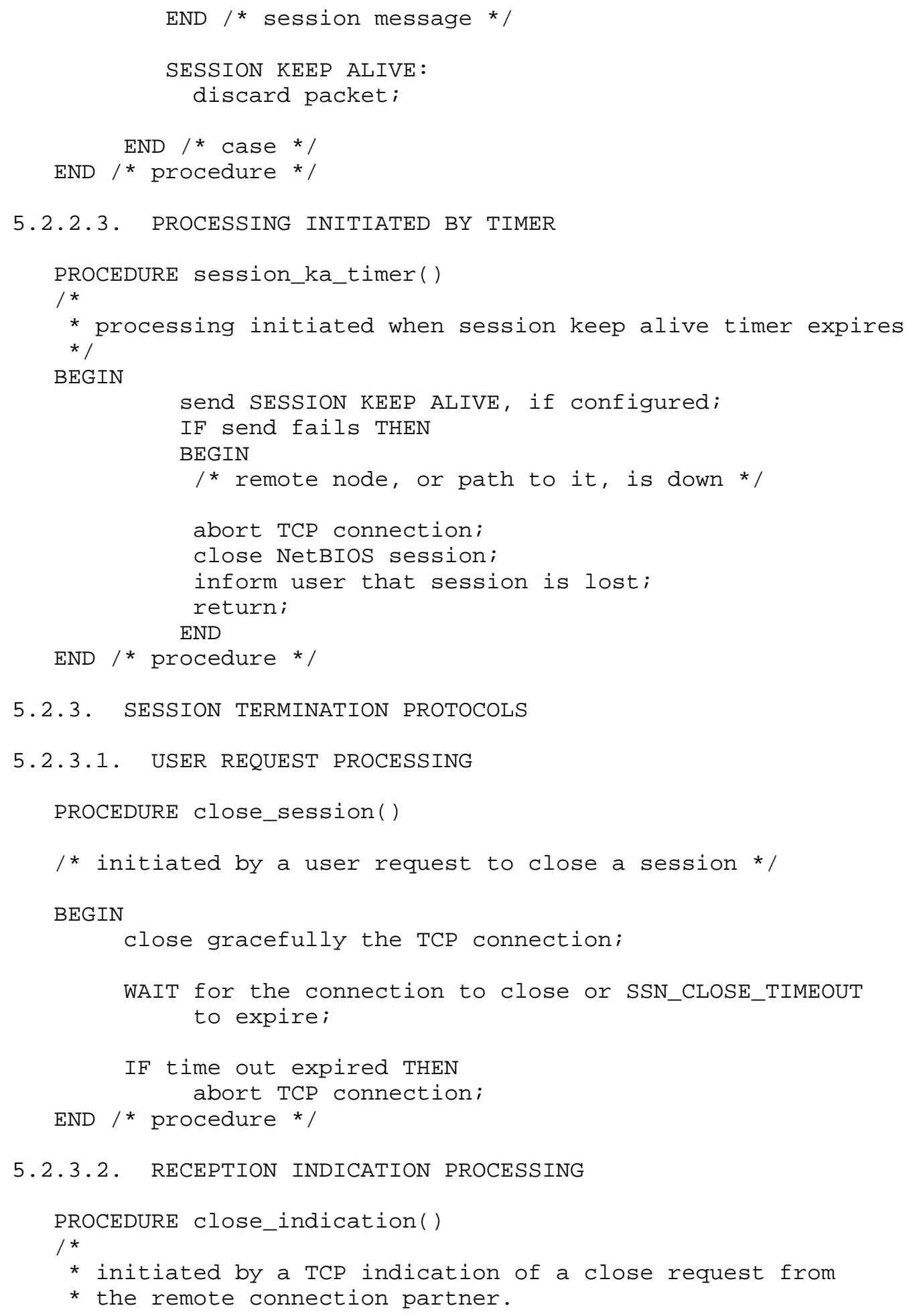




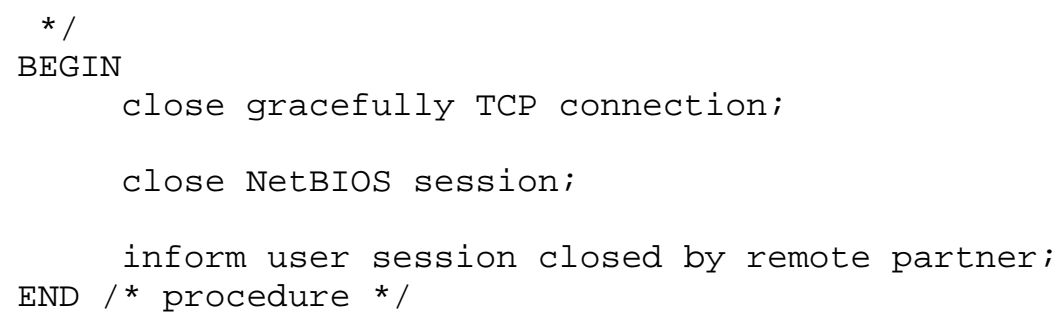

5.3. NetBIOS DATAGRAM SERVICE PROTOCOLS

The following are GLOBAL variables and should be NetBIOS user configurable:

- SCOPE_ID: the non-leaf section of the domain name preceded by a '.' which represents the domain of the NetBIOS scope for the NetBIOS name. The following protocol description only supports single scope operation.

- MAX_DATAGRAM_LENGTH: the maximum length of an IP datagram. The minimal maximum length defined in for IP is 576 bytes. This value is used when determining whether to fragment a NetBIOS datagram. Implementations are expected to be capable of receiving unfragmented NetBIOS datagrams up to their maximum size.

- BROADCAST_ADDRESS: the IP address B-nodes use to send datagrams with group name destinations and broadcast datagrams. The default is the IP broadcast address for a single IP network.

The following are Defined Constants for the NetBIOS Datagram Service:

- DGM_SRVC_UDP_PORT: the globally well-known UDP port allocated where the NetBIOS Datagram Service receives UDP packets. See section 6, "Defined Constants", for its value.

\subsubsection{B NODE TRANSMISSION OF NetBIOS DATAGRAMS}

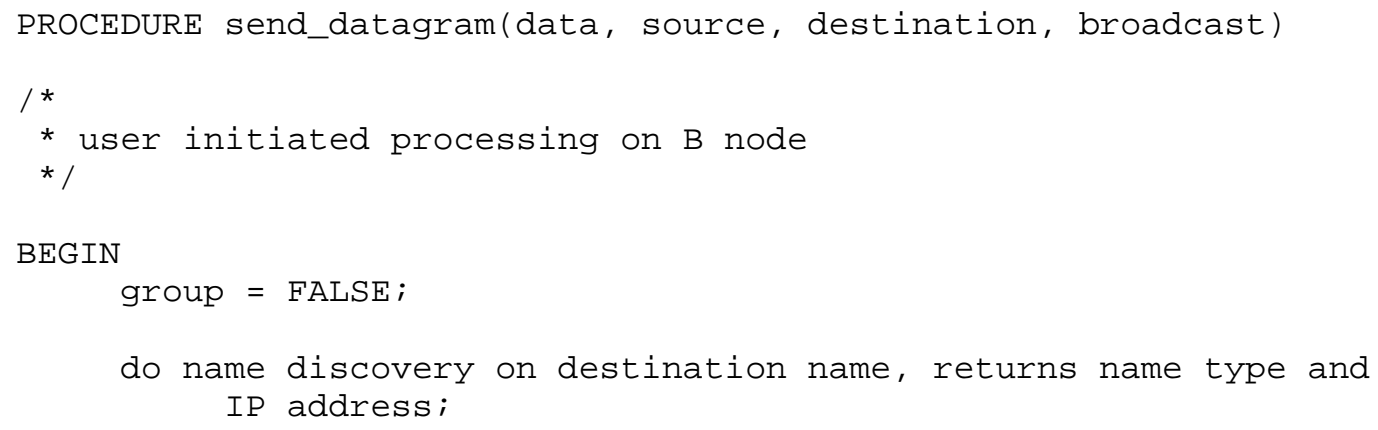




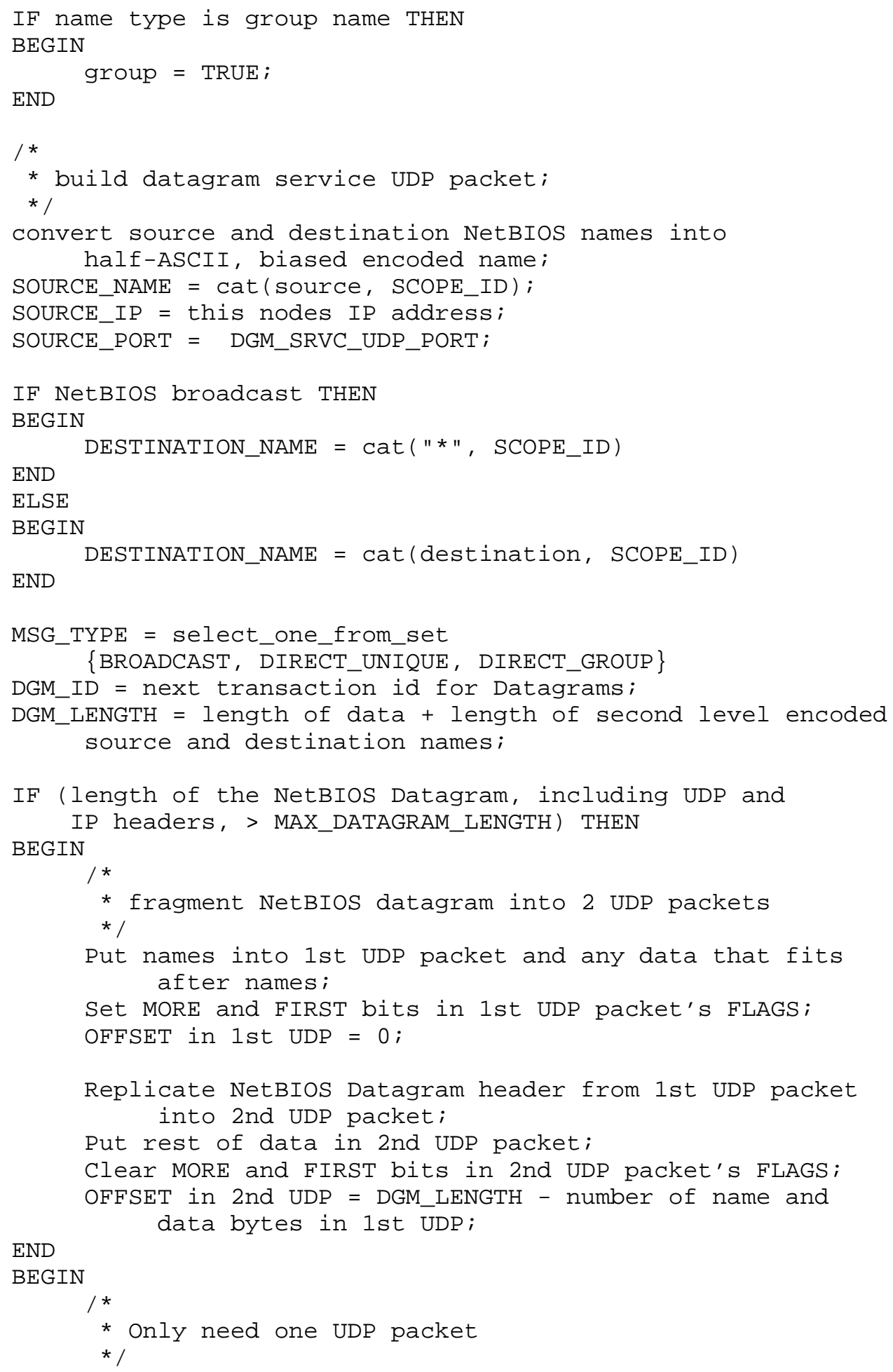




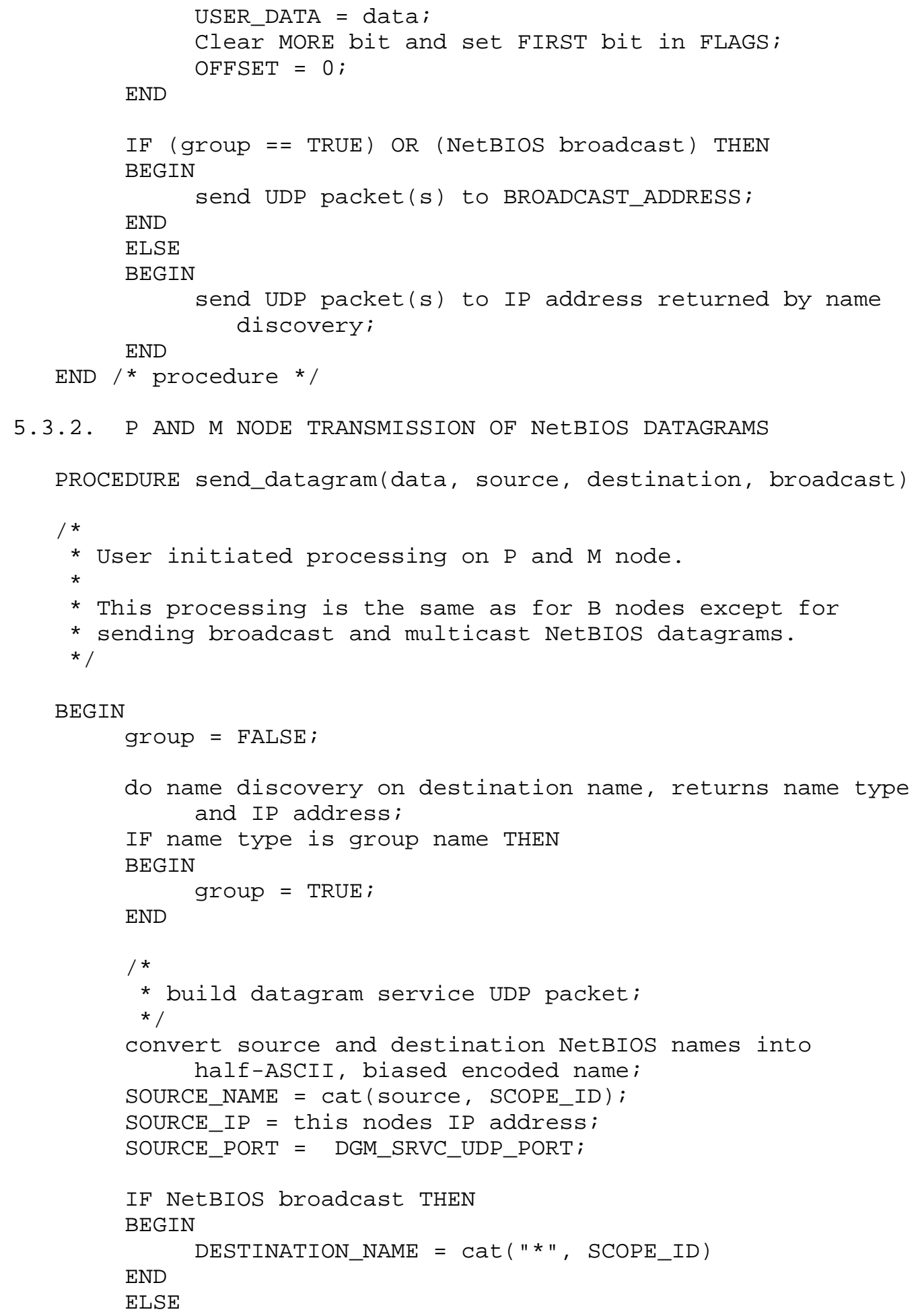




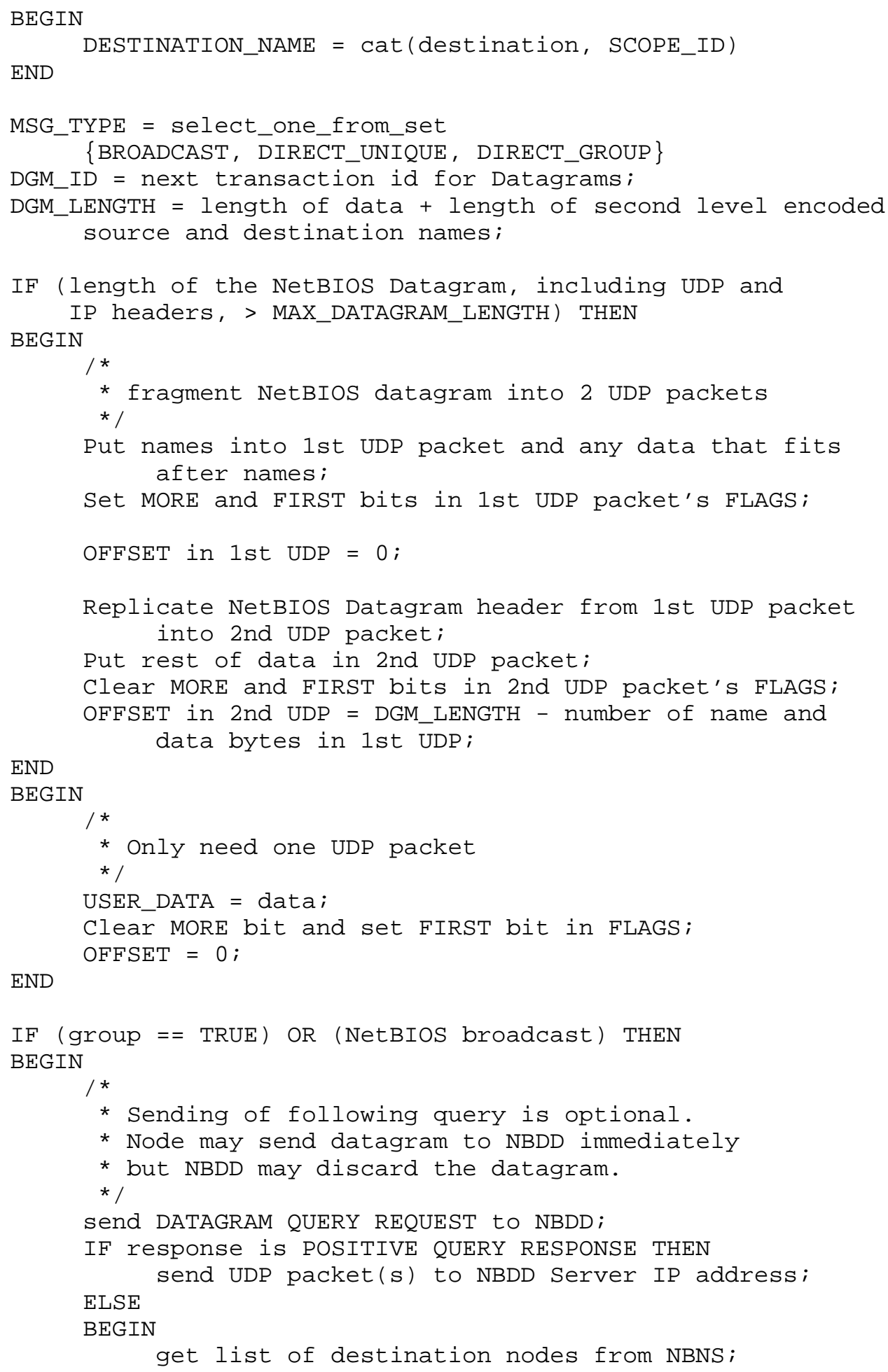




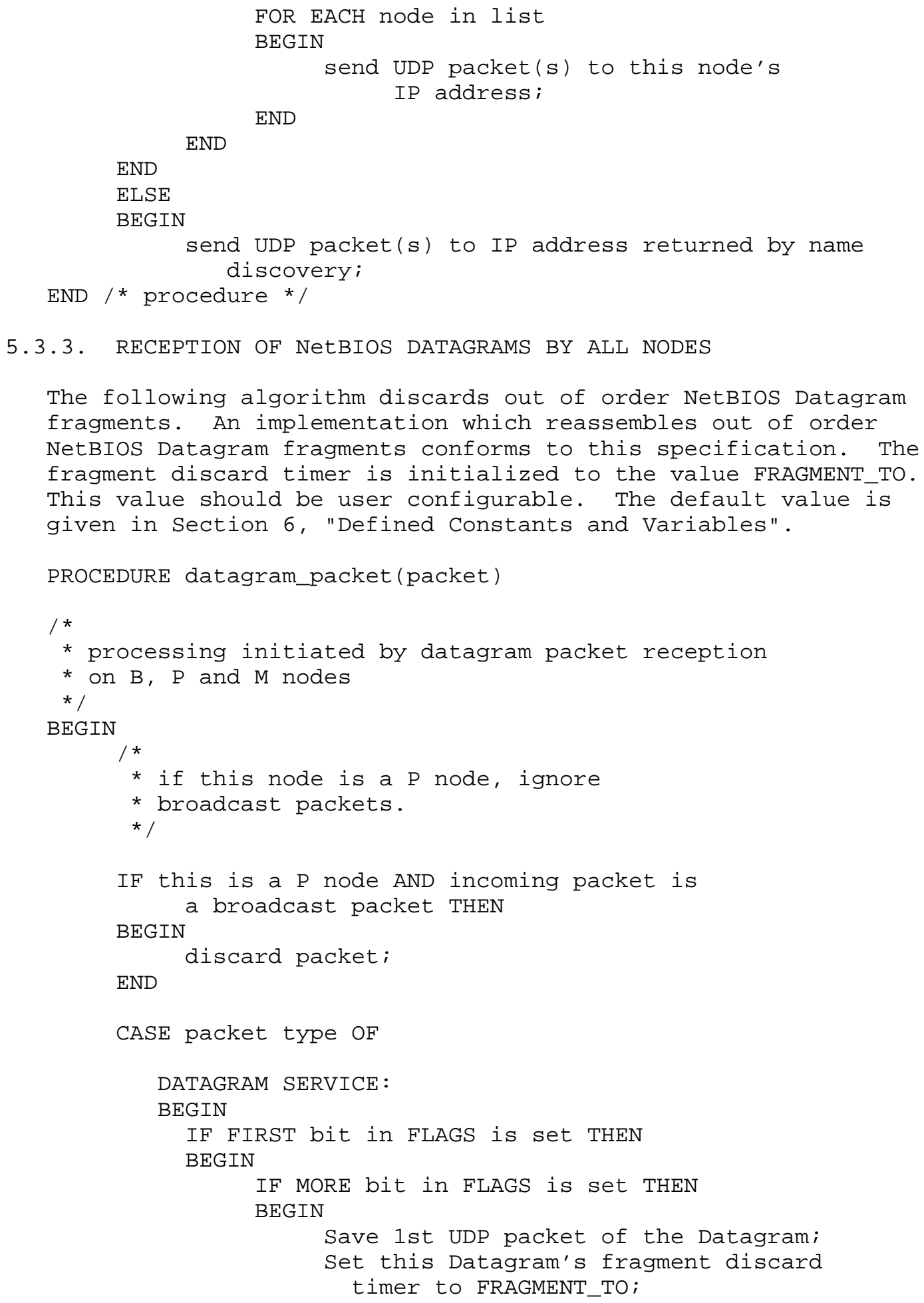




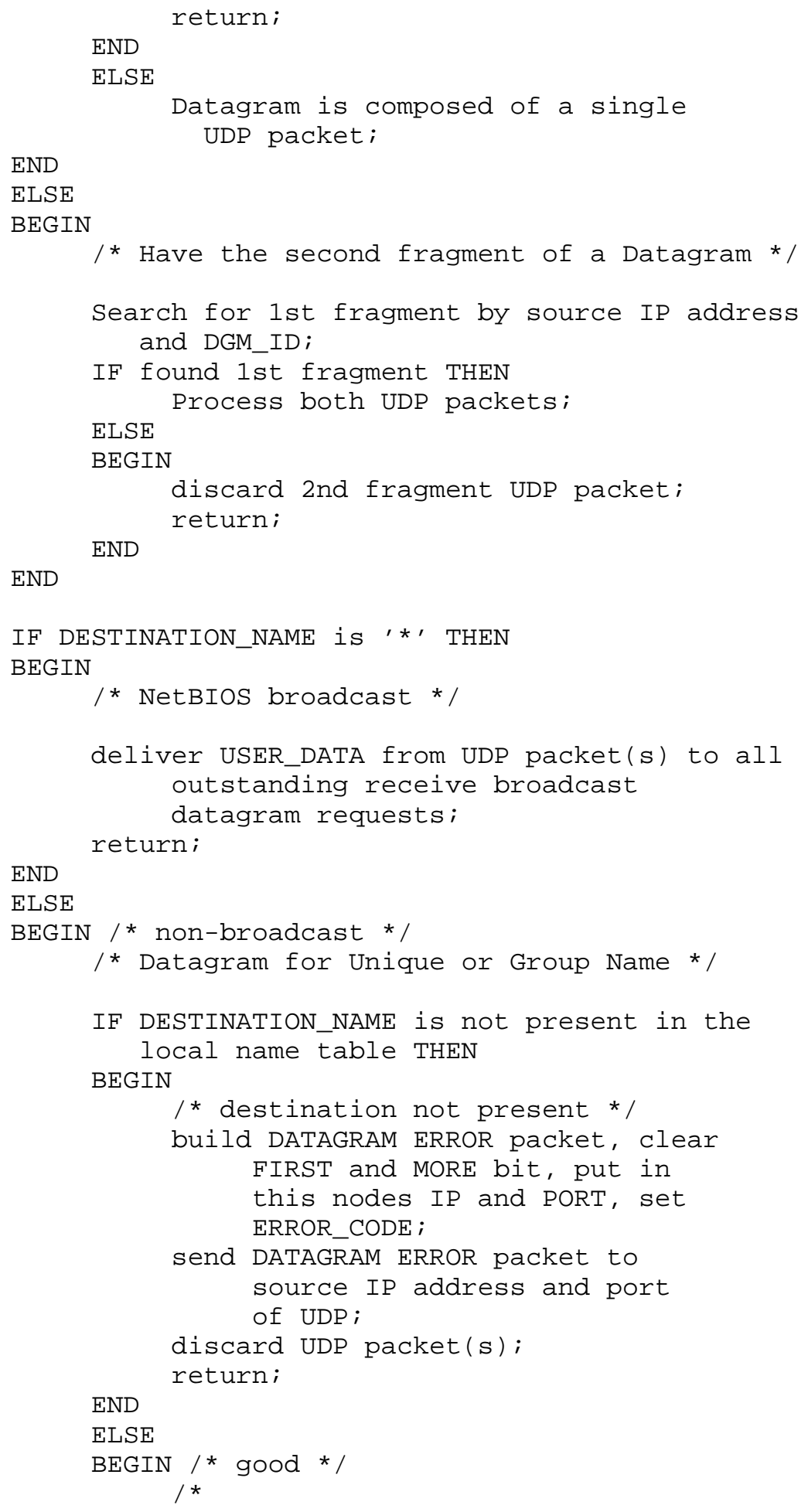




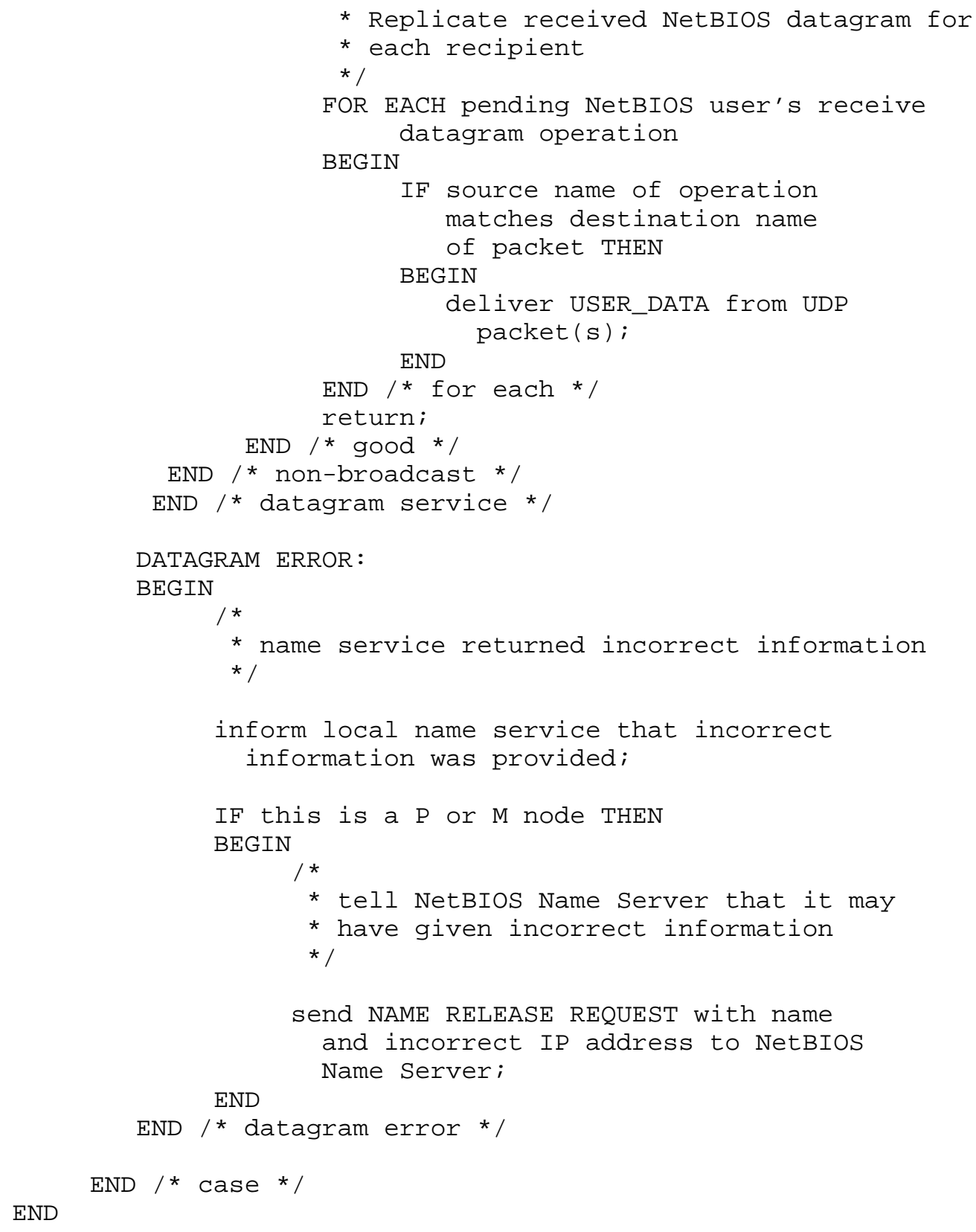




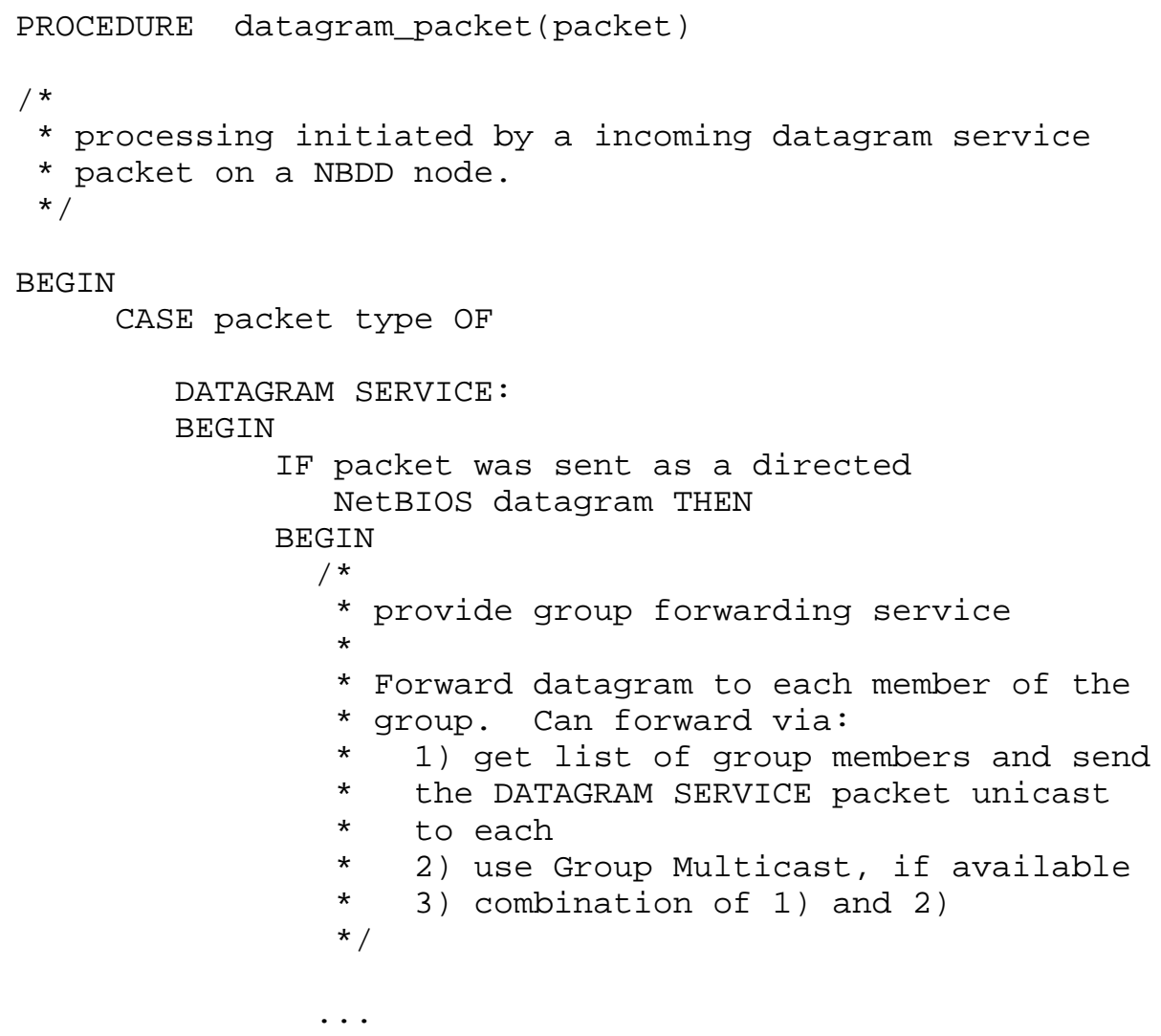




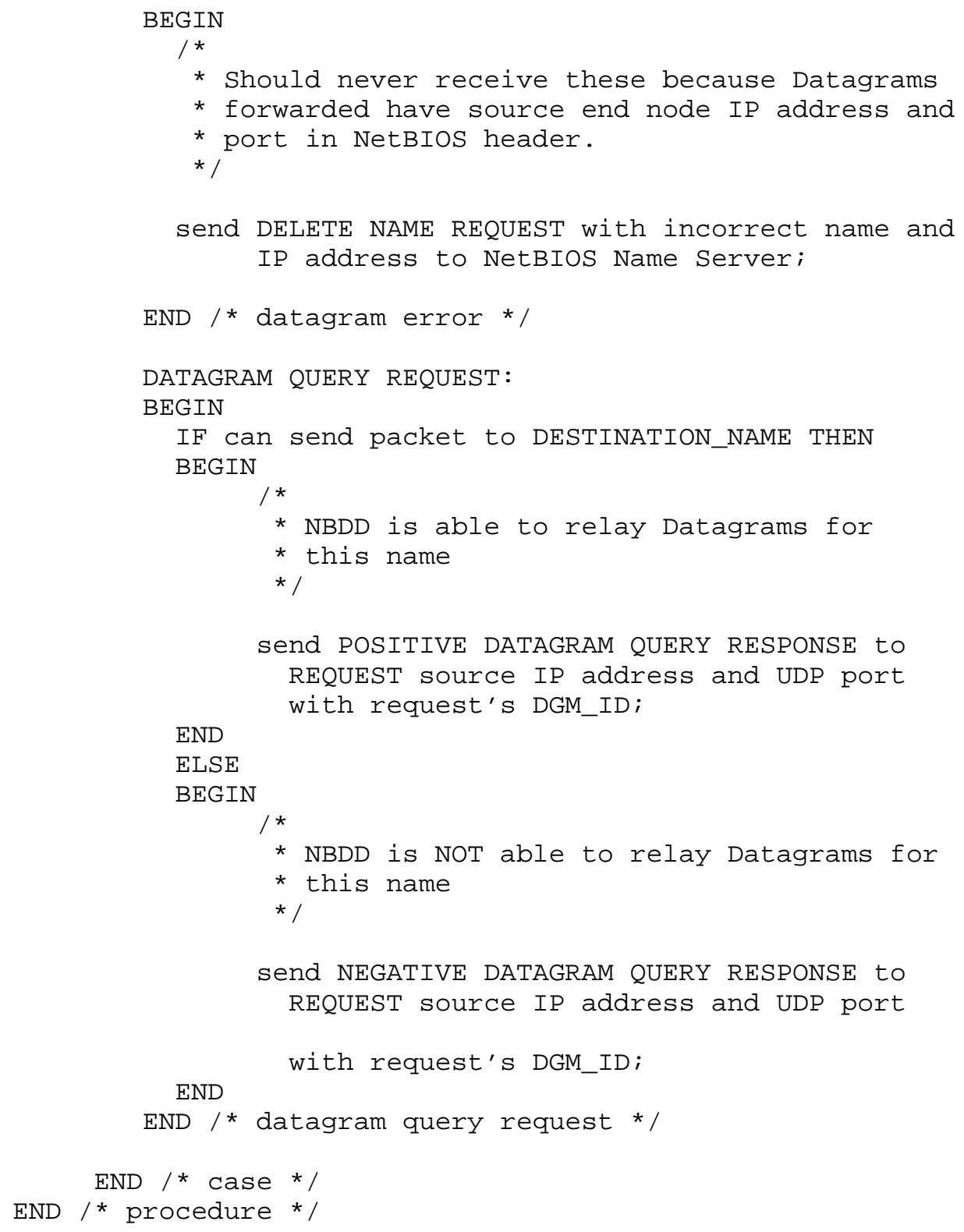


6. DEFINED CONSTANTS AND VARIABLES

GENERAL :

SCOPE_ID

BROADCAST_ADDRESS

BCAST_REQ_RETRY_TIMEOUT

BCAST_REQ_RETRY_COUNT

UCAST_REQ_RETRY_TIMEOUT

UCAST_REQ_RETRY_COUNT

MAX_DATAGRAM_LENGTH

NAME SERVICE :

REFRESH_TIMER

CONFLICT_TIMER

NAME_SERVICE_TCP_PORT
The name of the NetBIos scope.

This is expressed as a character string meeting the requirements of the domain name system and without a leading or trailing "dot".

An implementation may elect to make this a single global value for the node or allow it to be specified with each separate NetBIOS name (thus permitting cross-scope references.)

An IP address composed of the nodes's network and subnetwork numbers with all remaining bits set to one.

I.e. "Specific subnet" broadcast addressing according to section 2.3 of RFC 950 .

250 milliseconds. An adaptive timer may be used.

3

5 seconds An adaptive timer may be used. 3 576 bytes (default)
Negotiated with NBNS for each name.

1 second Implementations may chose a longer value.

137 (decimal) 


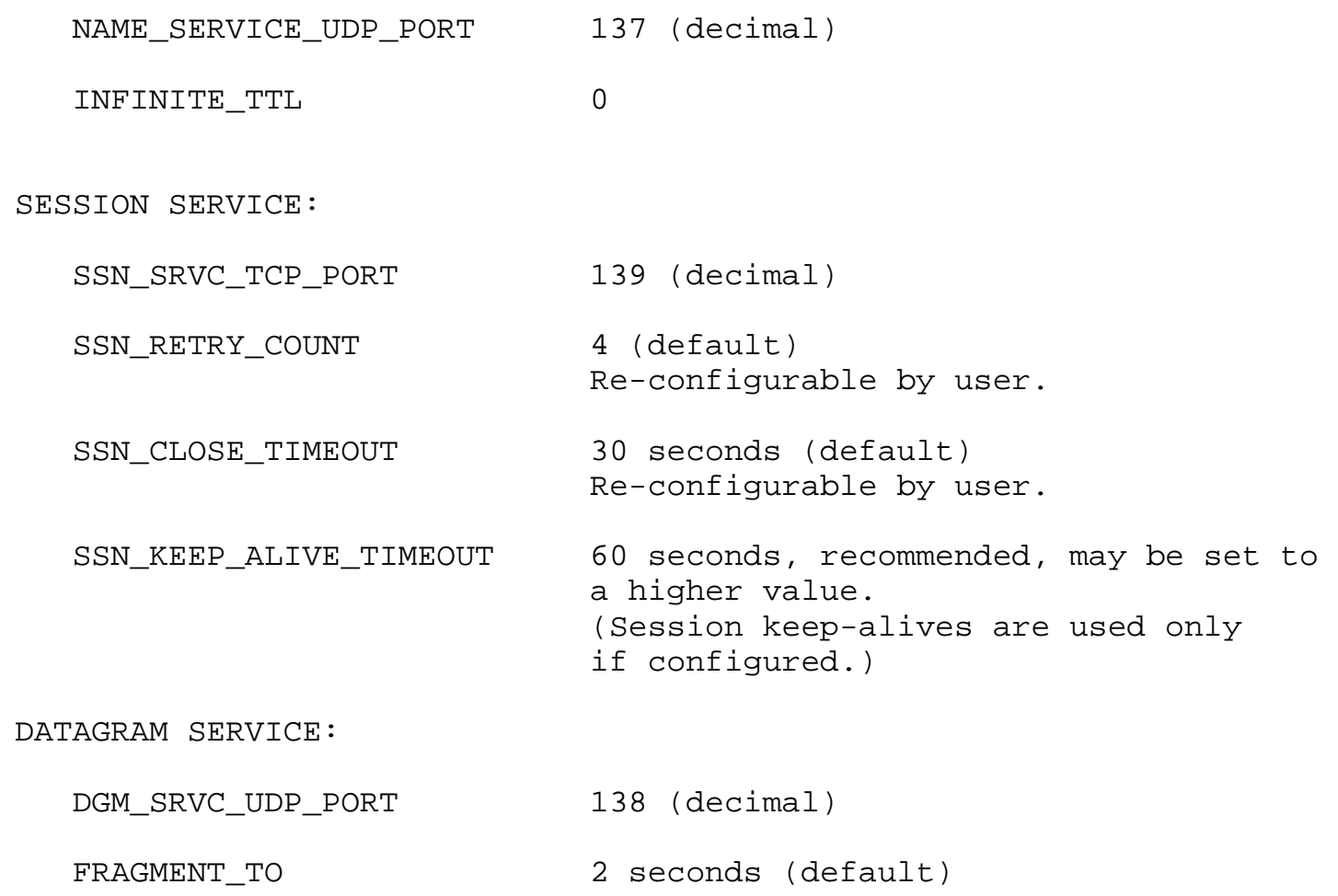




\section{REFERENCES}

[1] "Protocol Standard For a NetBIOS Service on a TCP/UDP Transport: Concepts and Methods", RFC 1001, March 1987.

[2] J. Reynolds, J. Postel, "Assigned Numbers", RFC 990, November 1986.

[3] P. Mockapetris, "Domain Names - Implementation and Specification", RFC 883, November 1983. 